\title{
KERNELS FOR THE TANGENTIAL CAUCHY-RIEMANN EQUATIONS
}

\author{
AL BOGGESS
}

\begin{abstract}
On certain codimension one and codimension two submanifolds in $\mathbf{C}^{n}$, we can solve the tangential Cauchy-Riemann equations $\bar{\partial}_{b} u=f$ with an explicit integral formula for the solution.

Let $M=\partial D$, where $D$ is a strictly pseudoconvex domain in $C^{n}$. Let $\omega \subset \subset M$ be defined by $\omega=\{z \in M ; \operatorname{Re} h(z)<0\}$, where $h$ is holomorphic near $\bar{D}$. Points on the boundary of $\omega, \partial \omega$, where the tangent space of $\partial \omega$ becomes complex linear, are called characteristic points.

TheOREM 1. Suppose $\partial \omega$ is admissible (in particular if $\partial \omega$ has two characteristic points). Suppose $f \in \mathcal{E}_{M}^{p, q}(\bar{\omega}), 1<q<n-3$, is smooth on $\omega$ and satisfies $\bar{\partial}_{M} f=0$ on $\omega$; then there exists $u \in \mathcal{E}_{M}^{p-1}(\omega)$ which is smooth on $\bar{\omega}$ except possibly at the characteristic points on $\partial \omega$ and which solves the equation $\bar{\partial}_{M} u=f$ on $\omega$.

THEOREM 2. Suppose $f \in \mathcal{E}_{M}^{p q}(\omega), 2<q<n-3$, is smooth on $\omega$; vanishes near each characteristic point; and $\bar{\partial}_{M} f=0$ on $\omega$. Then there exists $u \in \mathcal{E}_{M}^{p-1}(\omega)$ satisfying $\bar{\partial}_{M} u=f$ on $\omega$.

THEOREM 3. Suppose $f \in \mathscr{D}_{M}^{g} q(\omega), 2<q<n-3$, is smooth with compact support in $\omega$, and $\bar{\partial}_{M} f=0$. Then there exists $u \in \mathscr{D P}_{M}^{q^{-1}}(\omega)$ with compact support in $\omega$ and which solves $\bar{\partial}_{M} u=f$.

In all three theorems we have an explicit integral formula for the solution.

Now suppose $S=\partial \omega$. Let $C_{S}$ be the set of characteristic points on $S$. We construct an explicit operator $E: \mathscr{D}_{S}^{p, q}\left(S-C_{S}\right) \rightarrow \mathcal{E}_{S}^{p, q-1}\left(S-C_{S}\right)$ with the following properties.

THEOREM 4. The operator $E$ maps $L_{p, \text { comp }}^{*}\left(S-C_{S}\right) \rightarrow L_{p, \text { loc }}^{*}\left(S-C_{S}\right)$ and if $f \in$ की $p_{S}^{p, q}\left(S-C_{S}\right), 1<q<n-3$, then $f=\bar{\partial}_{S}\{E(f)\}+E\left(\bar{\partial}_{S} f\right)$.
\end{abstract}

\section{Chapter 1. Introduction}

There has been a substantial amount of recent literature on the kernel approach to solve the Cauchy-Riemann equations on $\mathbf{C}^{n}$ and the tangential Cauchy-Riemann equations on a hypersurface in $\mathbf{C}^{\boldsymbol{n}}$. For example, Romanov [16] discovered a kernel (which we call $R$ ) that globally solves the tangential Cauchy-Riemann equations on a strictly pseudoconvex hypersurface. The purpose of this work is to use kernels to solve three local theorems concerning the tangential Cauchy-Riemann equations on a strictly pseudoconvex hypersurface and to solve a global theorem concerning the tangential Cauchy-Riemann equations on certain codimension two submanifolds in $\mathbf{C}^{n}$.

Specifically, let $M=\partial D$, where $D$ is a strictly pseudoconvex domain in $\mathbf{C}^{n}$. Let $\omega \subset \subset M$ be an open subset defined by $\omega=\{z \in M ; \operatorname{Re} h(z)<0\}$ where $h$ is holomorphic near $\bar{D}$. The points in the boundary of $\omega(\partial \omega)$ where the tangent space

Received by the editors July 9, 1979; presented to the Society, April 28, 1979.

1980 Mathematics Subject Classification. Primary 32F20. 
of $\partial \omega$ becomes complex linear are called characteristic points. If $f$ is a smooth form on $\omega$ with $\bar{\partial}_{M} f=0$, then we wish to solve the equation $\bar{\partial}_{M} u=f$ on $\omega$. We prove three theorems about this equation. The first theorem assumes that $f$ is smooth on $\bar{\omega}$ except possibly at the characteristic points, and gets a solution which is smooth on $\bar{\omega}$ except possibly at the characteristic points. The second theorem assumes that $f$ is smooth on $\omega$ and vanishes near each characteristic point on $\partial \omega$, and gets a solution which is smooth on $\omega$. The interesting thing here is that nothing is assumed about the growth of $f$ near $\partial \omega$ away from the characteristic points. The third theorem assumes that $f$ has compact support in $\omega$ and gets a solution with compact support in $\omega$. In all three theorems, certain endpoint bidegrees must be avoided. The characteristic points on $\partial \omega$ must be avoided because $\partial \omega$ becomes characteristic to $\bar{\partial}_{M}$ at these points. The solution in each of our three theorems can be represented by a kernel. These kernels were introduced by Henkin and Romanov (although they were described much differently by them). Our point of view here is to take principal value limits across the singular sets of these kernels. This point of view is used by Harvey and Polking [6] to solve the $\bar{\partial}$ equation on open subsets in $\mathbf{C}^{\boldsymbol{n}}$. In fact, much of our work is motivated by their work on $\mathbf{C}^{\boldsymbol{n}}$.

Next, we study the tangential Cauchy-Riemann equations on a class of codimension two submanifolds. Let $S=\partial \omega$. We construct an explicit map $E$ : $\mathscr{Q}_{S}^{p, q} \rightarrow$ $\mathcal{E}_{S}^{p, q-1}$ which is also continuous in the $\mathcal{L}^{p}$ norm and such that if $f \in \mathscr{D}_{S}^{p, q}$, then $f=\bar{\partial}_{S}[E(f)]+E\left(\bar{\partial}_{S} f\right)$. Again, we must avoid the characteristic points in $S$. The operator $E$ is selfadjoint up to sign, and is the boundary value jump across $S$ of one of the kernels used to solve the local $\bar{\partial}_{M}$ theorems mentioned above. To construct the operator $E$, we make use of the boundary value jump across $S$ of

$$
\int_{\zeta \in S} R(\zeta, z) \wedge f(\zeta)
$$

We prove that $f=R^{+} f-R^{-} f$ away from characteristic points, where $R^{ \pm} f$ denotes the boundary values of (1.1) on $S$ from either side. A consequence which is of independent interest is the result that any $\bar{\partial}_{S}$ closed form is the boundary value jump of a $\bar{\partial}_{M}$ closed form on $M-S$. Both local and global results are obtained.

We have organized our work as follows. In Chapter 2, we discuss the kernels we use and some formal identities which relate them to the $\bar{\partial}_{M}$ operator. In Chapter 3, we review the basic facts we need concerning the global solution to $\bar{\partial}_{M}$ (the $R$ kernel). Chapter 4 contains our result on principal value limits and the three theorems on the local solution to $\bar{\partial}_{M}$. In Chapter 5 , we construct the global solution to $\bar{\partial}_{S}$. The technical details in Chapters 4 and 5 are given at the end of each chapter so as not to interrupt the flow of the basic argument.

\section{Chapter 2. Preliminaries}

2.1. Notation. Throughout this work $\Lambda^{p, q}\left(\mathbf{C}^{n}\right)$ will denote the bundle of forms in $\mathbf{C}^{n}$ of bidegree $p, q$. We denote the projection maps by

$$
\pi^{p, q}: \Lambda^{p+q}\left(\mathbf{C}^{n}\right) \rightarrow \Lambda^{p, q}\left(\mathbf{C}^{n}\right) \text {. }
$$

$\mathcal{E}^{p, q}\left(\mathbf{C}^{n}\right)$ will be the space of all smooth sections of $\Lambda^{p, q}\left(\mathbf{C}^{n}\right)$ and $\mathscr{D}^{p, q}\left(\mathbf{C}^{n}\right)$ will be the space of those sections of $\mathcal{E}^{p, q}\left(C^{n}\right)$ with compact support. In the dual setting, 
we have currents of bidegree $p, q$ denoted by $\mathscr{D}^{\prime p, q}\left(\mathbf{C}^{n}\right)$ and currents of type $p, q$ with compact support, denoted by $\mathcal{E}^{\prime p, q}\left(\mathbf{C}^{n}\right)$. The current pairing in $\mathbf{C}^{n}$ will be denoted by $(T, u)_{\mathbf{C}^{n}}$ where $T \in \mathcal{E}^{\prime p, q}\left(\mathbf{C}^{n}\right)$ and $u \in \mathcal{E}^{n-p, n-q}\left(\mathbf{C}^{n}\right)$. All spaces defined above will have their usual topologies.

Suppose $N$ is an oriented smooth submanifold of $\mathbf{C}^{n}$ of real dimension $2 n-k$ defined locally by $N=\left\{x \in \mathbf{C}^{n} ; \rho_{1}(x)=\cdots=\rho_{k}(x)=0\right\}$ where $\rho_{1}, \ldots, \rho_{k}$ are smooth real valued functions with $d_{\rho_{1}}(x) \wedge \cdots \wedge d_{\rho_{k}}(x) \neq 0$ for $x \in N$. The current 'integration over $N$,' denoted by $[N]$, is defined by $[N](\phi)=\int_{N} \phi$ where $\phi \in \mathscr{D}^{2 n-k}\left(\mathbf{C}^{n}\right) .[N]$ is a current of degree $k$ and can be written locally as

$$
[N]=g \sigma_{N} d_{\rho_{1}} \wedge \cdots \wedge d_{\rho_{k}}
$$

where $\sigma_{N}$ is surface measure on $N$, and $g$ is a nonzero smooth function. The current $[N]$ splits into various bidegrees, i.e. $[N]=\sum_{p+q=k}[N]^{p, q}$ where $[N]^{p, q}$ is the piece of $[N]$ of bidegree $p, q$. The most important piece of this splitting is $[N]^{0, k}$, which can be written locally as

$$
[N]^{0, k}=g \sigma_{N} \bar{\partial} \rho_{1} \wedge \cdots \wedge \bar{\partial} \rho_{k} .
$$

We denote the restriction of the bundle $\Lambda^{p, q}\left(\mathbf{C}^{n}\right)$ to $N$ by $\left.\Lambda^{p, q}\left(\mathbf{C}^{n}\right)\right|_{N}$. A section of $\left.\Lambda^{p, q}\left(\mathbf{C}^{n}\right)\right|_{N}$ is obtained by restricting the coefficients of an ambient $p, q$ form to $N$. We let $C_{N}$ be the closed set in $N$ such that $N-C_{N}$ is the open set where the tangent bundle of $N$ is minimally complex. Locally, we have

$$
C_{N}=\left\{x \in N ; \bar{\partial} \rho_{1}(x) \wedge \cdots \wedge \bar{\partial} \rho_{k}(x)=0\right\} .
$$

We call $C_{N}$ the set of characteristic points of $N$. Over the open set $N-C_{N}$, we let $\Lambda_{N}^{p, q}$ denote the bundle of $p, q$ forms which are complex tangential to $N$. Locally, $\Lambda_{N}^{p, q}$ consists of those sections in $\left.\Lambda^{p, q}\left(\mathbf{C}^{n}\right)\right|_{N}$ which are orthogonal (Euclidean metric) to the ideal in $\left.\Lambda^{*}\left(C^{n}\right)\right|_{N}$ generated by $\bar{\partial} \rho_{1}, \ldots, \bar{\partial} \rho_{k}$. We let $t_{N}$ : $\left.\Lambda^{p, q}\left(\mathbf{C}^{n}\right)\right|_{N-C_{N}} \rightarrow \Lambda_{N}^{p, q}$ be the orthogonal projection onto $\Lambda_{N}^{p, q}$. If $f \in \Lambda^{p, q}\left(\mathbf{C}^{n}\right)$, then we of ten write $f_{t_{N}}$ for $t_{N}(f)$ and we call $f_{t_{N}}$ the complex tangential piece of $f$ along $N$.

If $V \subset N-C_{N}$ is an open set, then $\mathcal{E}_{N}^{p, q}(V)$ will denote the space of smooth sections of $\Lambda_{N}^{p, q}$ over $V$, and $\mathscr{D}_{N}^{p, q}(V)$ will be those sections of $\mathcal{E}_{N}^{p, q}(V)$ with compact support in $V$. In the dual setting, $\mathscr{D}_{N}^{\prime p, q}(V)$ will be the space of currents on $V$ of bidegree $p, q$ which are complex tangential to $N$, and $\mathcal{E}_{N}^{\prime p, q},(V)$ will be those currents in $\mathscr{D}_{N}^{\prime p, q}(V)$ with compact support in $V$. The current pairing on $N$ will be denoted by $(T, u)_{N}$ and is defined as follows: If $T \in \mathcal{E}_{N}^{p, q}(V)$ and $u \in$ $\mathscr{D}_{N}^{n-p, n-q-k}(V)$ are smooth forms, then

$$
(T, u)_{N}=\left([N]^{0, k} \wedge \tilde{T}, \tilde{u}\right)_{\mathrm{C}^{n}}=\int_{N} \tilde{T} \wedge \tilde{u}
$$

where $\tilde{T}$ and $\tilde{u}$ are ambient extensions of $T$ and $u$, respectively. This definition naturally extends to $T \in \mathscr{D}_{N}^{\prime p, q}(V)$ provided we have a good definition of $[N]^{0, k} \wedge$ $T$ (cf. Harvey and Polking, Remark 7.6). The map $t_{N}: \mathbb{Q}^{n-p, n-q-k}\left(\mathbf{C}^{n}\right) \rightarrow$ $\mathscr{D}_{N}^{n-p, n-q-k}(V)$ has (current) adjoint $t_{N}^{\prime}: \mathscr{D}_{N}^{\prime p, q}(V) \rightarrow \mathscr{D}_{N}^{\prime p, q+k}\left(\mathbf{C}^{n}\right)$ where $\mathscr{D}_{N}^{\prime}\left(\mathbf{C}^{n}\right)$ is the space of currents on $\mathbf{C}^{n}$ with support in $N$. If $T \in \mathscr{Q}_{N}^{p, q}(V)$ is a smooth form, 
then

$$
t_{N}^{\prime}(T)=[N]^{0, k} \wedge T
$$

This is because

$$
\begin{aligned}
\left(t_{N}^{\prime}(T), \phi\right)_{\mathbf{C}^{n}}=\left(T, \phi_{t_{N}}\right)_{N}=\int_{N} T \wedge \phi_{t_{N}}=\left([N]^{0, k} \wedge T, \phi\right)_{\mathbf{C}^{n}} & \\
& \text { for } \phi \in \mathscr{Q})^{n-p, n-q-k}\left(\mathbf{C}^{n}\right) .
\end{aligned}
$$

Therefore if $T \in \mathscr{D}_{N}^{\prime p, q}(V)$, then it is natural to define $[N]^{0, k} \wedge T=t_{N}^{\prime}(T)$.

Suppose $S \subset N$ is a hypersurface in $N$. The current 'integration over $S$ ' can be regarded either as a current on $\mathbf{C}^{n}$ of degree $k+1$, or as a current on $N$ of degree one. If we wish to think of $S$ as a current on $N$, we shall denote it by $[S]^{0,1}$. If $f$ is a smooth form on $S$, then we have

$$
\left([S]^{0,1}, f\right)_{N}=\left([S]^{0, k+1}, \tilde{f}\right)_{\mathbf{C}^{n}}=\int_{S} \tilde{f}
$$

where $\tilde{f}$ is any ambient extension of $f$.

The induced Cauchy-Riemann operator $\bar{\partial}_{N}: \mathcal{E}_{N}^{p, q} \rightarrow \mathcal{E}_{N}^{p, q+1}$ can now be defined as follows: Suppose $V \subset N-C_{N}$ is an open set and suppose $f \in \mathcal{E}_{N}^{p, q}(V)$. Choose $\tilde{f} \in \mathcal{E}^{p, q}\left(\mathbf{C}^{n}\right)$ with $\tilde{f}_{t_{N}}=f$. Then

$$
\bar{\partial}_{N} f=(\bar{\partial} \tilde{f})_{t_{N}} \in \mathcal{E}_{N}^{p, q+1}(V) .
$$

It is easy to show the definition of $\tilde{\partial}_{N} f$ is independent of the extension $\tilde{f}$. Note that $\bar{\partial}_{N} f(x)$ is not defined for $x \in C_{N}$ because $\tilde{f}_{t_{N}}(x)$ is not defined for $x \in C_{N}$. By taking an extension $\tilde{f}$ which is independent of the normal directions of $N$, one can see that the vector fields occurring in $\bar{\partial}_{N}$ must be tangential to $N$.

2.1. Proposition. Suppose $V$ is an open subset of $N-C_{N}$ and $f \in \mathcal{E}_{N}^{p, q}(V)$. Then $\bar{\partial}_{N} f=h$ if and only if $\bar{\partial}\left\{\tilde{f} \wedge[N]^{0, k}\right\}=\tilde{h} \wedge[N]^{0, k}$ where $\tilde{f}$ and $\tilde{h}$ are smooth forms on $\mathbf{C}^{n}$ with $\tilde{f}_{t_{N}}=f$ and $\tilde{h}_{t_{N}}=h$.

Proof. By Stokes' theorem, we have $d[N]=0$. Therefore, by type considerations, it is clear that $\bar{\partial}[N]^{0, k}=0$. So, we have $\bar{\partial}\left\{\tilde{f} \wedge[N]^{0, k}\right\}=\bar{\partial} \tilde{f} \wedge[N]^{0, k}$. Since $[N]^{0, k}=\sigma_{N} g \bar{\partial} \rho_{1} \wedge \cdots \wedge \bar{\partial} \rho_{k}$, we see that

$$
\bar{\partial} \tilde{f} \wedge[N]^{0, k}=\tilde{h} \wedge[N]^{0, k}
$$

if and only if $(\bar{\partial} \tilde{f})_{t_{N}}=\tilde{h}_{t_{N}}$. This occurs if and only if $\bar{\partial}_{N} f=h$ by definition. Q.E.D.

Now we specialize to the case where $N=M$ is an oriented smooth hypersurface in $\mathbf{C}^{n}$ defined by $M=\left\{z \in \mathbf{C}^{n} ; \rho(z)=0\right\}$. We let $X$ be the vector field of type $(0,1)$ defined near $M$ which is dual to $\bar{\partial} \rho$. This means that

(2.2) $\left.X_{z}\right\lrcorner \bar{\partial} \rho(z)=1$ for $z$ near $M$, and

(2.3) $\left.X_{z}\right\lrcorner \psi(z)=0$ if and only if $\psi(z) \in \Lambda_{z}^{p, q}\left(\mathbf{C}^{n}\right)$ is orthogonal to the ideal in $\Lambda_{z}^{*}\left(\mathbf{C}^{n}\right)$ generated by $\bar{\partial} \rho(z)$. In particular $\left.X\right\lrcorner\left.\psi\right|_{M}=0$ if and only if $\left.\psi\right|_{M} \in \Lambda_{M}^{p, q}$.

Suppose $f \in \Lambda^{p, q}\left(\mathbf{C}^{n}\right)$; we define

$$
\begin{gathered}
\left.f_{T_{M}}=X\right\lrcorner(\bar{\partial} \rho \wedge f) \in \Lambda^{p, q}\left(\mathbf{C}^{n}\right), \\
\left.f_{N_{M}}=(X\lrcorner f\right) \in \Lambda^{p, q-1}\left(\mathbf{C}^{n}\right) .
\end{gathered}
$$


Since $\lrcorner$ is an antiderivation, we have:

$$
f(z)=f_{T_{M}}(z)+\bar{\partial} \rho(z) \wedge f_{N_{M}}(z) \text { for } z \text { near } M .
$$

It is easy to show that $\left.f_{T_{M}}\right|_{M}=f_{t_{M}}$ is the complex tangential piece of $f$ along $M$ (see Harvey and Polking [6]). We also define

$$
f_{n_{M}}=\left.f_{N_{M}}\right|_{M}
$$

and we call $f_{n_{M}}$ the complex normal piece of $f$ along $M$. Restricting equation (2.6) to $M$, we obtain

$$
f(z)=f_{t_{M}}(z)+\bar{\partial} \rho(z) \wedge f_{n_{M}}(z) \text { for } z \in M .
$$

Equation (2.8) provides an orthogonal decomposition of $f$ into its complex tangential and complex normal pieces. Keep in mind that $f_{T_{M}}$ and $f_{N_{M}}$ are defined near $M$, but $f_{t_{M}}$ and $f_{n_{M}}$ are defined only on $M$.

Now suppose that $S \subset M$ is an oriented, smooth hypersurface in $M$ defined by $S=\{z \in M ; r(z)=0\}$ where we assume $d_{\rho}(z) \wedge d r(z) \neq 0$ for $z \in S$. The set $C_{S}$ has the following characterization, which explains why we call $C_{s}$ the set of characteristic points.

2.9. Proposition. Let $z_{0} \in S$. The following are equivalent:

(a) $z_{0} \in C_{S}$.

(b) The tangent space of $S$ at $z_{0}, T_{z_{0}}(S)$, is complex linear.

(c) The symbol of $\bar{\partial}_{M}$ at $z_{0}$ applied to $(d r)_{t_{M}}, \sigma_{z_{0}}\left(\bar{\partial}_{M},(d r)_{t_{M}}\right)$ vanishes (i.e. $S$ is characteristic at $z_{0}$ for $\bar{\partial}_{M}$ ).

Proof. We note that $\sigma_{z_{0}}\left(\bar{\partial}_{M}, v\right)=\left(\pi^{0,1} v\right)_{t_{M}}$ for $v \in \Lambda_{z_{0}}^{1}(M)$. Therefore, $\sigma_{z_{0}}\left(\bar{\partial}_{M},(d r)_{t_{M}}\right)=0$ if and only if $(\bar{\partial} r)_{t_{M}}\left(z_{0}\right)=0$, i.e. if and only if $\bar{\partial}_{0} r\left(z_{0}\right)$ is a complex multiple of $\bar{\partial} \rho\left(z_{0}\right)$. Q.E.D.

2.10. EXAMPLE. Let $M$ be the unit sphere in $\mathbf{C}^{n}$ with defining function $\rho(z)=|z|$ - 1. Let $S=\left\{z \in M ; \operatorname{Re} z_{1}=0\right\}$ be the equator of the unit sphere. Then it is easy to see that $C_{S}=\{( \pm i, 0, \ldots, 0)\}$.

REMARK. A result of Wells [19] implies that if $S$ is a compact submanifold in $\mathbf{C}^{\boldsymbol{n}}$ with no characteristic points, then the Euler characteristic of $S$ is zero. Thus, if $S$ is homeomorphic to a $2 n-2$ real dimensional sphere, then the Euler characteristic of $S$ is two and so $C_{S}$ will be nonempty.

Away from $C_{S}$, we want to describe an explicit way of calculating the orthogonal projection:

$$
t_{s}:\left.\Lambda^{p, q}\left(\mathbf{C}^{n}\right)\right|_{s-c_{s}} \rightarrow \Lambda_{S}^{p, q} .
$$

It is clear from Proposition 2.9 that there is an open set $U$ in $\mathbf{C}^{n}$ containing $S-C_{S}$, such that $\bar{\partial} \rho(x) \wedge \bar{\partial} r(x) \neq 0$ for $x \in U$. Hence we can use Gram-Schmidt orthogonalization to choose a smooth form $\phi \in \mathcal{E}^{0,1}(U)$ with the following properties:

(2.11) $\langle\phi(x), \bar{\partial} \rho(x)\rangle=0$ for $x \in U$; or equivalently $X\lrcorner \phi=0$ on $U$. Here, $\langle$, denotes the Euclidean inner product on forms in $\mathbf{C}^{n}$.

(2.12) $|\phi(x)|=1$ for $x \in U$.

(2.13) There are smooth functions $\gamma(x) \neq 0$ and $\delta(x)$ on $U$ such that $\phi(x)=$ $\gamma(x) \bar{\partial} \rho(x)+\delta(x) \bar{\partial} r(x)$ for $x \in U$. 
Condition (2.13) implies that the ideal spanned by $\bar{\partial} \rho$ and $\bar{\partial} r$ is equal to the ideal spanned by $\bar{\partial} \rho$ and $\phi$. Hence, the definition of $\Lambda_{S}^{p, q}$ can be restated as those forms in $\left.\Lambda^{p, q}\left(\mathbf{C}^{n}\right)\right|_{S-C_{S}}$ which are orthogonal to the ideal generated by $\bar{\partial} \rho$ and $\phi$.

Let $Y$ be the vector field which is dual to $\phi$. This means that $Y\lrcorner \phi=1$ and $Y\lrcorner f=0$ for each $f \in \Lambda^{p, q}\left(\mathbf{C}^{n}\right)$ which is orthogonal to the ideal in $\Lambda^{*}\left(\mathbf{C}^{n}\right)$ generated by $\phi$. From the properties of contraction, we see that if $\left.f \in \Lambda^{p, q}\left(\mathbf{C}^{n}\right)\right|_{s-c_{s}}$ then $f \in \Lambda_{S}^{p, q}$ if and only if $\left.X\right\lrcorner\left. f\right|_{S}=0$ and $\left.Y\right\lrcorner\left. f\right|_{S}=0$.

Suppose $\left.f \in \Lambda_{M}^{p, q}\right|_{U \cap M}$; we define

$$
\left.f_{T_{s}}=Y\right\lrcorner\left.(\phi \wedge f) \in \Lambda_{M}^{p, q}\right|_{U \cap M}
$$

and

$$
\left.f_{N_{s}}=Y\right\lrcorner\left. f \in \Lambda_{M}^{p, q-1}\right|_{U \cap M} .
$$

Each section $\left.f \in \Lambda_{M}^{p, q}\right|_{U \cap M}$ has the orthogonal decomposition

$$
f=f_{T_{s}}+\phi \wedge f_{N_{s}}
$$

2.17. Proposition. Suppose $f \in \Lambda_{M}^{p, q}$; then $\left.f_{T_{S}}\right|_{s \cap U}=f_{t_{s}}$ is the complex tangential piece of $f$ along $S$ and $\left.f_{N_{S}}\right|_{S \cap U}$ defines an element of $\Lambda_{S}^{p, q-1}$.

Proof. It is easy to see that $\left.f_{T_{S}}\right|_{S \cap U} \in \Lambda_{S}^{p, q}$ because $\left.\left.X\right\lrcorner f_{T_{S}}=Y\right\lrcorner f_{T_{S}}=0$ on $S \cap U$. For example, we have

$$
\left.\left.\left.\left.\left.X\lrcorner f_{T_{s}}=-Y\right\lrcorner\{X\lrcorner(\phi \wedge f)\right\}=-Y\right\lrcorner\{(X\lrcorner \phi) \wedge f-\phi \wedge(X\lrcorner f\right)\right\} .
$$

Since $\left.\phi\right|_{M} \in \mathcal{E}_{M}^{0,1}$ and $f \in \Lambda_{M}^{p, q}$, we have $\left.X\right\lrcorner \phi=0$ and $\left.X\right\lrcorner f=0$. Therefore $\left.X\right\lrcorner f_{T_{s}}=$ 0 , as desired.

It is also clear that $\left.f_{T_{s}}\right|_{S \cap U}=\left.f\right|_{S \cap U}$ if and only if $\left.f\right|_{s \cap U} \in \Lambda_{S}^{p, q}$. This is because $\left.f_{T_{S}}=f-\phi \wedge(Y\lrcorner f\right)$ and $\left.Y\right\lrcorner\left. f\right|_{S \cap U}=0$ if and only if $f \in \Lambda_{S}^{p, q}$.

In a similar manner, one can show that $\left.f_{N_{s}}\right|_{s \cap U} \in \Lambda_{S}^{p, q-1}$ and $\left.f_{N_{S}}\right|_{s \cap U}=0$ if and only if $f \in \Lambda_{S}^{p, q}$. Q.E.D.

Terminology. We let $f_{n_{S}}=\left.f_{N_{S}}\right|_{S}$ and we call $f_{n_{S}}$ the complex normal piece of $f$ along $S$. Keep in mind that $f_{T_{S}}$ and $f_{N_{S}}$ are defined near $S-C_{S}$ but that $f_{t_{S}}$ and $f_{n_{S}}$ are defined only on $S-C_{S}$.

Restricting equation (2.15) to $S-C_{S}$ we obtain that each section $\left.f \in \Lambda_{M}^{p, q}\right|_{S-C_{S}}$ has an orthogonal decomposition:

$$
f=f_{t_{s}}+\phi \wedge f_{n_{s}}
$$

into its complex tangential and complex normal pieces along $S$.

2.19. Proposition. The following diagram commutes:

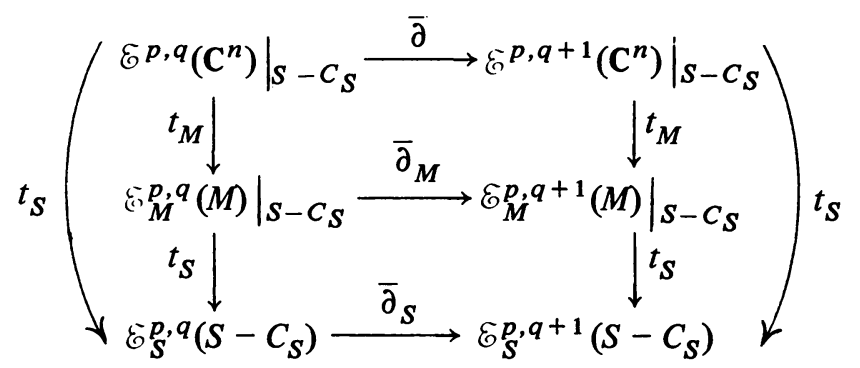


Proof. The only nontrivial part of the diagram is to show $\left(\bar{\partial}_{M} f\right)_{t_{s}}=\bar{\partial}_{S}\left(f_{t_{s}}\right)$ for $f \in \mathcal{E}_{M}^{p, q}$. If $\tilde{f}$ is any ambient extension of $f$, then $\bar{\partial}_{S}\left(f_{t_{s}}\right)=(\bar{\partial} \tilde{f})_{t_{s}}$. We note that if $\left.g \in \mathcal{E}^{*}\left(\mathbf{C}^{n}\right)\right|_{S-C_{s}}$, then $(g)_{t_{S}}=\left(g_{t_{M}}\right)_{t_{s}}$. Therefore, $\bar{\partial}_{S}\left(f_{t_{s}}\right)=\left((\bar{\partial} f)_{t_{M}}\right)_{t_{S}}=\left(\bar{\partial}_{M} f\right)_{t_{S}}$ as desired. Q.E.D.

2.2. Action of kernels. In this section, we briefly discuss kernels on an oriented smooth hypersurface $M$ in $\mathbf{C}^{\boldsymbol{n}}$. We follow the ideas put down in Harvey-Polking [6] and refer the reader to [6] for details not discussed here.

Suppose $U, V$ are open sets in $M$. We say that $E$ is a kernel on $U \times V$ if $E$ is a current on $U \times V$, i.e. $E(\zeta, z) \in \mathscr{D}_{M \times M}^{*}(U \times V)$. We view $E$ as representing an operator $E: \mathscr{D}_{M}^{*}(U) \rightarrow \mathscr{D}_{M}^{\prime *}(V)$ as follows: If $f \in \mathscr{D}_{M}^{*}(U)$ and $g \in \mathscr{D}_{M}^{*}(V)$, then

$$
(E(f), g)_{M}=(E, f \otimes g)_{M \times M}=\int_{z \in M} \int_{\zeta \in M} E(\zeta, z) \wedge f(\zeta) \wedge g(z) .
$$

Symbolically, we write $E(f)(z)=\left\{\int_{\zeta \in M} E(\zeta, z) \wedge f(\zeta)\right\}_{t_{M}}$ where we only integrate the piece of type $(n, n-1)$ in $\zeta$. This makes rigorous sense, for example, if $E(\zeta, z)$ has integrable coefficients on $M$ in $\zeta . E(\phi)(z)$ becomes a smooth form for $z \in V$ if $\{\operatorname{supp} \phi \times V\} \cap\{\operatorname{sing} \operatorname{supp} E\}=\varnothing$.

If $E \in \mathscr{D}_{M \times M}^{n, n-r}(U \times V)$, then it is not hard to see that $E: \mathscr{Q}_{M}^{p, q}(U) \rightarrow$ $\mathscr{Q}_{M}^{\prime p, q-r+1}(V)$.

We define the switching map, $s: M \times M \rightarrow M \times M$ by $s(\zeta, z)=(z, \zeta)$. The transpose of $E(\zeta, z)$, denoted by ${ }^{t} E(\zeta, z)$, is then defined by ${ }^{t} E=s^{*} E$. The transpose of $E$ is obtained by switching $\zeta$ with $z$ in the coefficients of $E$ and by switching $d \zeta$ and $d z$ and $d \bar{\zeta}$ with $d \bar{z}$. Up to a sign, ' $E$ is the current adjoint of $E$ on $M$. More precisely, suppose $E \in \mathscr{D}_{M \times M}^{n, n-r}(U \times V), f \in \mathscr{Q}_{M}^{p, q}(U)$, and $g \in$ $\mathscr{D}_{M}^{n-p, n-z+r}(V)$; then

$$
\left({ }^{t} E(f), g\right)_{M}=(-1)^{(p+q-1-r) r}(f, E(g))_{M} .
$$

For our purpose, the sign appearing here is rarely important.

2.20. Definition. Suppose $U, V$ are open sets in $M$ and suppose $E$ is a kernel on $U \times V$. We say $E$ is a regular kernel if $E(f)$ is smooth on $V$ whenever $f \in \mathscr{D}_{M}^{*}(U)$ and if $E$ represents a continuous operator $E: \mathscr{D}_{M}^{*}(U) \rightarrow \mathcal{E}_{M}^{*}(V)$. We say $E$ is biregular if both $E$ and ${ }^{t} E$ are regular kernels.

If $E$ is a biregular kernel on $M \times M$, then $E$ naturally extends to operate on currents on $M$. Suppose $S \subset M$ is an oriented hypersurface in $M$ and $E$ is a biregular kernel on $M \times M$. If $f \in \mathscr{D}_{S}^{*}\left(S-C_{S}\right)$, then $E\left([S]^{0,1} \wedge f\right)$ is defined as a current on $M$. Formally, we write

$$
E\left([S]^{0,1} \wedge f\right)(z)=\left\{\int_{\zeta \in S} E(\zeta, z) \wedge f(\zeta)\right\}_{t_{M}}
$$

where we only integrate the piece of type $(n, n-2)$ in $\zeta$. Regardless of whether or not $E$ is a biregular kernel, (2.21) makes sense and defines a smooth form for $z \in U$, provided that $\{S \times U\} \cap\{\operatorname{sing} \operatorname{supp} E\}=\varnothing$.

It is clear from the above conventions that if

$$
E \in \mathscr{D}_{M \times M}^{n, n-r} \text { and } f \in \mathscr{Q}_{S}^{p, q}\left(S-C_{S}\right) \text {, }
$$


then $E\left([S]^{0,1} \wedge f\right) \in \mathscr{D}_{M}^{p, q-r+2}$. In particular, if $r=2$, then the operation of sending $f$ to $E\left([S]^{0,1} \wedge f\right)$ preserves the bidegree of $f$.

2.3. Formal identities. In this section, we describe the kernels we shall use and establish some formal identities that relate these kernels to the $\bar{\partial}_{M}$ operator. We closely follow the development given in [6].

Let $M$ be a hypersurface in $C^{n}$ and let $V$ be an open set in $M \times M$ with coordinates $(\zeta, z)$. Suppose $u^{j}(\zeta, z)=\left(u_{1}^{j}(\zeta, z), \ldots, u_{n}^{j}(\zeta, z)\right)$ is a smooth mapping $u^{j}: V \rightarrow \mathbf{C}^{n}$ for $1 \leqslant j \leqslant N$. We adopt the following notation from [6]:

$$
\begin{gathered}
u^{j}(\zeta, z) \cdot(\zeta-z)=\sum_{k=1}^{n} u_{k}^{j}(\zeta, z)\left(\zeta_{k}-z_{k}\right), \\
u^{j}(\zeta, z) \cdot d(\zeta-z)=\sum_{k=1}^{n} u_{k}^{j}(\zeta, z) d\left(\zeta_{k}-z_{k}\right), \\
\left(\bar{\partial}_{M} u^{j}\right)(\zeta, z) \cdot d(\zeta-z)=\sum_{k=1}^{n}\left(\bar{\partial}_{M} u_{k}^{j}\right)(\zeta, z) \wedge d\left(\zeta_{k}-z_{k}\right),
\end{gathered}
$$

where $\bar{\partial}_{M}$ is taken in both variables, $\zeta$ and $z$. We define the following one forms on $V$ :

$$
\omega_{j}=\omega_{j}\left(u^{j}\right)=\frac{u^{j}(\zeta, z) \cdot d(\zeta-z)}{u^{j}(\zeta, z) \cdot(\zeta-z)}, \quad 1 \leqslant j \leqslant N .
$$

Each $\omega_{j}$ is smooth on $V-A_{j}$ where $A_{j}=\left\{(\zeta, z) \in V ; u^{j}(\zeta, z) \cdot(\zeta-z)=0\right\}$. It is clear that $\omega_{j}$ is homogeneous of degree zero; that is if $h_{j}: V \rightarrow \mathbf{C}$ is a smooth function then

$$
\omega_{j}\left(h_{j} u^{j}\right)=\omega_{j}\left(u^{j}\right) \text { for each } j .
$$

2.23. Proposition. For each integer $k \geqslant 1$ we have

$$
\omega_{j}\left(u^{j}\right) \wedge\left[\bar{\partial}_{M} \omega_{j}\left(u^{j}\right)\right]^{k}=\left[\frac{u^{j} \cdot d(\zeta-z)}{u^{j} \cdot(\zeta-z)}\right] \wedge\left[\frac{\bar{\partial}_{M} u^{j} \cdot d(\zeta-z)}{u^{j} \cdot(\zeta-z)}\right]^{k} .
$$

Proof. The proof is clear after noting that

$$
\bar{\partial}_{M} \omega_{j}\left(u^{j}\right)=\left[\frac{\bar{\partial}_{M} u^{j} \cdot d(\zeta-z)}{u^{j} \cdot(\zeta-z)}\right]-\frac{\left(\bar{\partial}_{M} u^{j} \cdot(\zeta-z)\right) \wedge\left(u^{j} \cdot d(\zeta-z)\right)}{\left(u^{j} \cdot(\zeta-z)\right)^{2}} \text { Q.E.D. }
$$

To state our formal identities, we shall use the following multi-index notation adopted from [6].

If $\alpha=\left(\alpha_{1}, \ldots, \alpha_{N}\right)$ is an $N$-tuple of nonnegative integers, then we let $|\alpha|=\alpha_{1}$ $+\cdots+\alpha_{N}$ and $\left(\bar{\partial}_{M} \omega\right)^{\alpha}=\left(\bar{\partial}_{M} \omega_{1}\right)^{\alpha_{1}} \wedge \cdots \wedge\left(\bar{\partial}_{M} \omega_{N}\right)^{\alpha_{N}}$. If $I=\left\{i_{1}, \ldots, i_{p}\right\}$ is a $p$-tuple of positive integers then we let $\omega_{I}=\omega_{i_{1}} \wedge \cdots \wedge \omega_{i_{p}}$ and $E_{I}\left(u^{i_{1}}, \ldots, u^{i_{j}}\right)=$ $(2 \pi i)^{-n} \omega_{I} \wedge \Sigma_{|\alpha|=n-p}\left(\bar{\partial}_{M} \omega\right)^{\alpha}$. If the $u^{i}$ are understood then we just write $E_{I}$ for $E_{I}\left(u^{i_{1}}, \ldots, u^{i_{p}}\right)$. The form $E_{I}$ is a smooth form on $V-\left\{\cup_{j=1}^{N} A_{j}\right\}$ and it is clear from (2.22) that $E_{I}$ is homogeneous of degree zero, i.e. we have the following proposition: 
2.24. Proposition. Suppose $h_{j}: V \rightarrow \mathrm{C}, 1 \leqslant j \leqslant N$, are smooth functions. Then

$$
E_{I}\left(h_{i_{1}} u^{i_{1}}, \ldots, h_{i_{p}} u_{p}^{i_{p}}\right)=E_{I}\left(u^{i_{1}}, \ldots, u^{i_{p}}\right) .
$$

For $1 \leqslant j \leqslant p$, we set $I_{j}=\left\{i_{1}, \ldots, \hat{i_{j}}, \ldots, i_{p}\right\}$ where we mean that the element $i_{j}$ is omitted.

The identity we need is the following:

2.25. TheOREM. On the set $V-\left\{\cup_{j=1}^{N} A_{j}\right\}$, we have

$$
\bar{\partial}_{M} E_{I}=\left\{\sum_{j=1}^{p}(-1)^{j} E_{i j}\right\}_{t_{M \times M}}
$$

where, as before, $\bar{\partial}_{M}$ is taken in both $\zeta$ and $z$.

Proof. In Harvey-Polking [6] the following formula is proved:

$$
\bar{\partial} E_{I}=\sum_{j=1}^{p}(-1)^{j} E_{i_{j}} .
$$

Therefore, Theorem 2.25 follows after applying the map $t_{M \times M}$ Q.E.D.

In the applications that follow, we will let $p=N=3, I=(1,2,3), u^{1}(\zeta, z)=$ $u(\zeta, z), u^{2}(\zeta, z)={ }^{t} u(\zeta, z)=u(z, \zeta)$, and $u^{3}(\zeta, z)=v(\zeta, z)$ where $u$ and $v$ are smooth functions to be constructed later. We get the corresponding kernels $E_{123}, E_{12}, E_{13}, E_{23}, E_{1}, E_{2}$, and $E_{3}$. We shall let $R=E_{12}$ and $L=E_{1}$. Since $u^{2}(\zeta, z)$ $={ }^{t} u^{1}(\zeta, z)$, it is easy to see that $E_{2}={ }^{t} E_{1}={ }^{t} L$. Using Proposition 2.23 , we can write out these kernels explicitly. For example, we have

$$
\begin{aligned}
R(\zeta, z)=(2 \pi i)^{-n}\{ & \left(\frac{u \cdot d(\zeta-z)}{u \cdot(\zeta-z)}\right) \wedge\left(\frac{{ }^{t} u \cdot d(\zeta-z)}{{ }^{t} u \cdot(\zeta-z)}\right) \\
& \left.\wedge \sum_{j+k=n-2}\left(\frac{\bar{\partial} u \cdot d(\zeta-z)}{u \cdot(\zeta-z)}\right)^{j}\left(\frac{\bar{\partial}^{t} u \cdot d(\zeta-z)}{{ }^{t} u \cdot(\zeta-z)}\right)^{k}\right\}_{t_{M \times M}} .
\end{aligned}
$$

Theorem 2.25 implies the following identities

$$
\begin{aligned}
\bar{\partial}_{M} E_{123} & =E_{13}-E_{23}+R, \\
\bar{\partial}_{M} R & =L-{ }^{t} L, \\
\bar{\partial}_{M} E_{13} & =E_{3}-L, \\
\bar{\partial}_{M} E_{23} & =E_{3}-{ }^{t} L .
\end{aligned}
$$

These identities hold off the singular set $A=A_{1} \cup A_{2} \cup A_{3}$. In our applications we will take $v(\zeta, z)$ to be holomorphic in $\zeta$ and $z$. Hence, $\bar{\partial} v=0$ and so $E_{3}$ will vanish.

In Chapter 4, we will take principal value limits of these kernels across the singular set $A$ and we will be particularly interested in what happens to equations (2.27) and (2.28), after we take principal value limits. 
Chapter 3. The global SOlution to $\bar{\partial}_{M}$ ON A STRICTLY PSEUDOCONVEX HYPERSURFACE

On a strictly convex, or more generally a strictly pseudoconvex hypersurface $M$, there exists a support function $u(\zeta, z)$ such that the $R$ kernel, defined in (2.26), is a fundamental solution to $\bar{\partial}_{M}$. This kernel was discovered by Romanov and, independently, Skoda. In this chapter we outline the construction of the support function $u(\zeta, z)$ and review the facts we need about the $R$ kernel.

For simplicity in stating estimates, we shall adopt the following notation:

Suppose $\mathbf{C}^{n} \times \mathbf{C}^{n}$ is given coordinates $(w, z)$ and $f: \mathbf{C}^{n} \times \mathbf{C}^{n} \rightarrow \mathbf{C}$ is a smooth function. We say that:

$$
f(w, z)=\mathcal{O}\left(|w|^{k}\right)
$$

if there exist smooth functions $a_{\alpha \beta}(w, z)$ such that

$$
f(w, z)=\sum_{|\alpha|+|\beta|=k} a_{\alpha \beta}(w, z) w^{\alpha} \bar{w}^{\beta} .
$$

Note that if $X_{z}$ is a vector field in $z$, then

$$
X_{z} f(w, z)=\sum_{|\alpha|+|\beta|=k} X_{z} a_{\alpha}(w, z) w^{\alpha} \bar{w}^{\beta}=\mathcal{O}\left(|w|^{k}\right) .
$$

We say that

$$
|f(w, z)| \gtrsim|w|^{k} \quad \text { for } z \text { in a set } K
$$

if there exists a constant $C$ independent of $z \in K$ such that $|f(w, z)|>C|w|^{k}$ for $|w|$ suitably small.

We say that

$$
f(w, z) \approx|w|^{k} \quad \text { for } z \in K
$$

if both $f(w, z)=\theta\left(|w|^{k}\right)$ and $|f(w, z)| \gtrsim|w|^{k}$ for $z \in K$ are satisfied.

Now suppose $D \subset \mathbf{C}^{n}$ is an open set. We denote the complement of $D$ by $D^{c}$.

3.4. Defintrion. Suppose $u(\zeta, z): N \rightarrow \mathrm{C}^{n}$ is a smooth function, where $N$ is some neighborhood of $\partial D \times \partial D$ in $\mathbf{C}^{n} \times \mathbf{C}^{n}$. We say that $u$ is a strong support function for $\partial D$ if

(a) $u(\zeta, z) \cdot(\zeta-z) \neq 0$ for $(\zeta, z) \in N \cap\left\{\overline{D^{c}} \times \bar{D}\right\}-\Delta$.

(b) $u(\zeta, z)$ is holomorphic in $z \in D$ for each fixed $\zeta$.

(c) Given any compact set $K \subset \partial D \times \partial D$, then $\operatorname{Re}\{u(\zeta, z) \cdot(\zeta-z)\} \approx|\zeta-z|^{2}$ for $(\zeta, z) \in K$ and $|\zeta-z|$ suitably small.

Note that if $u$ is a strong support function for $\partial D$, then ${ }^{t} u(\zeta, z)=u(z, \zeta)$ satisfies

(3.5) (a) ${ }^{t} u(\zeta, z) \cdot(\zeta-z) \neq 0$ for $(\zeta, z) \in N \cap\left\{\bar{D} \times \overline{D^{c}}\right\}-\Delta$.

(b) ' $u(\zeta, z) \cdot(\zeta-z)$ is holomorphic in $\zeta \in D$ for each fixed $z$.

(c) Given any compact set $K \subset \partial D \times \partial D$, then $\operatorname{Re}\left\{{ }^{t} u(\zeta, z) \cdot(\zeta-z)\right\} \approx|\zeta-z|^{2}$ for $(\zeta, z) \in K$ with $|\zeta-z|$ suitably small.

We now want to show that if $D$ is strictly convex or strictly pseudoconvex, then there exists a support function for $M=\partial D$. 
3.6. Definition. (a) $M=\partial D$ is strictly convex if the defining function for $M$, $\rho$, is a strictly convex function, i.e. the real hessian of $\rho$ is positive definite on the tangent space of $M$.

(b) $M=\partial D$ is strictly pseudoconvex if the complex hessian $\partial^{2} \rho(\zeta) / \partial \zeta_{j} \partial \bar{\zeta}_{k}$ of the defining function is positive definite on the holomorphic tangent space of $M$ at $\zeta$.

3.7. LEMMA. (a) Suppose $M=\partial D$ is strictly pseudoconvex; then $u(\zeta, z)=\partial \rho(\zeta) / \partial \zeta$ $=\left(\partial \rho(\zeta) / \partial \zeta_{1}, \ldots, \partial \rho(\zeta) / \partial \zeta_{n}\right)$ is a strong support function for $\boldsymbol{M}$.

(b) Suppose $M=\partial D$ is strictly pseudoconvex; then there is a strong support function $u(\zeta, z)$ for $M$ defined in a neighborhood of $\partial D \times \partial D$. In addition

$$
u(\zeta, z) \cdot(\zeta-z)=h(\zeta, z) \hat{u}(\zeta, z) \cdot(\zeta-z)
$$

for $(\zeta, z)$ near $\Delta \cap\{\partial D \times \partial D\}$ where $h(\zeta, z)$ is a smooth nonvanishing function which is holomorphic in $z$ for each fixed $\zeta$ and

$$
\hat{u}_{j}(\zeta, z)=\frac{\partial \rho}{\partial \zeta_{j}}(\zeta)-\frac{1}{2} \sum_{k=1}^{n} \frac{\partial^{2} \rho}{\partial \zeta_{j} \partial \zeta_{k}}(\zeta)\left(\zeta_{k}-z_{k}\right), \quad 1<j<n .
$$

Proof. (a) Since $u(\zeta, z)$ is independent of $z$, it is clear that part (b) of Definition 3.4 holds for $u(\zeta, z)$. Part (a) of Definition 3.4 follows from the convexity of $M$, but we do not need the full strength of strict convexity (see Harvey-Polking [6, Proposition 6.2] for more details).

To prove that $u(\zeta, z)$ satisfies part (c) of 3.4, we look at a Taylor series expansion of $\rho$ about the point $\zeta$.

$$
\rho(z)=\rho(\zeta)-2 \operatorname{Re}\{u(\zeta, z) \cdot(\zeta-z)\}+P(\zeta, z)
$$

where $P(\zeta, z)$ is the Taylor remainder of second order. Since $\rho$ is strictly convex, the remainder satisfies $P(\zeta, z) \approx|\zeta-z|^{2}$ for $(\zeta, z) \in K$ and $|\zeta-z|$ suitably small. Therefore, if $(\zeta, z) \in M \times M$, then:

$$
2 \operatorname{Re}\{u(\zeta, z) \cdot(\zeta-z)\}=P(\zeta, z) \approx|\zeta-z|^{2}
$$

and property (c) is satisfied.

(b) Part (b) is due to Henkin [7]; see also Ramirez de Arellano [15] and Overlid [14]. Q.E.D.

Suppose $M=\partial D$ is strictly pseudoconvex and $u$ is a strong support function for $M$. For fixed $z \in \mathbf{C}^{n}$, let:

$$
\begin{aligned}
& A_{z}=\left\{\zeta \in \mathbf{C}^{n} ; u(\zeta, z) \cdot(\zeta-z)=0\right\}, \\
& { }^{t} A_{z}=\left\{\zeta \in \mathbf{C}^{n} ;{ }^{t} u(\zeta, z) \cdot(\zeta-z)=0\right\} .
\end{aligned}
$$

Part (a) of 3.4 and (3.5) imply that if $z \in M$, then $A_{z} \cap M={ }^{t} A_{z} \cap M=\{z\}$. Therefore, the singular sets in $M \times M$ of $R(\zeta, z), L(\zeta, z),{ }^{t} L(\zeta, z)$ are just the diagonal of $M \times M$, cf. formula (2.26), etc.

Since $\operatorname{Re} u(\zeta, z) \cdot(\zeta-z) \approx|\zeta-z|^{2}$ for $(\zeta, z) \in M \times M$ and $|\zeta-z|$ suitably small, it can be shown that the $R(\zeta, z)$ kernel is integrable on $M$ in each variable separately (see $\S 4$, also [6, Theorem 9.13]). Therefore, if $f \in \mathcal{E}_{M}^{\prime p, q}$ is a current on $M$ with $L^{\infty}$ coefficients, then $R(f)(z)=\int_{\zeta \in M} R(\zeta, z) \wedge f(\zeta)$ is a form on $M$ with continuous coefficients. In addition we have 
3.8. TheOREM. If $f \in \mathscr{Q}_{M}^{p, q}$ is a smooth form on $M$, then $R(f) \in \mathcal{E}_{M}^{p, q-1}$ is smooth. Furthermore $R$ represents a continuous operator $R: \mathscr{Q}_{M}^{p, q} \rightarrow \mathcal{E}_{M}^{p, q-1}$.

We defer the proof until \$4.3. Since ${ }^{t} R=R$, the $R$ kernel is selfadjoint up to sign and hence the $R$ kernel is a biregular kernel on $M \times M$.

Unfortunately, the kernels $L$ and ${ }^{t} L$, given in Chapter 2, are not integrable on $M$. However, in view of 3.4(a), it is clear that if $f \in \mathscr{Q}_{M}^{p, q}$, then $L(f)(z)=$ $\int_{\zeta \in M} L(\zeta, z) \wedge f(\zeta)$ defines a smooth form for $z \in D$. From (3.5)(b), it is also clear that if $f \in \mathscr{O}_{M}^{p, q}$ then ${ }^{t} L(f)(z)$ defines a smooth form for $z \in D^{c}$. Harvey and Polking have shown (see [6, Theorem 8.1]) that the smooth boundary values of $L(f)$ (resp. ${ }^{t} L(f)$ ) from $D$ (resp. $D^{c}$ ) exist on $M$ and define a smooth form on $M$ which we denote by $L_{M}(f)$ (resp. $\left.{ }^{t} L_{M}(f)\right)$. In view of type considerations, it is clear that $L_{M}(f)=0$ unless $f \in \mathcal{E}_{M}^{p, 0}$ and ${ }^{t} L_{M}(f)=0$ unless $f \in \mathcal{E}_{M}^{p, n-1}$. Since $u(\zeta, z)$ is holomorphic in $z$, it is also clear that $\bar{\partial}_{M}\left\{L_{M}(f)\right\}=0$ if $f \in \mathcal{E}_{M}^{p, 0}$.

3.9. TheOREM (Henkin). Suppose $g \in \mathscr{P}_{M}^{p, q}, 0 \leqslant q \leqslant n-1$; then

$$
\bar{\partial}_{M}\{R(g)\}+R\left(\bar{\partial}_{M} g\right)=g+\left(L_{M}-{ }^{t} L_{M}\right)(g) .
$$

Proof. See Henkin [10], also [6].

Formula (3.10) breaks down into two cases of interest to us:

(3.11) If $g \in \mathscr{Q}_{M}^{p, q}, 1 \leqslant q \leqslant n-2$, then $\bar{\partial}_{M}\{R(g)\}+R\left(\bar{\partial}_{M} g\right)=g$.

(3.12) If $g \in \mathscr{Q}_{M}^{p, n-1}$ then $\bar{\partial}_{M}\{R(g)\}=g-{ }^{t} L_{M}(g)$.

Since the $R$ kernel is biregular on $M$, it is clear that (3.11) holds if $g$ is a current on $M$.

Now, suppose $S$ is an oriented smooth hypersurface in $M$.

3.13. Definition. Suppose $f \in \mathscr{Q}_{S}^{p, n-2}\left(S-C_{S}\right)$. We say $f$ satisfies condition $\theta$ for $S$ if $\int_{\zeta \in M} f(\zeta) \wedge g(\zeta)=0$ for all $g \in \mathcal{E}_{S}^{n-p, 0}$ with $\bar{\partial}_{S} g=0$ near $\{\operatorname{supp} f\}$.

3.14. TheOREM. (a) Suppose $\left.f \in \mathscr{D}_{M}^{p, q}\right|_{S}, 0 \leqslant q \leqslant n-2$; then $R\left([S]^{0,1} \wedge f\right)(z)=$ $\left\{\int_{\zeta \in S} R(\zeta, z) \wedge f(\zeta)\right\}_{t_{M}}$ defines a current on $M$ with smooth coefficients on $M-S$.

(b) Suppose $f \in \mathscr{D}_{S}^{p, q}\left(S-C_{S}\right), 0 \leqslant q \leqslant n-3$; then

$$
\bar{\partial}_{M}\left\{R\left([S]^{0,1} \wedge f\right)\right\}-R\left([S]^{0,1} \wedge \bar{\partial}_{S} f\right)=[S]^{0,1} \wedge f
$$

(c) Suppose $f \in \mathscr{D}_{S}^{p, n-2}\left(S-C_{S}\right)$ and satisfies condition $\theta$ for $S$; then

$$
\bar{\partial}_{M}\left\{R\left([S]^{0,1} \wedge f\right)\right\}=[S]^{0,1} \wedge f
$$

Proof. (a) Since the singular set in $M \times M$ of the $R$ kernel is the diagonal, it is clear that $R\left([S]^{0,1} \wedge f\right)(z)$ is smooth for $z \in M-S$.

(b) To prove part (b), we apply (3.11) with $g=[S]^{0,1} \wedge f$ and note that $\bar{\partial}_{M} g=-[S]^{0,1} \wedge \bar{\partial}_{S} f$.

(c) Let $\eta_{t}$ be a sequence of smooth functions such that $\bar{\partial}_{M} \eta_{t} \rightarrow[S]^{0,1}$ as $t \rightarrow 0$, in the sense of currents. We apply (3.12) to $g=\bar{\partial}_{M} \eta_{t} \wedge f$ and obtain

$$
\bar{\partial}_{M}\left\{R\left(\bar{\partial}_{M} \eta_{t} \wedge f\right)\right\}=\bar{\partial}_{M} \eta_{t} \wedge f-{ }^{t} L_{M}\left(\bar{\partial}_{M} \eta_{t} \wedge f\right) .
$$


We claim that ${ }^{t} L_{M}\left(\bar{\partial}_{M} \eta_{t} \wedge f\right) \rightarrow 0$ as $t \rightarrow 0$ in the sense of currents. To see this, let $h \in \mathcal{E}_{M}^{n-p, 0}$. Then

$$
\left({ }^{t} L_{M}\left(\bar{\partial}_{M} \eta_{t} \wedge f\right), h\right)_{M}=(-1)^{n-p+1}\left(\bar{\partial}_{M} \eta_{t} \wedge f, L_{M}(h)\right)_{M}
$$

which converges, as $t \rightarrow 0$, to

$$
(-1)^{n-p+1}\left([S]^{0,1} \wedge f, L_{M}(h)\right)_{M}=(-1)^{n-p+1} \int_{\zeta \in S} f(\zeta) \wedge L_{M}(h)(\zeta) .
$$

Since $\bar{\partial}_{M}\left\{L_{M}(h)\right\}=0,(3.18)$ vanishes because $f$ satisfies condition $\theta$ for $S$.

Now we may let $t \rightarrow 0$ in equation (3.17) to establish part (c). Q.E.D.

\section{ChaPTER 4. THE LOCAL SOlution to $\bar{\partial}_{M}$}

In Chapter 3, we stated that the $R$-kernel globally solves $\bar{\partial}_{M}$, where $M=\partial D$ is the boundary of a strictly pseudoconvex domain, $D \subset C^{n}$. In this chapter, we solve three theorems concerning the local solution to $\bar{\partial}_{M}$. These theorems will be proven after taking principal value limits across the singular sets of the kernels defined at the end of Chapter 2.

4.1. Regular generating functions and principal value limits. Let $M=\partial D$ where $D$ is a strictly pseudoconvex domain in $\mathbf{C}^{n}$. Suppose $V$ is an open subset of $M \times M$ with coordinates $(\zeta, z)$ and let $v: V \rightarrow \mathbf{C}^{n}$ be a smooth mapping.

4.1. Definition. The function $v$ is said to be a regular generating function for $V$ if:

(a) For each $\left(z_{0}, z_{0}\right) \in V$, the matrix

$$
\left(\begin{array}{l}
(\nabla \rho)\left(z_{0}\right) \\
J\left\{\nabla \rho\left(z_{0}\right)\right\} \\
\left.\nabla_{\zeta}\left\{\operatorname{Re} v\left(\zeta, z_{0}\right) \cdot\left(\zeta-z_{0}\right)\right\}\right|_{\zeta=z_{0}} \\
\left.\nabla_{\zeta}\left\{\operatorname{Im} v\left(\zeta, z_{0}\right) \cdot\left(\zeta-z_{0}\right)\right\}\right|_{\zeta=z_{0}}
\end{array}\right)
$$

has maximal rank, where $J: \mathbf{R}^{2 n} \rightarrow \mathbf{R}^{2 n}$ is the linear map induced by multiplication by $i$.

(b) For each $\left(\zeta_{0}, z_{0}\right) \in V$ with $v\left(\zeta_{0}, z_{0}\right) \cdot\left(\zeta_{0}-z_{0}\right)=0$, the matrix

$$
\left[\begin{array}{l}
\left(\nabla_{\rho}\right)\left(\zeta_{0}\right) \\
\left.\nabla_{\zeta}\left\{\operatorname{Re} v\left(\zeta, z_{0}\right) \cdot\left(\zeta-z_{0}\right)\right\}\right|_{\zeta=z_{0}} \\
\left.\nabla_{\zeta}\left\{\operatorname{Im} v\left(\zeta, z_{0}\right) \cdot\left(\zeta-z_{0}\right)\right\}\right|_{\zeta=z_{0}}
\end{array}\right]
$$

has maximal rank.

Note that the conditions in Definition 4.1 are open conditions, i.e. if $v$ satisfies these conditions on a set $S \subset M \times M$, then they will be satisfied near $S$ in $M \times M$.

Let $u$ be a strong support function for $M$, and let $v$ be a regular generating function for $V \subset M \times M$. We define the following one forms

$$
\omega_{1}=\frac{u(\zeta, z) \cdot d(\zeta-z)}{u(\zeta, z) \cdot(\zeta-z)}, \quad \omega_{2}=\frac{{ }^{t} u(\zeta, z) \cdot d(\zeta-z)}{{ }^{t} u(\zeta, z) \cdot(\zeta-z)}, \quad \omega_{3}=\frac{v(\zeta, z) \cdot d(\zeta-z)}{v(\zeta, z) \cdot(\zeta-z)},
$$


and we get the kernels $E_{123}, E_{13}, E_{23}, R, L$ and ${ }^{t} L$ as defined in Chapter 2. These kernels are smooth on $V-B$, where $B=\{(\zeta, z) \in V ; v(\zeta, z) \cdot(\zeta-z)=0\}$. Theorem 2.25 yields

$$
\bar{\partial}_{M} E_{123}=-E_{23}+E_{13}-R \text { on } V-B,
$$

where on the left, $\bar{\partial}_{M}$ is taken in both variables.

For $\varepsilon>0$, we define

$$
\chi_{\varepsilon}= \begin{cases}1 & \text { if }|v(\zeta, z) \cdot(\zeta-z)| \geqslant \varepsilon \\ 0 & \text { otherwise. }\end{cases}
$$

For each $\varepsilon>0$, the currents $\chi_{e} E_{123}, \chi_{e} E_{13}$, and $\chi_{e} E_{23}$ define kernels on $V$. The following theorem is our main result concerning the principal value limits of our kernels.

4.3. THEOREM. Suppose $v$ is a regular generating function for $V \subset M \times M$.

(a) The currents $E_{123}, E_{13}$, and $E_{23}$ define principal value currents on $V$ by the formulas

$$
\begin{aligned}
& \text { P.V. } E_{123}=\lim _{\varepsilon \rightarrow 0} \chi_{\varepsilon} E_{123}, \\
& \text { P.V. } E_{13}=\lim _{\varepsilon \rightarrow 0} \chi_{\varepsilon} E_{13}, \\
& \text { P.V. } E_{23}=\lim _{\varepsilon \rightarrow 0} \chi_{\varepsilon} E_{23} .
\end{aligned}
$$

Furthermore, the limit $S=\lim _{\varepsilon \rightarrow 0}\left(\bar{\partial}_{M} \chi_{e}\right) \wedge E_{123}$ exists and defines a current $S \in$ $\mathscr{D}_{M \times M}^{n, n-2}$ with supp $S \subset B$.

(b) Suppose $U_{1}, U_{2}$ are open sets in $M$ with $U_{1} \times U_{2} \subset V$. Suppose $g \in \mathscr{D}_{M}^{*}\left(U_{1}\right)$; then for each $\varepsilon>0$, the forms $\left(\chi_{e} E_{123}\right)(g),\left(\chi_{e} E_{13}\right)(g),\left(\chi_{e} E_{23}\right)(g)$ and $\left(\bar{\partial}_{M} \chi_{\varepsilon} \wedge E_{123}\right)(g)$ are smooth on $U_{2}$ and they converge (in the topology of $\mathcal{E}\left(U_{2}\right)$ ) as $\varepsilon \rightarrow 0^{+}$to (P.V. $\left.E_{123}\right)(g)$, (P.V. $\left.E_{13}\right)(g)$, (P.V. $\left.E_{23}\right)(g)$, and $S(g)$, respectively. In particular, the kernels P.V. $E_{123}$, P.V. $E_{13}$, P.V. $E_{23}$, and $S$ are regular kernels on $U_{1} \times U_{2}$.

(c) The following current equations hold on $V$ :

$$
\begin{gathered}
\bar{\partial}_{M}\left\{\text { P.V. } E_{123}\right\}=\text { P.V. } E_{13}-\text { P.V. } E_{23}-R+S, \\
\bar{\partial}_{M}\left\{\text { P.V. } E_{13}-\text { P.V. } E_{23}+S\right\}=-[\Delta]+L_{M}-{ }^{t} L_{M},
\end{gathered}
$$

where $[\Delta]$ is the current 'integration over the diagonal of $V$ '.

Remark. Equation (4.5) implies that the current P.V. $E_{13}-$ P.V. $E_{23}+S$ is a fundamental solution for $\bar{\partial}_{M}$ on $V$ in the following sense. Suppose $U \times U \subset V$ and $g \in \mathscr{Q}_{M}^{p, q}(U), 1 \leqslant q \leqslant n-2$, with $\bar{\partial}_{M} g \equiv 0$. Then

$$
\bar{\partial}_{M}\left\{\left(\text { P.V. } E_{13}-\text { P.V. } E_{23}+S\right)(g)\right\}=g \text { on } U .
$$

Proof. We shall prove the easy parts here and prove the more technical parts in §4.3. Suppose we knew the principal value limits of $E_{123}, E_{13}$, and $E_{23}$ exist and define regular kernels on $U_{1} \times U_{2}$. Then, the rest of the theorem follows easily. For example, by (4.2), we have

$$
\bar{\partial}_{M}\left\{\chi_{e} E_{123}\right\}=\chi_{e}\left(E_{13}-E_{23}-R\right)+\bar{\partial}_{M} \chi_{e} \wedge E_{123}
$$


which holds on $V$. Since $R(\zeta, z)$ is integrable on $M \times M$ (Theorem 3.8), we have $\lim _{\varepsilon \rightarrow 0} \chi_{\varepsilon} R=R$. Therefore, we may let $\varepsilon \rightarrow 0$ in the above equation to obtain

$$
\bar{\partial}_{M}\left\{\text { P.V. } E_{123}\right\}=\text { P.V. } E_{13}-\text { P.V. } E_{23}-R+\lim _{\varepsilon \rightarrow 0}\left\{\bar{\partial}_{M} \chi_{\ell} \wedge E_{123}\right\} .
$$

Since we are assuming that P.V. $E_{123}$, P.V. $E_{13}$ and P.V. $E_{23}$ are well-defined currents, we conclude that $S=\lim _{\varepsilon \rightarrow 0}\left(\bar{\partial}_{M} \chi_{e}\right) \wedge E_{123}$ defines a current on $V$ and that (4.4) is valid on $V$. Equation (4.5) now follows by taking $\bar{\partial}_{M}$ of both sides of equation (4.4) and by using Theorem 3.9 for the $R$ kernel.

Since the $R$ kernel is regular on $M \times M$ and since we are assuming the kernels P.V. $E_{123}$, P.V. $E_{13}$ and P.V. $E_{23}$ are regular kernels on $U_{1} \times U_{2}$, equation (4.4) implies the kernel $S$ is also regular on $U_{1} \times U_{2}$.

It is clear that supp $S \subset B$ because for each $\varepsilon>0$,

$$
\operatorname{supp} \bar{\partial}_{M} \chi_{\varepsilon}=\{(\zeta, z) \in V ;|v(\zeta, z) \cdot(\zeta-z)|=\varepsilon\}
$$

So, it suffices to prove that P.V. $E_{123}$, P.V. $E_{13}$ and P.V. $E_{23}$ define regular kernels on $U_{1} \times U_{2}$. This is done in $\S 4.3$. Q.E.D.

REMARK. It is clear that on $V-B$ (i.e. away from the singular sets of these kernels) we have: P.V. $E_{123}=E_{123}$, P.V. $E_{13}=E_{13}$, and P.V. $E_{23}=E_{23}$. So for simplicity, we shall denote the kernels P.V. $E_{123}$, P.V. $E_{13}$, and P.V. $E_{23}$ by $E_{123}, E_{13}$, and $E_{23}$, respectively.

4.2. Three theorems on the local solution to $\bar{\partial}_{M}$. In this section we use Theorem 4.3 to solve three theorems concerning the local solution to $\bar{\partial}_{M}$. In this section, $M=\partial D$ is the boundary of a strictly pseudoconvex domain $D \subset \mathbf{C}^{n}$ with defining function $\rho$. We let $\omega \subset \subset M$ be an open subset of $M$ defined by $\omega=\{z \in M$; $r(z)=\operatorname{Re} h(z)<0\}$ where $h$ is a holomorphic function on a neighborhood of $\bar{D}$. We also assume that $\omega_{t}=\{z \in M ; r(z)<t\}$ has compact closure for each $t$ near zero.

We let $C=C_{\partial \omega}$ be the set of characteristic points on $\partial \omega$.

4.6. Definition. $\partial \omega$ is said to be an admissible boundary if whenever $z \in \partial \omega-C$, then $B_{z} \cap C=\varnothing$ where $B_{z}=\{\zeta \in M ; h(\zeta)=h(z)\}$.

ExAMPLes. Let $M$ be the unit sphere in $C^{n}$ and let $\omega=\left\{z \in M ; \operatorname{Re} z_{1}>0\right\}$ be the upper hemisphere. Then $\partial \omega=\left\{z \in M ; \operatorname{Re} z_{1}=0\right\}$ is the equator and $C=C_{\partial \omega}$ $=\{( \pm i, 0, \ldots, 0)\}$. We see that $h(z)=-z_{1}$ and so $B_{z}=\left\{\zeta \in M ; \zeta_{1}=z_{1}\right\}$. In fact, for $z \in \partial \omega, B_{z}$ is a sphere of real dimension $2 n-3$ with radius $\sqrt{1-\left(\operatorname{Im} z_{1}\right)^{2}}$ and with center at $\left(i \operatorname{Im} z_{1}, 0, \ldots, 0\right)$. Therefore, it is clear that $\partial \omega$ is admissible.

The previous example can be generalized to any strictly convex boundary $M$ oriented so that the plane $\left\{z ; \operatorname{Re} z_{1}=0\right\}$ intersects $M$ transversally. Let $\omega=\{z \in$ $\left.M ; \operatorname{Re} z_{1}>0\right\}$. It is clear that $\partial \omega$ is admissible and has two characteristic points.

4.7. Proposition. If $M=\partial D$ is strictly pseudoconvex, then $M$ has a local neighborhood basis of open sets $\omega$ of the type described above, such that $\partial \omega$ is admissible and has precisely two characteristic points. 
Proof. In view of the above example, the proof is clear if $M$ is strictly convex. If $M$ is strictly pseudoconvex, then, locally, there is an ambient biholomorphism which takes $M$ to a strictly convex hypersurface. Since a biholomorphism takes characteristic points to characteristic points, the proof is now complete. Q.E.D.

4.8. Proposition. Suppose $\omega \subset M$ is defined as above and suppose $\partial \omega$ has only two characteristic points. Then $\partial \omega$ is admissible.

Proof. The proof will be clear once we establish that $z_{0} \in \partial \omega$ is a characteristic point if and only if $z_{0}$ is a critical point for $\left.\operatorname{Im}(h)\right|_{\partial \omega}$. Suppose $\rho$ is the defining function for $M$. Recall that $r=\operatorname{Re}(h)$ is the defining function for $\partial \omega$. Now $z_{0}$ is a characteristic point if and only if $\nabla r\left(z_{0}\right)=C J \nabla \rho\left(z_{0}\right)$, where $C$ is a nonzero real number. Since $h$ is holomorphic, the Cauchy-Riemann equations imply that $\nabla(\operatorname{Im} h)=-J(\nabla r)$. Therefore, $z_{0}$ is a characteristic point if and only if $\nabla(\operatorname{Im} h)\left(z_{0}\right)$ $=C(\nabla \rho)\left(z_{0}\right)$. Now if $X_{z_{0}}$ is a tangent vector to $\partial \omega$ at $z_{0}$, then $X_{z_{0}}$ is also a tangent vector to $M$ at $z_{0}$, and so $\left\langle\nabla \rho\left(z_{0}\right), X_{z_{0}}\right\rangle=0$. Therefore, $z_{0}$ is a characteristic point if and only if $\left\langle\nabla(\operatorname{Im} h)\left(z_{0}\right), X_{z_{0}}\right\rangle=0$ for each tangent vector $X_{z_{0}}$ to $\partial \omega$ at $z_{0}$. Q.E.D.

We now construct a regular generating function, $v(\zeta, z)$, near $\partial \omega \times\{\partial \omega-C\}$. Using Lemma 2.5 from Henkin [7], there exists a holomorphic mapping: $v(\zeta, z)$ : $\bar{D} \times \bar{D} \rightarrow C^{n}$ which is defined near $\bar{D} \times \bar{D}$ such that $v(\zeta, z) \cdot(\zeta-z)=h(\zeta)-$ $h(z)$. If $D$ is actually convex, then this construction is easy, for we have

$$
\begin{aligned}
h(\zeta)-h(z) & =\int_{0}^{1} \frac{d}{d t}\{h(t \zeta+(1-t) z)\} d t \\
& =\sum_{j=1}^{n}\left(\int_{0}^{1} \frac{\partial h}{\partial z_{j}}(t \zeta+(1-t) z) d t\right)\left(\zeta_{j}-z_{j}\right),
\end{aligned}
$$

and we may let

$$
v_{j}(\zeta, z)=\int_{0}^{1} \frac{\partial h}{\partial z_{j}}(t \zeta+(1-t) z) d t .
$$

The singular set for the kernels constructed in $\$ 4.1$ becomes $B=\{(\zeta, z) \in M \times$ $M ; h(\zeta)=h(z)\}$. If $z$ is fixed on $M$, then the $\zeta$-singular set for the kernels is the set $B_{z}=\{\zeta \in M ; h(\zeta)=h(z)\}$.

4.9. LEMMA. (a) If $\partial \omega$ is admissible, then there is a neighborhood $V=U_{1} \times U_{2}$ in $M \times M$ which contains $\{\partial \omega\} \times\{\partial \omega-C\}$ such that $v(\zeta, z)$ is a regular generating function for $V$. Furthermore, if $z \in \omega$ then $B_{z} \subset \subset \omega$ and if $z \in \omega^{c}$ (but near $\partial \omega$ ) then $B_{z} \subset \subset \omega^{c}$.

(b) There exists a neighborhood $V^{\prime}=U_{1}^{\prime} \times U_{2}^{\prime}$ in $M \times M$ which contains $\{\partial \omega-$ $C\} \times\{\partial \omega\}$, such that $\left.v\right|_{V^{\prime}}$ is a regular generating function for $V^{\prime}$.

REMARK. Note that we are not assuming that $\partial \omega$ is admissible in part (b).

Proof. It suffices to check Definition 4.1 holds for $(\zeta, z) \in\{\partial \omega\} \times\{\partial \omega-C\}$ if $\partial \omega$ is admissible and for $(\zeta, z) \in\{\partial \omega-C\} \times\{\partial \omega\}$. Now, it is clear that $\left.\nabla_{\zeta}\left\{\operatorname{Re} v\left(\zeta, z_{0}\right) \cdot\left(\zeta-z_{0}\right)\right\}\right|_{\zeta=z_{0}}=(\nabla r)\left(z_{0}\right)$. The Cauchy-Riemann equations imply that $\left.\nabla_{\zeta}\left\{\operatorname{Im} v\left(\zeta, z_{0}\right) \cdot\left(\zeta-z_{0}\right)\right\}\right|_{\zeta=z_{0}}=-J(\nabla r)\left(z_{0}\right)$. If $\left(z_{0}, z_{0}\right) \in\{\partial \omega\} \times\{\partial \omega-C\}$, 
then $z_{0}$ is noncharacteristic and the matrix in part (a) of Definition 4.1 has maximal rank.

Now suppose $\left(\zeta_{0}, z_{0}\right) \in V$ with $\zeta_{0} \in B_{z_{0}}$. Since $\partial \omega$ is admissible, $\zeta_{0}$ is not a characteristic point. Therefore, the matrix in part (b) of Definition 4.1 has maximal rank.

Note that $B_{z} \subset\{\zeta \in M ; r(\zeta)=r(z)\}$ which is a compact set if $z \in \omega$ or if $z \in \omega^{c}$ near $\partial \omega$.

The proof of part (b) is identical to the proof of part (a). Since $\zeta_{0} \notin C$ by hypothesis, it is unnecessary to assume $\partial \omega$ is admissible. Q.E.D.

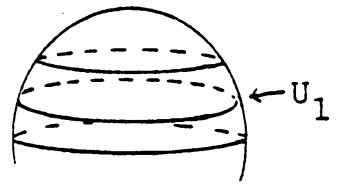

S-space $\mathrm{p}_{1}, \mathrm{p}_{2}$

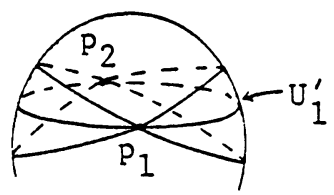

5 - space

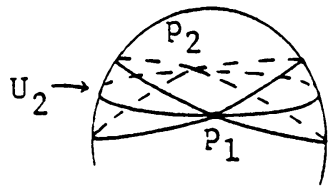

z-space

are characteristic points

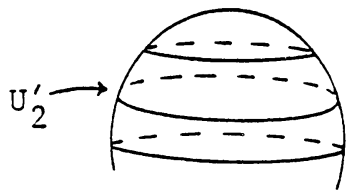

z-space

From Theorem 4.3 the principal value limits of $E_{123}, E_{13}, E_{23}$ exist on $V=U_{1} \times$ $U_{2}$ and $V^{\prime}=U_{1}^{\prime} \times U_{2}^{\prime}$ and define regular kernels. Now, $v(\zeta, z)$ is holomorphic in both $\zeta$ and $z ; u(\zeta, z)$ is holomorphic in $z$; and $t u(\zeta, z)$ is holomorphic in $\zeta$. Therefore, using Proposition 2.23 the $E_{13}$ and $E_{23}$ kernels become:

$$
\begin{aligned}
& E_{13}(\zeta, z)=(2 \pi i)^{-n} \omega_{1} \wedge \omega_{3}\left[\frac{\bar{\partial}_{\zeta} u \cdot d(\zeta, z)}{u \cdot(\zeta-z)}\right]^{n-2}, \\
& E_{23}(\zeta, z)=(2 \pi i)^{-n} \omega_{2} \wedge \omega_{3}\left[\frac{\bar{\partial}_{z}{ }^{t} u \cdot d(\zeta-z)}{{ }^{t} u \cdot(\zeta-z)}\right]^{n-2} .
\end{aligned}
$$

In particular, $E_{13}(\zeta, z)$ has degree $n-2$ in $d \bar{\zeta}$ and $E_{23}(\zeta, z)$ has degree zero in $d \bar{\zeta}$. Therefore, in view of the current pairing on $M$, we have the following proposition.

4.10. Proposition. Suppose $g \in \mathscr{D}_{M}^{p, q}\left(U_{1}\right)$ or $g \in \mathscr{Q}_{M}^{p, q}\left(U_{1}^{\prime}\right)$; then $E_{13}(g)=0$ unless $q=1$ and $E_{23}(g)=0$ unless $q=n-1$.

We also need to extend these kernels to operate on currents in $\mathcal{E}_{M}^{\prime *}\left(U_{1}\right)$ which are smooth outside $C$. Let $E$ be any one of the kernels $E_{123}, E_{13}, E_{23}$ or $R$.

4.11. Lemma. Let $\partial \omega$ be admissible. Suppose $g \in \mathcal{E}_{M}^{p, q}\left(U_{1}\right)$ is smooth on $U_{1}-C$; then $E(g)$ is smooth on $U_{2}$. 
Proof. Fix $z_{0} \in U_{2}$. $B_{z_{0}} \cap C=\varnothing$ because $\partial \omega$ is admissible. Therefore there exists $\eta \in \mathscr{D}\left(U_{1}\right)$ with $\eta \equiv 1$ near $C$, such that supp $\eta \cap B_{z}=\varnothing$ for each $z$ near $z_{0}$. Therefore, $E(\eta g)(z)$ is smooth for $z$ near $z_{0}$. Since $(1-\eta) g \in \mathscr{P}_{M}^{p, q}\left(U_{1}\right)$, Theorem 4.3 implies that $E((1-\eta) g)$ is smooth on $U_{2}$ and the lemma follows. Q.E.D.

The next theorem is the first of three main theorems in this section. It enables us to solve the $\bar{\partial}_{M}$ equation smoothly up to the boundary except at characteristic points.

4.12. TheOrem. Suppose $M=\partial D$ is the boundary of a strictly pseudoconvex domain. Suppose $\omega \subset \subset M$ is defined by $\omega=\{z \in M ; \operatorname{Re} h(z)<0\}$ where $h$ is holomorphic near $\bar{D}$ and suppose $\partial \omega$ is admissible. Suppose $f \in \mathcal{E}_{M}^{p, q}(\omega), 1<q<n-$ 3, has a distribution extension $\tilde{f}$ to a neighborhood of $\bar{\omega}$ in $M$, which is smooth on $\bar{\omega}-C$. If $\bar{\partial}_{M} f \equiv 0$ on $\omega$, then $u=E_{123}\left(\bar{\partial}_{M} \tilde{f}\right)+R(\tilde{f})$ is smooth on $\bar{\omega}-C$ and satisfies $\bar{\partial}_{M} u=f$ on $\omega$.

Remark. By Proposition 4.8, Theorem 4.12 holds if $\partial \omega$ has two characteristic points. Note also that the hypothesis on $f$ is satisfied if $f$ is smooth on $\bar{\omega}$.

Proof. Let $\tilde{f} \in \mathcal{E}_{M}^{\prime p, q}\left(U_{1}\right)$ be an extension of $f$ which is smooth on $U_{1}-C$. We claim

$$
u(z)=E_{123}\left(\bar{\partial}_{M} \tilde{f}\right)(z)+R(\tilde{f})(z)
$$

is smooth on $\omega \cup U_{2}$ (so in particular, $u$ is smooth on $\bar{\omega}-C$ ). From Lemma 4.11, $u$ is smooth on $U_{2}$. Now, $\operatorname{supp}\left\{\bar{\partial}_{M} \tilde{f}\right\} \subset \omega^{c}$ because $\bar{\partial}_{M} f=0$ on $\omega$. By Lemma 4.9 part (a), we have that $B_{z} \cap \operatorname{supp} \bar{\partial}_{M} \tilde{f}=\varnothing$ for each $z \in \omega$. Therefore $E_{123}\left(\bar{\partial}_{M} \tilde{f}\right)$ is also smooth on $\omega$.

Next, we check that $\bar{\partial}_{M} u=f$ on $\omega$. Since $B \cap\left\{\operatorname{supp} \bar{\partial}_{M} \tilde{f}\right\} \times\{\omega\}=\varnothing$, equation (4.2) is valid on $\left\{\operatorname{supp} \bar{\partial}_{M} f\right\} \times\{\omega\}$. Thus, we have

$$
\begin{aligned}
\bar{\partial}_{M} u & =\bar{\partial}_{M}\left\{E_{123}\left(\bar{\partial}_{M} \tilde{f}\right)\right\}+\bar{\partial}_{M}\{R(\tilde{f})\} \\
& =-\left(\bar{\partial}_{M} E_{123}\right)\left(\bar{\partial}_{M} \tilde{f}\right)+\tilde{f}-R\left(\bar{\partial}_{M} \tilde{f}\right) \\
& =\left(E_{23}-E_{13}+R\right)\left(\bar{\partial}_{M} \tilde{f}\right)+\tilde{f}-R\left(\bar{\partial}_{M} \tilde{f}\right)
\end{aligned}
$$

on $\omega$. We have used Theorem 3.9 for the $R$ kernel and we have used the operator equation $-\left[\bar{\partial}_{M} E_{123}\right]=\bar{\partial}_{M} \circ E_{123}-E_{123} \circ \bar{\partial}_{M}$ (see Theorem 2.8 in [6]). Note that $\left(L_{M}-{ }^{t} L_{M}\right)(\tilde{f}) \equiv 0$ by type considerations. By Proposition $4.10, E_{13}\left(\bar{\partial}_{M} \tilde{f}\right)=$ $E_{23}\left(\bar{\partial}_{M} \tilde{f}\right)=0$ because $\bar{\partial}_{M} \tilde{f} \in \mathcal{E}_{M}^{\prime p, q+1}\left(U_{1}\right)$ and $2<q+1<n-2$. So, we are left with $\bar{\partial}_{M} u=\tilde{f}=f$ on $\omega$. Q.E.D.

REMARKs. 1. The only place Theorem 4.3 is used in the proof of Theorem 4.12 is to prove that $u$ is smooth on $\bar{\omega}-C$. The next main theorem will use Theorem 4.3 in a much stronger way.

2. If $f \in \mathcal{E}_{M}^{p, q}(\bar{\omega})$, then the solution given in Theorem 4.12 is $u=E_{123}\left(\bar{\partial}_{M} \tilde{f}\right)+$ $R(\tilde{f})$ which is valid for any smooth extension $\tilde{f} \in \mathscr{Q}_{M}^{p, q}\left(\omega \cup U_{1}\right)$. It is suggestive to try a particular nonsmooth extension $\tilde{f}=\chi_{\omega} f$. Then $\bar{\partial}_{M} \tilde{f}=[\partial \omega]^{0,1} \wedge f$ and the solution becomes

$$
u(z)=\int_{\zeta \in \partial \omega} E_{123}(\zeta, z) \wedge f(\zeta)+\int_{\zeta \in \omega} R(\zeta, z) \wedge f(\zeta)
$$


It is easy to see that $u(z)$ is smooth on $\omega$ (interior regularity) without the use of Theorem 4.3. The same calculation in the proof of Theorem 4.12 shows that $\bar{\partial}_{M} u=f$ on $\omega$. In the case where $M$ is strictly convex and the defining function $h$ is complex linear, this solution was discovered by Henkin [10]. In Chapter 5, we shall prove that the Henkin solution is tangentially smooth up to $\partial \omega-C$. However, we know nothing about the continuity of normal derivatives of the Henkin solution at the boundary of $\omega$.

Since $v$ is also a regular generating function for $V^{\prime}=U_{1}^{\prime} \times U_{2}^{\prime}$, the kernels $E_{123}$, $E_{13}, E_{23}$ and $S$ are defined and regular on $V^{\prime}$. We may assume that $U_{2}^{\prime}=\{z \in M$; $|r(z)|<\delta\}$ for some $\delta>0$ and that $U_{1}^{\prime} \subset U_{2}^{\prime}$.

4.13. Lemma. Suppose $g \in \mathcal{E}_{M}^{p, q}(\omega)$ with $g \equiv 0$ near $\omega-U_{1}^{\prime}$, and near each characteristic point on $\partial \omega$. Then the $S$ kernel extends to operate on $g$ and $S(g)$ is smooth on $\omega$.

Proof. For $t>0$, let $\eta_{t} \in \mathscr{D}(\omega)$ with $\eta_{t} \equiv 1$ on $\{z \in \omega ; r(z)<-t\}$. Since $g \equiv 0$ near $\omega-U_{1}^{\prime}, \eta_{t} g \in \mathscr{D}_{M}^{p, q}\left(U_{1}^{\prime}\right)$ for each $t>0$. Hence $S\left(\eta_{t} g\right)$ is smooth on $U_{2}^{\prime}$ by Theorem 4.3. If $t<s$, then $S\left(\left(\eta_{t}-\eta_{s}\right) g\right)(z)=0$ for $z \in U_{2}^{\prime}$ with $r(z)<-s$ because supp $S \subset B \subset\{(\zeta, z) \in M \times M ; r(\zeta)=r(z)\}$. Therefore, we may define $S(g)=\lim _{t \rightarrow 0^{+}} S\left(\eta_{t} g\right)$ on $U_{2}^{\prime}$. Since $g$ vanishes on $\{z \in \omega ; r(z)<-\delta\}$, it is clear that we may define $S(g) \equiv 0$ on $\omega-U_{2}^{\prime}$. Q.E.D.

The next theorem is the second main theorem in this section. It enables us to solve the equation $\bar{\partial}_{M} u=f$ with certain no growth restrictions at the boundary.

4.14. TheOREM. Suppose $f \in \mathcal{E}_{M}^{p, q}(\omega), 2<q<n-3$, and suppose $f$ vanishes near each characteristic point on $\partial \omega$. If $\bar{\partial}_{M} f=0$ on $\omega$, then there exists $u \in \mathcal{E}_{M}^{p, q-1}(\omega)$ with $\bar{\partial}_{M} u=f$ on $\omega$.

Proof. Since $\omega^{c} \cap U_{1}^{\prime}$ and $\omega-U_{1}^{\prime}$ are disjoint sets which are closed relative to $\omega \cup U_{1}^{\prime}$, there exists $\eta \in \mathcal{E}\left(\omega \cup U_{1}^{\prime}\right)$ with $\eta \equiv 0$ near $\omega-U_{1}^{\prime}$ and $\eta \equiv 1$ near $\omega^{c} \cap U_{1}^{\prime}$. We can also require $\eta(z)=0$ for $r(z)<-\delta / 2$. It is clear that $\eta$ does not have a continuous extension at a characteristic point. Consider

$$
u=R((1-\eta) f)+S(\eta f)-E_{123}\left(\bar{\partial}_{M} \eta \wedge f\right) .
$$

We claim that $u$ is smooth on $\omega$ and solves $\bar{\partial}_{M} u=f$ on $\omega$. Since $f$ vanishes near each characteristic point on $\partial \omega, \eta f \equiv 0$ near $\omega-U_{1}^{\prime}$ and near each characteristic point. Therefore $S(\eta f)$ is smooth on $\omega$ by Lemma 4.13. $R((1-\eta) f)$ is smooth on $M$ because $(1-\eta) f$ is smooth with compact support in $\omega$. Since $\bar{\partial}_{M} \eta \wedge f \in$ $\mathcal{Q}_{M}^{p, q+1}\left(U_{1}^{\prime}\right), E_{123}\left(\bar{\partial}_{M} \eta \wedge f\right)$ is smooth on $U_{2}^{\prime}$ by Theorem 4.3. $E_{123}\left(\bar{\partial}_{M} \eta \wedge f\right)$ is also smooth on $\omega-U_{2}^{\prime}=\{z \in \omega ; r(z) \leqslant-\delta\}$ because $\bar{\partial}_{M} \eta \wedge f(z)=0$ for $r(z)<$ $-\delta / 2$. Therefore $u$ is smooth on $\omega$.

Using Theorem 3.9 for the $R$ kernel and Theorem 4.3, and the fact that $\bar{\partial}_{M}\left\{\bar{\partial}_{M} \eta \wedge f\right\}=0$, we have

$$
\begin{aligned}
\bar{\partial}_{M} u= & (1-\eta) f+R\left(\bar{\partial}_{M} \eta \wedge f\right)+\bar{\partial}_{M}\{S(\eta f)\} \\
& +\left(E_{13}-E_{23}-R\right)\left(\bar{\partial}_{M} \eta \wedge f\right)+S\left(\bar{\partial}_{M} \eta \wedge f\right)
\end{aligned}
$$


Since $\bar{\partial}_{M} \eta \wedge f \in \mathscr{D}_{M}^{p, q+1}\left(U_{1}^{\prime}\right)$ and $3 \leqslant q+1 \leqslant n-2,\left(E_{13}-E_{23}\right)\left(\bar{\partial}_{M} \eta \wedge f\right)=0$ by Proposition 4.10. So, we have

$$
\bar{\partial}_{M} u=(1-\eta) f+\bar{\partial}_{M}\{S(\eta f)\}+S\left(\bar{\partial}_{M} \eta \wedge f\right) \text { on } \omega \cup U_{2}^{\prime}
$$

Since $\bar{\partial}_{M} f=0$, equation (4.5) of Theorem 4.3 yields:

$$
\bar{\partial}_{M}\{S(\eta f)\}+S\left(\bar{\partial}_{M} \eta \wedge f\right)=-\left(\bar{\partial}_{M} S\right)(\eta f)=\eta f \text { on } \omega \text {. }
$$

Note again that the $E_{13}, E_{23}, L_{M}$ and ' $L_{M}$ terms disappear by type considerations. Therefore, we have $\bar{\partial}_{M} u=f$ on $\omega$ as desired. Q.E.D.

Remark. Note that we did not assume that $\partial \omega$ is admissible in Theorem 4.14.

The next theorem is the third main theorem in this section and enables us to solve $\bar{\partial}_{M} u=f$ with a compactly supported solution in $\omega$, if $f$ has compact support in $\omega$. This theorem does not use Theorem 4.3.

4.15. Theorem. Suppose $f \in \mathscr{D}_{M}^{p, q}(\omega)$ with $2 \leqslant q \leqslant n-2$, and suppose $\bar{\partial}_{M} f \equiv 0$. Then there exists $u \in \mathscr{D}_{M}^{P, q}(\omega)$ with $\bar{\partial}_{M} u=f$.

Proof. Assume that supp $f \subset\{z \in \omega ; r(z)<-\delta\}$, for some $\delta>0$. Choose $\eta \in \mathcal{E}(M)$ with $\eta(z)=0$ if $r(z) \leqslant-\delta$ and $\eta(z)=1$ if $r(z) \geqslant-\delta / 2$. Consider

$$
u(z)=R(f)(z)+\left[\bar{\partial}_{M}\left(\eta(z) E_{123}\right)\right](f)(z)
$$

where $\bar{\partial}_{M}$ on the right is taken on the product space, $M \times M$. Since $\bar{\partial}_{M} f \equiv 0$, we see that $u=R(f)-\bar{\partial}_{M}\left\{\eta E_{123}(f)\right\}$. Note that $u$ is smooth on $M$ because if $z \in \operatorname{supp} \eta$, then $B_{z} \cap \operatorname{supp} f=\varnothing$. Clearly, we have $\bar{\partial}_{M} u=f$ because $\bar{\partial}_{M}\{R(f)\}=$ $f$. It remains to check that $u$ has compact support in $\omega$. Expanding (4.16), we have

$$
\begin{aligned}
u & =R(f)+\left(\bar{\partial}_{M} \eta\right) \wedge E_{123}(f)+\eta\left(\bar{\partial}_{M} E_{123}\right)(f) \\
& =(1-\eta) R(f)+\left(\bar{\partial}_{M} \eta\right) \wedge E_{123}(f) .
\end{aligned}
$$

We have used (4.2) and Proposition 4.10. Since $1-\eta$ and $\bar{\partial}_{M} \eta$ have compact support in $\omega$, clearly $u$ has compact support in $\omega$. Q.E.D.

REMARK. Theorem 4.15 is definitely false for $q=1$. If it were true, then, together with the uniqueness theorems for C-R functions proved in [13], one could prove an extension theorem for C-R functions analogous to the Bochner-Hartogs extension theorem for holomorphic functions. (See the proof of Theorem 2.3.2 in Hörmander [12].) Such a theorem does not hold for C-R functions. For example, let $M$ be the unit sphere in $\mathbf{C}^{n}, \omega=\left\{z \in M ; \operatorname{Re} z_{1}>0\right\}$, and let $Q$ be the annular region defined by $Q=\left\{z \in M ; 0<\operatorname{Re} z_{1}<\frac{1}{2}\right\}$. The set $\tilde{Q}=\left\{z \in \mathbf{C}^{n} ; 0<\operatorname{Re} z_{1}<\frac{1}{2}\right\}$ is a convex set and hence a domain of holomorphy. So there exists a holomorphic function $f$, which does not extend across any part of the boundary of $\tilde{Q}$. Hence, $\left.f\right|_{Q}$ is a $\mathrm{C}-\mathrm{R}$ function on $C$ which has no $\mathrm{C}-\mathrm{R}$ extension to all of $\omega$.

4.3. Technical proofs. We first prove Theorem 3.8 on the regularity for the $R$ kernel.

Proof of Theorem 3.8. By analyzing the pieces of the $R$ kernel, we need to show that if $f \in \mathscr{D}_{M}^{n, n-1}(M)$, then 


$$
(K f)(z)=\int_{\zeta \in M} \frac{g(\zeta, z) f(\zeta)}{(u \cdot(\zeta-z))^{p}\left({ }^{t} u \cdot(\zeta-z)\right)^{q}}
$$

is smooth in $z \in M$, where $p+q=n$ and $g(\zeta, z)$ is smooth with $g(\zeta, z)=$ $\vartheta(|\zeta-z|)$. The estimate on $g$ follows because $u(z, z)={ }^{t} u(z, z)$, and hence

$$
u(\zeta, z) \cdot d(\zeta-z) \wedge^{t} u(\zeta, z) \cdot d(\zeta-z)=\theta(|\zeta-z|)
$$

To prove the $R$ kernel is regular, we must show that for each $\left(\zeta_{0}, z_{0}\right) \in M \times M$, there exists an open set $U_{1} \times U_{2}$ in $M \times M$ containing $\left(\zeta_{0}, z_{0}\right)$, such that if $f \in \mathscr{D}_{M}^{n, n-1}\left(U_{1}\right)$, then $(K f)(z)$ is smooth for $z \in U_{2}$. The theorem then follows from a partition of unity argument.

Since $u$ is a strong support function for $M$ (Definition 3.4), $u \cdot(\zeta-z)$ and ${ }^{t} u \cdot(\zeta-z)$ are nonvanishing for $(\zeta, z) \in M \times M$ and $\zeta \neq z$. Therefore, if $\zeta_{0} \neq z_{0}$, then we can choose $U_{1} \times U_{2}$ such that $U_{1} \times U_{2} \cap \Delta=\varnothing$. So we may assume $\zeta_{0}=z_{0}$.

From Lemma 3.7 part $(\mathrm{b}), u \cdot(\zeta-z)=h \hat{u} \cdot(\zeta-z)$ for $(\zeta, z)$ near $\Delta$ where

$$
\hat{u}_{j}(\zeta, z)=\frac{\partial \rho}{\partial \zeta_{j}}(\zeta)-\frac{1}{2} \sum_{k=1}^{n} \frac{\partial^{2} \rho}{\partial \zeta_{j} \partial \zeta_{k}}(\zeta)\left(\zeta_{k}-z_{k}\right)
$$

and $h$ is a nonzero smooth function. Since we will be working near $\Delta$, we may replace $u$ by $\hat{u}$ in the definition of the $R$ kernel.

We must make a change of coordinates given in the next lemma.

4.17. LEMMA. There exist a neighborhood $\tilde{U}$ in $\mathbf{C}^{n}$ containing $z_{0}$ and $\eta>0$ and $a$ smooth map $\Psi: \tilde{U} \times \tilde{U} \rightarrow \mathbf{C}^{n}$ with the following properties:

(a) For fixed $z \in \tilde{U}, \Psi_{z}(\zeta)=\Psi(\zeta, z): \Psi_{z}^{-1}\{B(0, \eta)\} \rightarrow B(0, \eta)$ is a diffeomorphism, where $B(0, \eta)$ is the ball in $\mathbf{C}^{n}$ of radius $\eta$.

(b) If we write $\Psi_{z}(\zeta)=\left(w_{1}(\zeta, z), \ldots, w_{n}(\zeta, z)\right)$, then $\operatorname{Re} w_{1}(\zeta, z)=\rho(\zeta)$, $\operatorname{Im} w_{1}(\zeta, z)=\operatorname{Im}\{\hat{u}(\zeta, z) \cdot(\zeta-z)\}$, and $\Psi_{z}(z)=0$ for $z \in M$.

(c) $\Psi_{z}^{-1}(w)$ is smooth in both $z \in \tilde{U}$ and $w \in B(0, \eta)$.

Proof. We note that

$$
\left.\nabla_{\zeta}\left\{\operatorname{Im} \hat{u}\left(\zeta, z_{0}\right) \cdot\left(\zeta-z_{0}\right)\right\}\right|_{\zeta=z_{0}}=-\frac{1}{2} J(\nabla \rho)\left(z_{0}\right)
$$

where $J$ is the linear mapping induced by muliplication by $i$. Therefore, the real Jacobian of the map

$$
\zeta \mapsto\left(\begin{array}{l}
\rho(\zeta) \\
\operatorname{Im} \hat{u}\left(\zeta, z_{0}\right) \cdot\left(\zeta-z_{0}\right)
\end{array}\right) \in \mathbf{R}^{2}
$$

at $\zeta=z_{0}$ has maximal rank and we can let $w_{j}(\zeta, z)=w_{j} \cdot(\zeta-z), j>2$, for some suitable choice of vectors $w_{j} \in \mathbf{C}^{n}$. The rest of the lemma follows from the implicit function theorem. Q.E.D.

From Lemma 4.17, we have $\Psi_{z}^{-1}\left\{w \in C^{n} ; \operatorname{Re} w_{1}=0\right\} \subset M$, and $\Psi_{z}^{-1}(0)-z=$ 0 for $z \in M \cap \tilde{U}$. A Taylor series expansion in $w$ about the origin yields

$$
\Psi_{z}^{-1}(w)-z \approx|w| \text { for } z \in \tilde{U} \cap M \text { and }|w|<\eta \text { and } \operatorname{Re} w_{1}=0 .
$$


For each $z \in \tilde{U} \cap M$, we pull back the integral in $(K f)(z)$ to obtain

$$
(K f)(z)=\int_{w \in \mathbf{R}^{2 n-1}} \frac{g_{1}(w, z) f_{1}(w, z) d \lambda(w)}{\left(u_{1}(w, z)\right)^{p}\left({ }^{t} u_{1}(w, z)\right)^{q}}
$$

where $d \lambda(w)$ denotes Lebesgue measure in $\mathbf{R}^{2 n-1}$ and

$$
\begin{aligned}
f_{1}(w, z) d \lambda(w) & =\left(\Psi_{z}^{-1 *}\{f\}\right)(w), \\
g_{1}(w, z) & =g\left(\Psi_{z}^{-1}(w), z\right), \\
u_{1}(w, z) & =\hat{u}\left(\Psi_{z}^{-1}(w), z\right) \cdot\left(\Psi_{z}^{-1}(w)-z\right), \\
{ }^{t} u_{1}(w, z) & ={ }^{t} \hat{u}\left(\Psi_{z}^{-1}(w), z\right) \cdot\left(\Psi_{z}^{-1}(w)-z\right) .
\end{aligned}
$$

From the choice of $\Psi$, we see that $u_{1}(w, z)=\operatorname{Re} u_{1}(w, z)+i y_{1}$ where $y_{1}=\operatorname{Im} w_{1}$.

4.19. LeMMA. There exists a neighborhood $U_{2} \subset M$ containing $z_{0}$ and $\eta>0$ such that the following estimates hold uniformly for $z \in U_{2}$ and $|w|<\eta$ with $\operatorname{Re} w_{1}=0$.

(a) $g_{1}(w, z)=\theta(|w|)$.

(b) $u_{1}(w, z) \approx\left(|w|^{2}+\left|y_{1}\right|\right)$ where $y_{1}=\operatorname{Im} w_{1},{ }^{t} u_{1}(w, z) \approx\left(|w|^{2}+\left|y_{1}\right|\right), u_{1}(w, z)$ $-{ }^{\prime} u_{1}(w, z)=\vartheta\left(|w|^{2}\right)$.

(c) If $X_{z}$ is a vector field in $z$ which is tangential to $M$, then

$$
\begin{aligned}
X_{z} g_{1}(w, z) & =\Theta(|w|), \\
X_{z} u_{1}(w, z) & =\Theta\left(|w|^{2}\right), \\
X_{z}{ }^{t} u_{1}(w, z) & =\Theta\left(|w|^{2}\right) .
\end{aligned}
$$

Proof. From the estimate in (4.18), we have

$$
g_{1}(w, z)=g\left(\Psi_{z}^{-1}(w), z\right)=\mathcal{O}\left(\left|\Psi_{z}^{-1}(w)-z\right|\right)=\mathcal{O}(|w|) .
$$

In part (b), the estimate on $u_{1}(w, z)$ will follow if we can show that $\operatorname{Re} u_{1}(w, z) \approx$ $|w|^{2}$ for $\operatorname{Re} w_{1}=0$ and $|w| \leqslant \eta$ and $z \in U_{2}$. Since $u$ is a strong support function, there exists an open set $U$ in $M$ containing $z_{0}$ such that

$$
\operatorname{Re}\{\hat{u}(\zeta, z) \cdot(\zeta-z)\} \approx|\zeta-z|^{2} \text { for }(\zeta, z) \in U \times U .
$$

Therefore, the estimate on $\operatorname{Re} u_{1}$ will follow if we choose $\eta>0$ and $U_{2}$ such that for each $z \in U_{2}, \Psi_{z}^{-1}\{B(0, \eta)\} \subset U$. (Here $B(0, \eta)$ is the ball of radius $\eta$ in $\mathbf{R}^{2 n-1}$.)

Since $\hat{u}(z, z)={ }^{t} \hat{u}(z, z)$

$$
\hat{u}(\zeta, z) \cdot(\zeta-z)-{ }^{t} \hat{u}(\zeta, z) \cdot(\zeta-z)=\vartheta\left(|\zeta-z|^{2}\right) .
$$

Therefore, the estimate on $u_{1}-{ }^{t} u_{1}$ follows from (4.18).

To prove the estimate on ${ }^{t} u_{1}$, we need the following estimates

$$
\begin{aligned}
& \operatorname{Re}{ }^{t} u_{1}(w, z) \gtrsim|w|^{2} \quad \text { for } z \in U_{1},|w|<\eta \text { and } \operatorname{Re} w_{1}=0, \\
& \left|\operatorname{Im}{ }^{\prime} u_{1}(w, z)\right| \gtrsim\left|y_{1}\right|-|w|^{2} \text { for } z \in U_{1},|w|<\eta \text { and } \operatorname{Re} w_{1}=0 .
\end{aligned}
$$

The estimate on $\operatorname{Re}{ }^{t} u_{1}$ follows because $u$ is a strong support function. For $\operatorname{Im}{ }^{\prime} u_{1}$, we have

$$
\begin{aligned}
|\operatorname{Im} u| & =\left|\left(\operatorname{Im}{ }^{t} u-\operatorname{Im} u\right)+\operatorname{Im} u\right| \\
& =\left|\mathcal{O}\left(|w|^{2}\right)+y_{1}\right| \gtrsim\left|y_{1}\right|-|w|^{2}
\end{aligned}
$$


Now, from (4.20)

$$
\begin{aligned}
\left.\right|^{t} u(w, z) \mid & \geqslant \max \left\{\left|\operatorname{Re}{ }^{t} u\right|,\left|\operatorname{Im}{ }^{t} u\right|\right\} \\
& \gtrsim \max \left\{|w|^{2},\left|y_{1}\right|-|w|^{2}\right\} \gtrsim|w|^{2}+\left|y_{1}\right|
\end{aligned}
$$

where the last inequality follows from the following inequality used in GrauertLieb [5, p. 36]:

If $\alpha, \beta, \gamma$ are positive real numbers, then

$$
\max \{\alpha, \beta-\gamma\} \geqslant(2+\gamma / \alpha)^{-1}(\alpha+\beta) \text {. }
$$

To prove part (c), we first note that if $X_{z}$ is a vector field in $z$, and if $h(w, z)=\mathcal{\theta}\left(|w|^{k}\right)$, then $X_{z} h(w, z)=\vartheta\left(|w|^{k}\right)$. (See the notation at the beginning of Chapter 3.) Therefore, if $X_{z}$ is tangential to $M$, the estimates on $X_{z} g_{1}$ and $X_{z} u_{1}$ follow easily, because $g_{1}(w, z)=\mathcal{O}(|w|), \operatorname{Re} u_{1}(w, z)=\vartheta\left(|w|^{2}\right)$, and $\operatorname{Im} u_{1}=y_{1}$ for $z \in M$.

We also have

$$
X_{z}{ }^{t} u_{1}=X_{z}\left\{{ }^{t} u_{1}-u_{1}\right\}+X_{z}\left\{u_{1}\right\}=\theta\left(|w|^{2}\right)
$$

because ${ }^{t} u_{1}-u_{1}=\theta\left(|w|^{2}\right)$. Q.E.D.

REMARK. We emphasize that the estimates in parts (a) and (b) only hold for $z \in M$ near $z_{0}$, because (4.18) only holds for $z \in M$. Hence, we only get the estimates in part (c) for vector fields which are tangential to $M$.

Choose $\eta>0$ to satisfy Lemmas 4.19 and 4.17 and choose $U_{2} \subset M$ to satisfy Lemma 4.19. By shrinking $U_{2}$ if necessary, there exists an open set $U_{1} \subset M$ containing $z_{0}$ such that $\Psi\left\{U_{1} \times U_{2}\right\} \subset B(0, \eta)$. From now on, we shall require $f$ to have compact support in $U_{1}$. Thus, for each $z \in U_{2}$, the $w$-support of $f_{1}(w, z)$ is contained in $B(0, \eta)$.

Let $I(w, z)$ be the integrand appearing in $(K f)(z)$. From Lemma 4.19, we have

$$
|I(w, z)| \lesssim \frac{|w|}{\left(|w|^{2}+\left|y_{1}\right|\right)^{n}} \leqslant \frac{1}{\left(|w|^{2}+\left|y_{1}\right|\right)^{n-1 / 2}}
$$

This estimate holds uniformly for $|w| \leqslant \eta$ with $\operatorname{Re} w_{1}=0$ and $z \in U_{2}$.

If $X_{z}$ is a vector field which is tangential to $M$, then Lemma 4.19 implies

$$
\begin{aligned}
\left|X_{z}\left(u_{1}(w, z)\right)^{-p}\right| & =\left|\left(X_{z} u_{1}(w, z)\right) \cdot\left(u_{1}(w, z)\right)^{-(p+1)}\right| \\
& \lesssim|w|^{2}\left(|w|^{2}+\left|y_{1}\right|\right)^{-(p+1)} \lesssim\left(|w|^{2}+\left|y_{1}\right|\right)^{-p} .
\end{aligned}
$$

Similarly, we have $\left|X_{z}\left({ }^{t} u_{1}\right)^{-q}\right| \lesssim\left(|w|^{2}+\left|y_{1}\right|\right)^{-q}$. Thus, the estimates are no worse for $X_{z} I(w, z)$ than they are for $I(w, z)$. If $X_{1_{z}} \ldots X_{N_{z}}$ are vector fields in $z$ which are tangential to $M$, then it is clear that

$$
\left|X_{z_{1}} \ldots X_{z_{N}}\{I(w, z)\}\right| \lesssim\left(|w|^{2}+\left|y_{1}\right|\right)^{1 / 2-n} .
$$

These estimates are uniform for $z \in U_{2}$ and $w \in B(0, \eta)$. Therefore, $(K f)(z)$ will be smooth for $z \in U_{2}$ provided that $\left(|w|^{2}+\left|y_{1}\right|\right)^{1 / 2-n}$ is locally integrable in $\mathbf{R}^{2 n-1}$. But this is easy to establish by first integrating out $y_{1}$ and then the rest of the variables.

It is also clear that $K: \mathscr{D}_{M}^{*}\left(U_{1}\right) \rightarrow \delta_{M}^{*}\left(U_{2}\right)$ is a continuous operator. 
This completes the proof that $R$ is a regular kernel.

THE REMAINDER OF THE PROOF OF THEOREM 4.3. Let

$$
\left(K_{\varepsilon} f\right)(z)=\int_{\zeta \in M} \frac{g(\zeta, z) f(\zeta) \chi_{e}(\zeta, z)}{(u \cdot(\zeta-z))^{p}\left({ }^{t} u \cdot(\zeta-z)\right)^{q}(v \cdot(\zeta-z))^{l}}
$$

where we assume $g(\zeta, z)=\mathcal{O}\left(|\zeta-z|^{k}\right)$, for some positive integer $k$. To prove the rest of Theorem 4.3, it suffices to prove the following lemma

4.21. LeMMA. Suppose $v$ is a regular generating function for $V \subset M \times M$. Suppose $U_{1} \times U_{2} \subset V$ and $f \in \mathscr{D}_{M}^{n, n-1}\left(U_{1}\right)$. Then for each $\varepsilon>0, K_{\varepsilon}$ is smooth on $U_{2}$ and the sequence converges as $\varepsilon \rightarrow 0^{+}$in $\mathcal{E}\left(U_{2}\right)$ (i.e. all derivatives converge uniformly on compact subsets of $U_{2}$ ) to a smooth form $K f$, provided the following conditions on $p, q, k, l$, and $n$ are satisfied:

(1) If $n \geqslant 3$ and $p, q \geqslant 1$, then $2 p+2 q+l-k<2 n-1$.

(2) If $q=0$ then $p, l, k$ can be arbitrary nonnegative integers.

(3) If $p=0$ then $q, l, k$ can be arbitrary nonnegative integers.

To see that Lemma 4.21 implies the theorem, we check each kernel:

$E_{123}$. This kernel only makes sense when $n \geqslant 3$ and we note that $p, q>1$ and $p+q=n-1$ and $l=k=1$. The integer $k$ equals one because $u(z, z)={ }^{t} u(z, z)$ and so

$$
u(\zeta, z) \cdot d(\zeta-z) \wedge^{t} u(\zeta, z) \cdot d(\zeta-z)=\theta(|\zeta-z|) .
$$

Therefore, we have $2 p+2 q+l-k=2 n-2<2 n-1$, and so condition (1) is satisfied.

It is easy to see that condition (2) applies to $E_{13}$ and condition (3) applies to $E_{23}$.

To prove Lemma 4.21, we need to show that if $\left(\zeta_{0}, z_{0}\right) \in V$, then there exists an open set $U_{1} \times U_{2} \subset V$ containing $\left(\zeta_{0}, z_{0}\right)$ such that if $f \in \mathscr{D}_{M}^{n, n-1}\left(U_{1}\right)$ then $K_{\varepsilon} f$ is smooth on $U_{2}$ for each $\varepsilon>0$ and that $K_{\varepsilon} f \rightarrow K f$ in $\mathcal{E}\left(U_{2}\right)$ as $\varepsilon \rightarrow 0^{+}$. Then the lemma will follow by a partition of unity argument. We have the following cases to consider on $\left(\zeta_{0}, z_{0}\right)$. Recall that $B=\{(\zeta, z) \in V, v(\zeta, z) \cdot(\zeta-z)=0\}$.

Case $1 .\left(\zeta_{0}, z_{0}\right) \in V-B$ and $p, q, l, k$ can be any positive integers.

Case 2 . $\left(\zeta_{0}, z_{0}\right) \in B-\Delta$ and $p, q, l, k$ can be arbitrary nonnegative integers.

Case 3. $\zeta_{0}=z_{0}$ and $n \geqslant 3$ and $2 p+2 q+l-k<2 n-1$.

Case 4. $\zeta_{0}=z_{0}$ and either $p=0$ or $q=0$. If $q=0$, then $p, l, k$ can be arbitrary nonnegative integers. If $p=0$, then $q, l, k$ can be arbitrary nonnegative integers.

Case 1. Since $B$ is closed, just choose $U_{1} \times U_{2}$ containing $\left(\zeta_{0}, z_{0}\right)$ such that $U_{1} \times U_{2} \cap B=\varnothing$. Then the integrand in $\left(K_{\varepsilon} f\right)(z)$ is smooth.

To handle the remaining cases, we must make the following change of variables:

4.22. Lemma. For each $\left(\zeta_{0}, z_{0}\right) \in V$ with $v \cdot\left(\zeta_{0}-z_{0}\right)=0$, there exist $\eta>0$ and an open set $\tilde{U}_{1} \times \tilde{U}_{2}$ in $\mathbf{C}^{n} \times \mathbf{C}^{n}$ containing $\left(\zeta_{0}, z_{0}\right)$ and a smooth map $\Psi: \tilde{U}_{1} \times \tilde{U}_{2}$ $\rightarrow \mathbf{C}^{n}$ with the following properties:

(a) $\Psi\left(\zeta_{0}, z_{0}\right)=0$.

(b) For each fixed $z \in \tilde{U}_{2}, \Psi_{z}(\zeta)=\Psi(\zeta, z): \Psi_{z}^{-1}\{B(0, \eta)\} \rightarrow B(0, \eta)$ is a diffeomorphism, where $B(0, \eta)$ is the ball in $\mathbf{C}^{n}$ of radius $\eta$. 
(c) If we write $\Psi_{z}(\zeta)=\left(w_{1}(\zeta, z), \ldots, w_{n}(\zeta, z)\right)$, then $\operatorname{Re} w_{1}(\zeta, z)=\rho(\zeta)$ and $w_{2}(\zeta, z)=v(\zeta, z) \cdot(\zeta-z)$.

(d) $\Psi_{z}^{-1}(w)$ is smooth in both $z \in U_{2}$ and $w \in B(0, \eta)$.

(e) If in addition $\zeta_{0}=z_{0}$, then we can also choose $\Psi$ so that $\operatorname{Im} w_{1}(\zeta, z)=$ $\operatorname{Im}\{\hat{u} \cdot(\zeta-z)\}$ and $\Psi_{z}(z)=0$ for each $(z, z) \in \tilde{U}_{1} \times \tilde{U}_{2} \cap M \times M$.

Proof. The proof follows immediately because $v$ is a regular generating function for $V$ (Definition 4.1). See also the proof of Lemma 4.17. Q.E.D.

Case 2. Fix $\left(\zeta_{0}, z_{0}\right) \in B-\Delta$. We must first require that $U_{1} \times U_{2} \cap \Delta=\varnothing$ and that $\left\{U_{1} \times U_{2}\right\} \subset\left\{\tilde{U}_{1} \times \tilde{U}_{2}\right\} \cap M \times M$. Then both $u \cdot(\zeta-z)$ and $\boldsymbol{\prime} u \cdot(\zeta-z)$ are nonvanishing on $U_{1} \times U_{2}$, because $u$ is a strong support function for $M$. We must also require that $\Psi\left\{U_{1} \times U_{2}\right\} \subset B(0, \eta)$. If $f \in \mathscr{D}_{M}^{n, n-1}\left(U_{1}\right)$, then the $w$-support of $f\left(\Psi_{z}^{-1}(w)\right)$ will be contained in $B(0, \eta)$ for each $z \in U_{2}$. Pulling back the integral in $\left(K_{\mathrm{s}} f\right)(z)$, we obtain

$$
\left(K_{\varepsilon} f\right)(z)=\int_{\left|w_{2}\right|>\varepsilon} \frac{G(w, z)}{w_{2}^{l}} d \lambda(w)
$$

where $d \lambda(w)$ is Lebesgue measure on $\mathbf{R}^{2 n-1}$ and

$$
G(w, z)=\Psi_{z}^{-1 *}\left\{\frac{g f}{(u \cdot(\zeta-z))^{p}\left({ }^{t} u \cdot(\zeta-z)\right)^{q}}\right\}(w) .
$$

Clearly, $G$ is smooth and for each $z \in U_{2}$, the $w$-support of $G(w, z)$ is contained in $B(0, \eta)$.

Since the domain of integration in (4.23) no longer depends on $z$, we can differentiate in $z$ under the integral sign to show $\left(K_{8} f\right)$ is smooth on $U_{2}$, for each $\varepsilon>0$.

We now have a one complex variable lemma found in Harvey-Polking [6, Lemma (5.19)].

4.24. LeMMA. For each positive integer $l$, there is a constant $M_{l}$ depending only on $l$ such that

$$
\left|\int_{\substack{w_{2} \in \mathbf{C} \\\left|w_{2}\right|>\varepsilon}} w_{2}^{-l} \Phi\left(w_{2}\right) d \lambda\left(w_{2}\right)-\int_{\substack{w_{2} \in \mathbf{C} \\\left|w_{2}\right|>\varepsilon}} \frac{1}{(l-1) !} w_{2}^{-1}\left(\frac{\partial}{\partial w_{2}}\right)^{l-1}\left\{\Phi\left(w_{2}\right)\right\} d \lambda\left(w_{2}\right)\right|
$$

for each $\Phi \in \mathscr{D}(\mathrm{C})$ and where

$$
\|\Phi\|_{l-1}=\int_{\substack{|\alpha|<l-1 \\ w_{2} \in C}}\left|D_{w_{2}}^{\alpha} \Phi\left(w_{2}\right)\right|
$$

and where $d \lambda\left(w_{2}\right)$ is Lebesgue measure on $\mathbf{C} .\left(D_{w_{2}}^{\alpha}\right.$ is a differential operator in $w_{2}$ of order $|\alpha|$.) 
Remark. If we denote the principal value limit of $w_{2}^{-l}$ by P.V. $\left(w_{2}^{-l}\right)$, then Lemma 4.24 says that

$$
\frac{(-1)^{l-1}}{(l-1) !}\left(\frac{\partial}{\partial w_{2}}\right)^{l-1}\left\{\text { P.V. }\left(w_{2}^{-1}\right)\right\}=\text { P.V. }\left(w_{2}^{-l}\right) \text {. }
$$

We now use the following notation. For $w \in \mathbf{C}^{n}$, we let $w^{\prime \prime}=\left(w_{3}, \ldots, w_{n}\right) \in$ $\mathbf{C}^{n-2}$ and $t=\left(y_{1}, w^{\prime \prime}\right) \in \mathbf{R}^{2 n-3}$ where $y_{1}=\operatorname{Im} w_{1}$. If we identify $\mathbf{R}^{2 n-1}$ with the set $\left\{w \in C^{n}, \operatorname{Re} w_{1}=0\right\}$, then $\mathbf{R}^{2 n-1}$ has coordinates $\left(w_{2}, t\right)$. To prove case (2), we apply Lemma 4.24 to $\Phi\left(w_{2}, z\right)=\int G\left(w_{2}, t, z\right) d \lambda(t)$ where $d \lambda(t)$ is Lebesgue measure on $\mathbf{R}^{2 n-3}$. Clearly, $\Phi$ is smooth and for each $z \in U_{2}$, the $w_{2}$-support of $\Phi\left(w_{2}, z\right)$ will be contained in the set $\left\{w_{2} \in \mathbf{C} ;\left|w_{2}\right|<\eta\right\}$. It is also clear that $\left\|\Phi\left(w_{2}, z\right)\right\|_{l-1} \leqslant C$, where $C$ is a constant independent of $z \in U_{2}$. We have

$$
\left(K_{\mathrm{s}} f\right)(z)=\int_{\left|w_{2}\right|>\varepsilon} w_{2}^{-l} \Phi\left(w_{2}, z\right) d \lambda\left(w_{2}\right)
$$

and Lemma 4.24 implies $\left(K_{e} f\right)(z) \rightarrow(K f)(z)$ uniformly for $z \in U_{2}$, where

$$
(K f)(z)=\frac{1}{(l-1) !} \int w_{2}^{-1}\left(\frac{\partial}{\partial w_{2}}\right)^{l-1}\left\{\Phi\left(w_{2}, z\right)\right\} d \lambda\left(w_{2}\right) .
$$

Note that $(K f)(z)$ is smooth for $z \in U_{2}$ because $\Phi$ is smooth and $w_{2}^{-1}$ is locally integrable on $\mathbf{C}$.

If $D_{z}^{\beta}$ is a differential operator in $z$ of order $|\beta|$, then clearly $\left\|\left(D_{z}^{\beta} \Phi\right)\left(w_{2}, z\right)\right\|_{l-1}$ $<C_{\beta}$, where $C_{\beta}$ is a constant independent of $z \in U_{2}$. Applying Lemma 4.24 to $D_{z}^{\beta} \Phi\left(w_{2}, z\right)$, we conclude that $D_{z}^{\beta}\left(K_{\varepsilon} f\right)(z) \rightarrow D_{z}^{\beta}(K f)(z)$ uniformly for $z \in U_{2}$. This completes the proof of Case 2.

Case 3. Fix $z_{0} \in M$ with $\left(z_{0}, z_{0}\right) \in V$. In Cases 3 and 4, we will be working near the diagonal. Therefore, since $u=h \hat{u}$, we may replace $u$ by $\hat{u}$ in the definition of $K f$.

We shall use the change of variables $w=\Psi_{z}(\zeta)$ described in Lemma 4.22, where we assume that $\Psi_{z}(\zeta)$ also satisfies part (e). If we identify $\mathbf{R}^{2 n-1}$ with $\left\{w \in \mathbf{C}^{n}\right.$; $\left.\operatorname{Re} w_{1}=0\right\}$ then $\Psi_{z}^{-1}\left\{\mathbf{R}^{2 n-1}\right\} \subset M$. We also have $\Psi_{z}^{-1}(0)-z=0$ for each $z \in M$ $\cap \tilde{U}_{2}$, and a Taylor series expansion about $w=0$ yields

$$
\left|\Psi_{z}^{-1}(w)-z\right| \approx|w| \quad \text { for }|w| \leqslant \eta, \operatorname{Re} w_{1}=0 \text { and } z \in M \cap \tilde{U}_{2} .
$$

Pulling back the integral in $K_{\varepsilon} f$ via $\Psi_{z}^{-1}$, we obtain

$$
\left(K_{8} f\right)(z)=\int_{\left|w_{2}\right|>\varepsilon} \frac{g_{1}(w, z) f_{1}(w, z) d \lambda(w)}{u_{1}(w, z)^{p}\left({ }^{l} u_{1}(w, z)\right)^{q} w_{2}^{l}}
$$

where $d \lambda(w)$ is Lebesgue measure on $\mathbf{R}^{2 n-1}$ and

$$
\begin{aligned}
g_{1}(w, z) d \lambda(w) & =\Psi_{z}^{-1 *}\{g\}(w), \\
u_{1}(w, z) & =u\left(\Psi_{z}^{-1}(w), z\right) \cdot\left(\Psi_{z}^{-1}(w)-z\right)=\operatorname{Re} u_{1}+i y_{1}, \\
{ }^{t} u_{1}(w, z) & ={ }^{t} u\left(\Psi_{z}^{-1}(w), z\right) \cdot\left(\Psi_{z}^{-1}(w)-z\right), \\
f_{1}(w, z) & =f\left(\Psi_{z}^{-1}(w)\right) .
\end{aligned}
$$


For each $\varepsilon>0$, it is clear that $\left(K_{\varepsilon} f\right)$ is smooth on $M \cap \tilde{U}_{2}$. As in the proof of the regularity for the $R$ kernel, we need the following lemma:

4.26. LemMA. For each $z_{0} \in M$ with $\left(z_{0}, z_{0}\right) \in V$, there exist $\eta>0$ and an open set $U_{2} \subset M$ containing $z_{0}$, such that the following estimates hold uniformly for $w \in \mathbf{R}^{2 n-1}$ with $|w| \leqslant \eta$ and for $z \in U_{2}$.

(a) $g_{1}(w, z)=\theta\left(|w|^{k}\right)$.

(b) $u_{1}(w, z) \approx|w|^{2}+\left|y_{1}\right|,{ }^{t} u_{1}(w, z) \approx|w|^{2}+\left|y_{1}\right|, u_{1}-{ }^{t} u_{1}=\theta\left(|w|^{2}\right)$.

(c) If $X_{i_{z}}$ are vector fields tangential to $M$, for $1<i<N$, then

$$
\begin{aligned}
& X_{1_{z}} \ldots X_{N_{z}}\left\{u_{1}(w, z)\right\}=\theta\left(|w|^{2}\right), \\
& X_{1_{z}} \ldots X_{N_{z}}\left\{{ }^{t} u_{1}(w, z)\right\}=\theta\left(|w|^{2}\right), \\
& X_{1_{z}} \ldots X_{N_{z}}\left\{g_{1}(w, z)\right\}=\theta\left(|w|^{k}\right) .
\end{aligned}
$$

(d) If $D_{w_{2}}^{\alpha}$ is a differential operator in $w_{2}$ of order $|\alpha|$, then

$$
\begin{aligned}
D_{w_{2}}^{\alpha} u_{1}(w, z) & = \begin{cases}\Theta\left(|w|^{2-|\alpha|}\right) & \text { if }|\alpha|<2, \\
\Theta(1) & \text { if }|\alpha|>2 ;\end{cases} \\
D_{w_{2}}^{\alpha}{ }^{t} u_{1}(w, z) & = \begin{cases}\Theta\left(|w|^{2-|\alpha|}\right) & \text { if }|\alpha|<2, \\
\Theta(1) & \text { if }|\alpha|>2 ;\end{cases} \\
D_{w_{2}}^{\alpha} g_{1}(w, z) & = \begin{cases}\Theta\left(|w|^{k-|\alpha|}\right) & \text { if }|\alpha|<k, \\
\Theta(1) & \text { if }|\alpha|>k .\end{cases}
\end{aligned}
$$

Proof. The proof of parts (a) through (c) is identical to the proofs in Lemma 4.19. The proof of part (d) is clear from part (a) and by noting that if $l$ is any positive integer, then

$$
D_{w_{2}}^{\alpha} \vartheta\left(|w|^{l}\right)= \begin{cases}\vartheta\left(|w|^{l-|\alpha|}\right) & \text { if }|\alpha|<l \\ \vartheta(1) & \text { if } \mid \alpha>l .\end{cases}
$$

(See notation at the beginning of Chapter 3.) Q.E.D.

Choose $\eta>0$ to satisfy Lemmas 4.26 and 4.22 and choose $U_{2} \subset M$ to satisfy Lemma 4.26. By shrinking $U_{2}$ if necessary, there exists an open set $U_{1} \subset M$ containing $z_{0}$ such that $\Psi\left\{U_{1} \times U_{2}\right\} \subset B(0, \eta)$.

If we require $f(\zeta)$ to have compact support in $U_{1}$, then for each $z \in U_{2}$, the $w$-support of $f_{1}(w, z)$ will be contained in $B(0, \eta)$.

Let

$$
I(w, z)=\frac{g_{1}(w, z) f_{1}(w, z)}{u_{1}(w, z)^{p}\left({ }^{t} u_{1}(w, z)\right)^{q}} .
$$

Since $u_{1}(w, z) \neq 0$ and ${ }^{t} u_{1}(w, z) \neq 0$ for $w \neq 0, I(w, z)$ is smooth for $w \neq 0$. Let

$$
\Phi\left(w_{2}, z\right)=\int_{t \in \mathbf{R}^{2 n-3}} I\left(w_{2}, t, z\right) d \lambda(t)
$$

where we have used the same notation as in Case 2. It is clear that if $z \in U_{2}$, then the w-support of $I(w, z)$ and hence the $w$-support of $\Phi\left(w_{2}, z\right)$ is contained in $B(0, \eta)$. 
We have $\left(K_{\varepsilon} f\right)(z)=\int_{\left|w_{2}\right| \geqslant e} \Phi\left(w_{2}, z\right) w_{2}^{-l} d \lambda\left(w_{2}\right)$ and we define

$$
(K f)(z)=\frac{1}{(l-1) !} \int\left(\frac{\partial}{\partial w_{2}}\right)^{l-1}\left\{\Phi\left(w_{2}, z\right)\right\} w_{2}^{-1} d \lambda\left(w_{2}\right) .
$$

We wish to show that $(K f)$ is smooth on $U_{2}$ and that $K_{\varepsilon} f \rightarrow K f$ in $\mathcal{E}\left(U_{2}\right)$ as $\varepsilon \rightarrow 0^{+}$. Suppose $X_{i_{2}}$ are vector fields tangential to $M$ for $1 \leqslant i \leqslant N$. We shall estimate $\left\|X_{1_{z}} \ldots X_{N_{z}} \Phi\left(w_{2}, z\right)\right\|_{l-1}$ uniformly for $z \in U_{2}$ and then apply Lemma 4.24.

First, suppose that $D_{w_{2}}$ is a first order differential operator in $w_{2}$. Lemma 4.26 implies

$$
\left|D_{w_{2}} g_{1}(w, z)\right|=\mathcal{O}\left(|w|^{k-1}\right) \lesssim\left(|w|^{2}+\left|y_{1}\right|\right)^{k / 2-1 / 2}
$$

and

$$
\begin{aligned}
\left|D_{w_{2}}\left\{u_{1}^{-p}\right\}\right| & =\left|p\left(D_{w_{2}} u_{1}\right) u_{1}^{-(p+1)}\right| \\
& =\Theta(|w|)\left(|w|^{2}+\left|y_{1}\right|\right)^{-(p+1)} \\
& \lesssim\left(|w|^{2}+\left|y_{1}\right|\right)^{-(p+1 / 2)} .
\end{aligned}
$$

Similarly, we have $\left|D_{w_{2}}\left\{\left({ }^{t} u_{1}\right)^{-q}\right\}\right| \lesssim\left(|w|^{2}+\left|y_{1}\right|\right)^{-(q+1 / 2)}$. Therefore, we have the following estimate on $D_{w_{2}}\{I\}$ :

$$
\left|D_{w_{2}}\left\{I\left(w_{2}, t, z\right)\right\}\right| \lesssim\left(\left|w^{\prime \prime}\right|^{2}+\left|y_{1}\right|\right)^{-[p+q+1 / 2-k / 2]}
$$

Repeated use of Lemma 4.24 shows that if $D_{w_{2}}^{\beta}$ is a differential operator in $w_{2}$ of order $|\beta| \leqslant l-1$, then $\left|D_{w_{2}}^{\beta}\left\{I\left(w_{2}, t, z\right)\right\}\right| \leqslant\left(\left|w^{\prime \prime}\right|^{2}+\left|y_{1}\right|\right)^{-\mu}$ where $\mu=p+q+$ $(l-1) / 2-k / 2$. Moreover, if we apply the vector field $X_{i_{z}}$ to $I$, Lemma 4.26 implies the estimates not to worsen, i.e.

$$
\left|D_{w_{2}}^{\beta} X_{1_{z}} \ldots X_{N_{z}}\left\{I\left(w_{2}, t, z\right)\right\}\right| \lesssim\left(\left|w^{\prime \prime}\right|^{2}+\left|y_{1}\right|\right)^{-\mu}
$$

These estimates are uniform for $z \in U_{2}$ and for $|w|=\left(\left|w_{2}\right|^{2}+|t|^{2}\right)^{1 / 2}<\eta$.

In view of (4.27) and (4.29) we must determine which values of $\mu$ make the function $\left(\left|w^{\prime \prime}\right|^{2}+\left|y_{1}\right|\right)^{-\mu}$ locally integrable on $\mathbf{R}^{2 n-3}$, in order to get estimates on $\left\|X_{1_{z}} \ldots X_{N_{z}}\left\{\Phi\left(w_{2}, z\right)\right\}\right\|_{l-1}$. By integrating out $y_{1}$ first and then $w^{\prime \prime}$, it is easy to show that $\left(\left|w^{\prime \prime}\right|^{2}+\left|y_{1}\right|\right)^{-\mu}$ is locally integrable on $\mathbf{R}^{2 n-3}$ if $2 \mu<2 n-2$. Since $\mu=p+q+(l-1) / 2-k / 2$, this inequality will be satisfied if $2 p+2 q+l-k$ $<2 n-1$. But this is precisely the condition assumed in Case 3.

To summarize, the condition on $p, q, l, k, n$ assumed in Case 3 implies that there is a constant $C^{\prime}$, which is independent of $z \in U_{2}$ such that

$$
\left\|X_{1_{z}} \ldots X_{N_{z}}\left\{\Phi\left(w_{2}, z\right)\right\}\right\|_{l-1} \leqslant C^{\prime}
$$

Since $w_{2}^{-1}$ is locally integrable on $\mathbf{C}$ and $\Phi$ has compact $w_{2}$-support, the estimate in (4.30) implies that $K f$ is smooth on $U_{2}$ (see (4.28)). Now Lemma 4.24 implies that $X_{1} \ldots X_{N}\left\{K_{8} f\right\} \rightarrow X_{1} \ldots X_{N}\{K f\}$ uniformly on $U_{2}$. This completes the proof of Case 3.

Case 4. Let us assume $q=0$. The case when $p=0$ is similar. In this case

$$
\left(K_{\varepsilon} f\right)(z)=\int_{\left|w_{2}\right|>\varepsilon} \frac{g_{1}(w, z) f_{1}(w, z)}{u_{1}(w, z)^{p} w_{2}^{l}} d \lambda(w)
$$


where we have used the same notation as in Case 3. Now $u_{1}(w, z)=\operatorname{Re} u_{1}(w, z)+$ iy 1 . Let

$$
h(w, z)=\left[\frac{\partial}{\partial y_{1}} u_{1}(w, z)\right]^{-1}=\left[\frac{\partial}{\partial y_{1}} \operatorname{Re} u_{1}+i\right]^{-1} .
$$

It is clear that $h(w, z)$ is smooth because $\partial \operatorname{Re} u_{1} / \partial y_{1}+i \neq 0$. Note that

$$
\frac{-h}{p-1} \frac{\partial}{\partial y_{1}}\left\{u_{1}^{1-p}\right\}=u_{1}^{-p}
$$

So we may integrate by parts with $\partial / \partial y_{1}$ to obtain

$$
\left(K_{8} f\right)(z)=\int_{\left|w_{2}\right|>\varepsilon} \frac{G_{1}(w, z)}{u_{1}^{p-1} w_{2}^{-l}} d \lambda(w)
$$

where $G_{1}$ is smooth and for each $z \in U_{2}$, the $w$-support of $G_{1}(w, z)$ is contained in $B(0, \eta)$. Iterating this procedure $p$-times, we obtain

$$
\left(K_{8} f\right)(z)=\int_{\left|w_{2}\right|>\varepsilon} \log \left\{u_{1}(w, z)\right\} G_{2}(w, z) w_{2}^{-l} d \lambda(w)
$$

where $G_{2}$ is smooth with $w$-support contained in $B(0, \eta)$. Strict pseudoconvexity of $M$ implies that $\operatorname{Re}\{\hat{u}(\zeta, z) \cdot(\zeta-z)\}>0$ for $(\zeta, z) \in M \times M$ with $0<|\zeta-z|<$ $\eta$. Therefore, $\operatorname{Re}\left\{u_{1}(w, z)\right\}>0$ for $0<|w|<\eta$ and $z \in U_{2}$. Hence, a branch of $\log \left\{u_{1}(w, z)\right\}$ exists for $0<|w|<\eta$ and $z \in U_{2}$.

We can even continue further. Note that

$$
h \frac{\partial}{\partial y_{1}}\left\{u_{1} \log u_{1}-u_{1}\right\}=\log u_{1} \text {. }
$$

So, we may integrate by parts again with $\partial / \partial y_{1}$ and obtain

$$
\left(K_{8} f\right)(z)=\int_{\left|w_{2}\right|>\varepsilon}\left[u_{1}(w, z) \log u_{1}(w, z)-u_{1}(w, z)\right] G_{3} w_{2}^{-1} d \lambda(w)
$$

where $G_{3}$ is smooth. Let $Q$ be a large positive integer to be determined later. If we iterate this process $Q$ times, we obtain

$$
\left(K_{\varepsilon} f\right)(z)=\int_{\left|w_{2}\right|>\varepsilon}\left[U_{Q}(w, z) \log u_{1}(w, z)-C(w, z)\right] G_{4} w_{2}^{-l} d \lambda(w),
$$

where $C$ and $G_{4}$ are smooth and the $w$-support of $G_{4}(w, z)$ is contained in $B(0, \eta)$. $U_{Q}$ is some smooth function satisfying $U_{Q}=\theta\left(\left|u_{1}\right|^{Q}\right)$.

We let $I(w, z)=\left[U_{Q} \log u_{1}-C\right] G_{4}$ and

$$
\Phi\left(w_{2}, z\right)=\int_{t \in \mathbf{R}^{2 n-3}} I\left(w_{2}, t, z\right) d \lambda(t)
$$

where we have used the notation in Cases 2 and 3. We have $\left(K_{8} f\right)(z)=$ $\int_{\left|w_{2}\right|>e} \Phi\left(w_{2}, z\right) w_{2}^{-l} d \lambda\left(w_{2}\right)$ and we define

$$
(K f)(z)=\frac{1}{(l-1) !} \int\left(\frac{\partial}{\partial w_{2}}\right)^{l-1}\left\{\Phi\left(w_{2}, z\right)\right\} w_{2}^{-1} d \lambda\left(w_{2}\right) .
$$

As in Case 3, we need to estimate $\left\|X_{1_{z}} \ldots X_{N_{z}}\left\{\Phi\left(w_{2}, z\right)\right\}\right\|_{l-1}$ uniformly for $z \in U_{2}$. 
Let $D_{w_{2}}^{\beta}$ be a differential operator in $w_{2}$ of order $|\beta|<l-1$. In view of (4.31), we must estimate $\left|D_{w_{2}}^{\beta} X_{1_{z}} \ldots X_{N_{z}}\left\{I\left(w_{2}, t, z\right)\right\}\right|$. But

$$
D_{w_{2}}^{\beta} X_{1_{z}} \ldots X_{N_{z}}\left\{U_{Q}(w, z) \log u_{1}(w, z)\right\}=\vartheta\left(\left|u_{1}\right|^{Q-N-l+1}\right) .
$$

So, once $N$ and $l$ are fixed, then $Q$ can be chosen so that $Q-N-l>0$. With this choice of $Q$, we have $\left|D_{w_{2}}^{\beta} X_{1_{z}} \ldots X_{N_{z}}\left\{I\left(w_{2}, t, z\right)\right\}\right| \lesssim 1$. This estimate holds uniformly for $z \in U_{2}$. Therefore, there is a constant $C_{1}$ independent of $z \in U_{2}$, such that

$$
\left\|X_{1_{z}} \ldots X_{N_{z}}\left\{\Phi\left(w_{2}, z\right)\right\}\right\|_{l-1} \leqslant C_{1} .
$$

For each $z \in U_{2}$, the $w_{2}$-support of $\Phi\left(w_{2}, z\right)$ is contained in $B(0, \eta)$. Thus, from (4.32), $K f$ is smooth on $U_{2}$ and Lemma 4.24 implies that $K_{8} f \rightarrow K f$ in $\mathcal{E}\left(U_{2}\right)$.

This completes the proof of Case 4 and of Theorem 4.3. Q.E.D.

Remarks. 1. We note that to prove Theorem 4.3 for the kernels used in $\$ 4.2$ to solve the $\bar{\partial}_{M}$ equation, we need only use Lemma 4.21 with $l=1$. To prove Lemma 4.21 when $l=1$, it is unnecessary to use Lemma 4.24 because $w_{2}^{-1}$ is locally integrable in the complex plane.

2. Cases 1, 2, and 4 do not use the full strength of part (c) in the definition of a strong support function $u$. All that is used is that $u(\zeta, z) \cdot(\zeta-z) \neq 0$ and ${ }^{t} u(\zeta, z) \cdot(\zeta-z) \neq 0$ for $\zeta \neq 0$, and in Case 4, we used that $\operatorname{Re}\{\hat{u}(\zeta, z) \cdot(\zeta-z)\}$ $>0$ for $\zeta \neq z$. These three cases did not use the following estimate:

$$
|\operatorname{Re} u \cdot(\zeta-z)| \gtrsim|\zeta-z|^{2} \text { for }|\zeta-z| \text { small. }
$$

Since the principal value limits of the kernels $E_{13}$ and $E_{23}$ only depend on Cases 1, 2, and 4 of Lemma 4.21, we need not assume that (4.33) holds to prove that P.V.E $E_{23}$ and P.V. $E_{13}$ are regular kernels.

However, the regularity proofs for the kernels $R$ and $E_{123}$ definitely use (4.33), because both $u \cdot(\zeta-z)$ and ${ }^{t} u \cdot(\zeta-z)$ appear on the denominators of these kernels.

\section{Chapter 5. The SOlution to $\bar{\partial}_{S}$ IN CODIMENSION TWO}

Suppose $M=\partial D$ is the boundary of a strictly pseudoconvex domain and suppose $S \subset M$ is an oriented hypersurface in $M$ defined by $S=\{z \in M$; $\operatorname{Re} h(z)=0\}$ where $h$ is holomorphic near $\bar{D}$. In this chapter, we construct an operator $E$ : $\mathscr{Q}_{S}^{p, q}\left(S-C_{S}\right) \rightarrow \mathcal{E}_{S}^{p, q-1}\left(S-C_{S}\right)$ which is also a continuous map $E$ : $\mathcal{L}_{p, \text { comp }}^{*}\left(S-C_{S}\right) \rightarrow \mathcal{L}_{p, \text { loc }}^{*}\left(S-C_{S}\right), 1 \leqslant p \leqslant \infty$, and such that $\bar{\partial}_{s}\{E(f)\}+E\left(\bar{\partial}_{s} f\right)$ $=f$ for $f \in \mathscr{Q}_{S}^{p, q}\left(S-C_{S}\right)$. The operator $E$ will be a boundary value jump across $S$ of the kernel $E_{123}$ used in Chapter 4 . The hypersurface $S$ is the boundary of the type of region $\omega$ considered in Chapter 4. However, we need not assume $S$ is compact, nor must we assume that $S$ has admissible boundary.

5.1. Boundary value theorems. Let $E$ denote any one of the kernels $E_{123}, E_{13}, E_{23}$, $R, L$ or ${ }^{t} L$. If $z \in M$ is fixed, then recall that the $\zeta$-singular support of $E(\zeta, z)$ is contained in the set $B_{z}=\{\zeta \in M ; h(\zeta)=h(z)\}$. Let $r=\operatorname{Re} h$ and $S_{t}=\{z \in M$; $r(z)=t\}$. If $r(z)=t$, then it is clear that $B_{z} \subset S_{t}$. Therefore, if $f \in \mathscr{Q}_{S}^{p, q}\left(S-C_{S}\right)$, 
then

$$
E\left([S]^{0,1} \wedge f\right)(z)=\int_{\zeta \in S} E(\zeta, z) \wedge f(\zeta)
$$

defines a smooth form for $z \in M-S$. Our boundary value theorems (Theorems 5.2 and 5.3) examine the smoothness of $E\left([S]^{0,1} \wedge f\right)(z)$ as $z$ approaches $S$.

In general, suppose $r$ is an arbitrary smooth function on $M$. We let $M^{+}=\{z \in$ $M ; r(z)>0\}$ and $M^{-}=\{z \in M ; r(z)<0\}$ and $S=\{z \in M ; r(z)=0\}$. We need the following local definition.

5.1. Definition. Suppose $F$ is a smooth form on $M-S$ and let $z_{0} \in S$. Let $s$ be a nonnegative integer and let $X_{1}, \ldots, X_{N}$ be arbitrary vector fields which are tangential to $S$. We say that $F \in C^{s}\left(\overline{M_{z_{0}}^{+}}\right)$(resp. $F \in C^{s}\left(\overline{M_{z_{0}}^{-}}\right)$) if there exists an open set $U$ in $M$ containing $z_{0}$, such that $X_{1} \ldots X_{N}\{F\}$ is $s$-times continuously differentiable on $\overline{M^{+} \cap U}$ (resp. on $\overline{M^{-} \cap U}$ ).

A vector field $X$ is tangential to $S$ if $X\{r\}=0$.

If $F \in C^{s}\left(\overline{M_{z_{0}}^{+}}\right)$then near $z_{0}$ we can take continuous boundary values to $S$ from $\mathrm{M}^{+}$of any number of derivatives of $F$ which are tangential to $S$, but we can only take boundary values of $s$ normal derivatives of $F$ (normal to $S$ but tangential to $M)$.

Notation. Suppose $F \in C^{s}\left(\overline{M_{z_{0}}^{ \pm}}\right)$for each $z_{0} \in S-C_{S}$. We denote by $F^{ \pm}$the continuous extension to $S-C_{S}$ of $\left.(F)_{T_{S}}\right|_{M^{ \pm}}$. Note that $F^{ \pm} \in \mathcal{E}_{S}^{p, q}\left(S-C_{S}\right)$.

The next two theorems are our principal results concerning the boundary values of our kernels.

5.2. TheOREM. Suppose $S$ is an arbitrary oriented smooth hypersurface in $M$ and suppose $z_{0} \in S-C_{S}$. Suppose $f \in \mathcal{E}_{S}^{p, q}$; then

(a) $R\left([S]^{0,1} \wedge f\right)_{T_{S}} \in C^{0}\left(\overline{M_{z_{0}}^{+}}\right) \cap C^{0}\left(\overline{M_{z_{0}}^{-}}\right)$.

(b) If $f \in \mathcal{E}_{S}^{p, 0}(S)$ and $\bar{\partial}_{S} f \equiv 0$ near $z_{0}$, then

$$
R\left([S]^{0,1} \wedge f\right) \in C^{\infty}\left(\overline{M_{z_{0}}^{+}}\right) \cap C^{\infty}\left(\overline{M_{z_{0}}}\right) .
$$

(c) ${ }^{t} L\left([S]^{0,1} \wedge f\right) \in C^{\infty}\left(\overline{M_{z_{0}}^{+}}\right) \cap C^{\infty}\left(\overline{M_{z_{0}}^{-}}\right)$.

5.3. ThEOREM. Suppose $S \subset M=\partial D$ is an oriented hypersurface defined by

$$
S=\{z \in M ; \operatorname{Re} h(z)=0\}
$$

where $h$ is holomorphic near $\bar{D}$. Suppose $f \in \mathscr{D}_{S}^{p, q}\left(S-C_{S}\right)$ and $z_{0} \in S-C_{S}$. Then

(a) $E_{13}\left([S]^{0,1} \wedge f\right), E_{23}\left([S]^{0,1} \wedge f\right) \in C^{\infty}\left(\overline{M_{z_{0}}^{+}}\right) \cap C^{\infty}\left(\overline{M_{z_{0}}^{-}}\right)$.

(b) $E_{123}\left([S]^{0,1} \wedge f\right) \in C^{0}\left(\overline{M_{z_{0}}^{+}}\right) \cap C^{0}\left(\overline{M_{z_{0}}^{-}}\right)$.

Remarks. 1. Note that $S$ can be any smooth oriented hypersurface in $M$ for Theorem 5.2. This is because the singular set in $M \times M$ for the $R$ kernel is the diagonal. The additional hypothesis on $S$ in Theorem 5.3 is essential.

2. In Theorem 5.2, we know nothing about the boundary behavior of $R\left([S]^{0,1} \wedge f\right)_{N_{s}}$. However, in [2], the author has proved that if $M$ is the unit sphere in $\mathrm{C}^{n}$, then $R\left([S]^{0,1} \wedge f\right) \in C^{\infty}\left(\overline{M_{z_{0}}^{+}}\right) \cap C^{\infty}\left(\overline{M_{z_{0}}^{-}}\right)$. The proof uses entirely different techniques and exploits the fact that the $R$ kernel on the sphere satisfies $\bar{\partial}_{M}^{*} \circ R=0$. 
We shall postpone the proofs of Theorems 5.2 and 5.3 until $\$ 5.4$.

Let $E$ be any one of our kernels discussed above. Theorems 5.2 and 5.3 imply that if $f \in \mathscr{Q}_{S}^{p, q}\left(S-C_{S}\right)$, then $\left.E\left([S]^{0,1} \wedge f\right)_{T_{S}}\right|_{M^{ \pm}}$has a smooth extension to $S-C_{S}$. We denote their extension by $E^{ \pm}(f) \in \mathcal{E}_{S}^{*}\left(S-C_{S}\right)$.

5.2. Plemelj jump formula for the $R$ kernel. In this section, we prove our global and local theorems for the jump formula for the $R$ kernel. This formula is analogous to the classical Plemelj jump formulas for the Cauchy kernel on the complex plane and for the Bochner-Martinelli kernel on $\mathbf{C}^{n}$. In this section $S$ will be an arbitrary smooth oriented hypersurface in $M$ defined by $S=\{z \in M$; $r(z)=0\}$.

5.4. TheOrem. Suppose $f \in \mathscr{D}_{S}^{p, q}\left(S-C_{S}\right)$ with $0 \leqslant q \leqslant n-3$, or if $q=n-2$, then assume $f$ satisfies condition $\theta$ for $S$. Then

(a) $R^{+}(f)-R^{-}(f)=f$.

(b) If in addition $\bar{\partial}_{S} f \equiv 0$, then $\bar{\partial}_{M}\left\{R\left([S]^{0,1} \wedge f\right)\right\} \equiv 0$ on $M-S$.

Proof. (a) First, we develop some preliminaries. We want to construct a sequence of smooth forms on $M$ which approximate the 'boundary jump' across $S$. To do this let $\psi_{t}: \mathbf{R} \rightarrow[0,1]$ be a sequence of smooth functions such that $\psi_{t}(x)=1$ for $|x| \geqslant 2 t$, and $\psi_{t}(x)=0$ for $|x| \leqslant t$. We let $\eta_{t}: M \rightarrow[0,1]$ be defined by $\eta_{t}(z)=\psi_{t}(r(z))$. It is clear that $\eta_{t} \rightarrow 1$ (weakly) as $t \rightarrow 0$ and that $\eta_{t} \equiv 0$ near $S$, for each $t>0$. If $F \in C^{0}\left(\overline{M_{z_{0}}^{+}}\right) \cap C^{0}\left(\overline{M_{z_{0}}^{-}}\right)$for each $z_{0} \in S-C_{S}$, then it is clear that

$$
\bar{\partial}_{M} \eta_{t} \wedge F \rightarrow[S]^{0,1} \wedge\left(F^{+}-F^{-}\right)
$$

weakly as $t \rightarrow 0$.

Since $R\left([S]^{0,1} \wedge f\right)$ is a current with locally integrable coefficients on $M$, we have $\eta_{t} R\left([S]^{0,1} \wedge f\right) \rightarrow R\left([S]^{0,1} \wedge f\right)$ weakly on $M$, as $t \rightarrow 0$. Taking $\bar{\partial}_{M}$ of both sides, and using Theorem 3.14, we conclude that

$$
\bar{\partial}_{M} \eta_{t} \wedge R\left([S]^{0,1} \wedge f\right)+\eta_{t} R\left([S]^{0,1} \wedge \bar{\partial}_{S} f\right) \rightarrow[S]^{0,1} \wedge f+R\left([S]^{0,1} \wedge \bar{\partial}_{S} f\right)
$$

as $t \rightarrow 0$. However, since $R\left([S]^{0,1} \wedge \bar{\partial}_{S} f\right)$ has integrable coefficients on $M$,

$$
\eta_{t} R\left([S]^{0,1} \wedge \bar{\partial}_{S} f\right) \rightarrow R\left([S]^{0,1} \wedge \bar{\partial}_{S} f\right)
$$

as $t \rightarrow 0$. Therefore, we have $\bar{\partial}_{M} \eta_{t} \wedge R\left([S]^{0,1} \wedge f\right) \rightarrow[S]^{0,1} \wedge f$. The orthogonal decomposition in (2.16) yields

$$
R\left([S]^{0,1} \wedge f\right)=R\left([S]^{0,1} \wedge f\right)_{T_{S}}+\phi \wedge R\left([S]^{0,1} \wedge f\right)_{N_{S}}
$$

near $S-C_{\underline{S}}$. However, $\bar{\partial}_{M} \eta_{t}=\left(\psi_{\underline{t}}^{\prime} \circ r\right)\left(\bar{\partial}_{M} r\right)$ and (2.13) implies $\phi=\phi_{t_{M}}=\delta \bar{\partial}_{M} r$. Therefore, $\bar{\partial}_{M} \eta_{t} \wedge \phi=0$ and so $\bar{\partial}_{M} \eta_{t} \wedge R\left([S]^{0,1} \wedge f\right)=\bar{\partial}_{M} \eta_{t} \wedge R\left([S]^{0,1} \wedge f\right)_{T_{S}}$. Thus,

$$
\bar{\partial}_{M} \eta_{t} \wedge R\left([S]^{0,1} \wedge f\right)_{T_{S}} \rightarrow[S]^{0,1} \wedge f \text { as } t \rightarrow 0
$$

Theorem 5.2 implies that $R\left([S]^{0,1} \wedge f\right)_{T_{S}} \in C^{0}\left(\overline{M_{z_{0}}^{+}}\right) \cap C^{0}\left(\overline{M_{z_{0}}^{-}}\right)$. Thus $\bar{\partial}_{M} \eta_{t} \wedge$ $R\left([S]^{0,1} \wedge f\right)_{T_{S}} \rightarrow[S]^{0,1} \wedge\left(R^{+} f-R^{-} f\right)$ as $t \rightarrow 0$, and we conclude that $R^{+} f-$ $R^{-} f=f$, as desired. 
(b) Theorem 3.14 implies that if $\bar{\partial}_{s} f=0$, then

$$
\bar{\partial}_{M}\left\{R\left([S]^{0,1} \wedge f\right)\right\}=[S]^{0,1} \wedge f
$$

which has support contained in $S$. Q.E.D.

We remark that the proof of part (a) of Theorem 5.4 would be much simpler if $R\left([S]^{0,1} \wedge f\right) \in C^{\infty}\left(\overline{M_{z_{0}}^{+}}\right) \cap C^{\infty}\left(\overline{M_{z_{0}}^{-}}\right)$(e.g. if $M$ is the sphere in $\left.\mathbf{C}^{n}\right)$.

Theorem 5.4 implies that a $\bar{\partial}_{S}$-closed form on $S$ is the continuous boundary value jump across $S-C_{S}$ of a $\bar{\partial}_{M}$-closed form on $M-S$. The next theorem is a local version of this phenomenon. The type of phenomenon in Theorems 5.4 and 5.5 is discussed in a more general context in [1]. What is new here is that we have an explicit kernel $R$ representing this jump, just as the Cauchy kernel in the complex plane represents any smooth function on a smooth curve as the boundary value jump of a holomorphic function.

5.5. Theorem. Suppose $z_{0} \in S-C_{S}$, and let $U$ be an open set in $S$ containing $z_{0}$. There exists an open set $\omega$ in $M$ containing $z_{0}$, with the following property. If $f \in \mathscr{D}_{S}^{p, q}\left(S-C_{S}\right)$, with $\bar{\partial}_{S} f \equiv 0$ on $U$, then there exists $F$ which is smooth on $\omega-S$, such that

(a) $\bar{\partial}_{M} F \equiv 0$ on $\omega-S$.

(b) $F \in C^{0}\left(\overline{M_{z}^{+}}\right) \cap C^{0}\left(\overline{M_{z}^{-}}\right)$for each $z \in \omega \cap S$.

(c) $F^{+}-F^{-}=f$ on $\omega \cap S$.

Proof. Suppose $f \in \mathscr{Q}_{S}^{p, q}\left(S-C_{S}\right)$ with $\bar{\partial}_{S} f \equiv 0$ on $U$. Theorem 5.4 implies $f=R^{+} f-R^{-} f$ on $S-C_{S}$. The trouble is that $R\left([S]^{0,1} \wedge f\right)$ is not $\bar{\partial}_{M}$ closed on $M-S$ near $z_{0}$. In fact, by Theorem 3.14, we have

$$
\bar{\partial}_{M}\left\{R\left([S]^{0,1} \wedge f\right)\right\}=R\left([S]^{0,1} \wedge \bar{\partial}_{S} f\right) \text { on } M-S
$$

Let $\omega$ be an open set in $M$ containing $z_{0}$ satisfying the hypothesis of Theorem 4.12 and such that $\omega \cap S \subset U$. Since $\bar{\partial}_{S} f \equiv 0$ on $\omega \cap S$ and since the singular set of the $R$ kernel is the diagonal, $R\left([S]^{0,1} \wedge \bar{\partial}_{S} f\right)$ is smooth near $\bar{\omega}$. Theorem 3.14 implies that $\bar{\partial}_{M}\left\{R\left([S]^{0,1} \wedge \bar{\partial}_{S} f\right)\right\} \equiv 0$ on $\omega$. Therefore, by Theorem 4.12, there exists $u \in \mathcal{E}_{M}^{p, q-1}(\omega)$ which solves the equation $\bar{\partial}_{M} u=R\left([S]^{0,1} \wedge \bar{\partial}_{S} f\right)$ on $\omega$. Let $F=R\left([S]^{0,1} \wedge f\right)-u$. In view of (5.6), $\bar{\partial}_{M} F \equiv 0$ on $\omega-S$. Theorem 5.2 implies part (b) of the theorem. Since $u$ is smooth on $\omega$, the boundary value jump of $u$ across $S \cap \omega$ is zero. Thus, $F^{+}-F^{-}=R^{+}(f)-R^{-}(f)=f$. Q.E.D.

ReMark. The formula for $F$ in Theorem 5.5 can be made explicit because the formula in Theorem 4.12 for $u$ is explicit.

5.3. The homotopy equation for $\bar{\partial}_{s}$. In this section, we shall derive a homotopy equation, from which the solution to $\bar{\partial}_{S}$ will drop out. Throughout this section, $S$ will satisfy the hypothesis of Theorem 5.3.

Recall that $\bar{\partial}_{M} E_{123}=E_{13}-E_{23}-R$ which holds off the singular sets of the kernels involved. If $f \in \mathscr{Q}_{S}^{p, q}\left(S-C_{S}\right)$, then we can apply the above formula to the 
current $[S]^{0,1} \wedge f$ and obtain

$$
\begin{aligned}
-\bar{\partial}_{M}\left\{E_{123}\left([S]^{0,1} \wedge f\right)\right\} & -E_{123}\left([S]^{0,1} \wedge \bar{\partial}_{S} f\right) \\
& =\left(E_{13}-E_{23}\right)\left([S]^{0,1} \wedge f\right)-R\left([S]^{0,1} \wedge f\right) .
\end{aligned}
$$

This equation holds on $M-S$, because all the forms involved are smooth on $M-S$. As before, we let $S_{t}=\{z \in M ; \operatorname{Re} h(z)=r(z)=t\}$. If we apply the orthogonal projection map $T_{S}$ to equation (5.7) and then restrict the equation to $S_{t}$, $t \neq 0$, then we obtain

$$
\begin{aligned}
R\left([S]^{0,1} \wedge f\right)_{T_{s}}= & \left(E_{13}-E_{23}\right)\left([S]^{0,1} \wedge f\right)_{T_{s}} \\
& +\bar{\partial}_{S_{1}}\left\{E_{123}\left([S]^{0,1} \wedge f\right)\right\}+E_{123}\left([S]^{0,1} \wedge \bar{\partial}_{S} f\right)_{T_{S}}
\end{aligned}
$$

which holds on $S_{t}$. Note that we have used that if $F$ is a smooth form on $M$, then $T_{S}\left\{\bar{\partial}_{M} F\right\}=\bar{\partial}_{S_{t}}\left\{F_{T_{s}}\right\}$ on $S_{t}$. (See Proposition 2.19.) Now $\bar{\partial}_{S_{t}}$ involves derivatives that are tangential to $S_{t}$ (i.e. vector fields which annihilate $r$ ). Therefore, by Theorems 5.2 and 5.3 we can take boundary values of the above equation from $M^{+}$and $M^{-}$(i.e. let $t \rightarrow 0^{+}$and $t \rightarrow 0^{-}$). We obtain

$$
R^{ \pm}(f)=\left(E_{13}^{ \pm}-E_{23}^{ \pm}\right)(f)+\bar{\partial}_{S}\left\{E_{123}^{ \pm}(f)\right\}+E_{123}^{ \pm}\left(\bar{\partial}_{S} f\right) \text {. }
$$

We set

$$
E=E_{123}^{+}-E_{123}^{-}, \quad Q=\left(E_{13}^{+}-E_{13}^{-}\right)+\left(E_{23}^{-}-E_{23}^{+}\right) .
$$

Theorem 5.3 implies that $E, Q$ map $\mathscr{D}_{S}^{*}\left(S-C_{S}\right)$ to $\mathcal{E}_{S}^{*}\left(S-C_{S}\right)$. If we use equation (5.8) and the jump formula for the $R$ kernel given in Theorem 5.4, then we obtain the following homotopy equation:

$$
f=Q f+\bar{\partial}_{S} E(f)+E\left(\bar{\partial}_{s} f\right) \text { on } S-C_{S} .
$$

Since $E_{13}(\zeta, z)$ has degree $n-2$ in $d \bar{\zeta}$ and $E_{23}(\zeta, z)$ has degree zero in $d \bar{\zeta}$, we get the following cases:

(a) If $f \in \mathscr{D}_{S}^{p, 0}\left(S-C_{S}\right)$, then $f=\left(E_{13}^{+}-E_{13}^{-}\right)(f)+E\left(\bar{\partial}_{s} f\right)$.

(b) If $f \in \mathscr{Q}_{S}^{p, q}\left(S-C_{S}\right)$ with $1 \leqslant q \leqslant n-3$, then $f=\bar{\partial}_{S}\{E(f)\}+E\left(\bar{\partial}_{S} f\right)$.

(c) If $f \in \mathscr{D}_{S}^{p, n-2}\left(S-C_{S}\right)$ satisfies condition $\mathcal{O}$ for $S$, then $f=\bar{\partial}_{S}\{E(f)\}$.

Note that $E_{23}(\zeta, z)$ is holomorphic in $\zeta$. Therefore if $f \in \mathscr{Q}_{S}^{p, n-2}\left(S-C_{S}\right)$ satisfies condition $\theta$ for $S$, then $E_{23}\left([S]^{0,1} \wedge f\right) \equiv 0$.

We also have the following $\mathcal{L}^{p}$ estimate on the operator $E$.

5.10. Lemma. Suppose $S \subset M$ satisfies the hypothesis of Theorem 5.3 and suppose $K_{1}$ and $K_{2}$ are compact sets contained in $S-C_{S}$. Then there is a constant $C_{K_{1} K_{2}}$ which depends only on the compact sets $K_{1}$ and $K_{2}$, such that if $f \in \mathscr{Q}_{S}^{p, q}\left(K_{1}\right)$, then

$$
\|E(f)\|_{\mathbb{P}\left(K_{2}\right)}<C_{K_{1} K_{2}}\|f\|_{\mathbb{P}\left(K_{1}\right)} \text {. }
$$

The proof of Lemma 5.10 will be given in $\$ 5.4$. Lemma 5.10 and the homotopy equation in (5.9), together, yield the main theorem of this chapter. 
5.11. THEOREM. Suppose $S \subset M=\partial D$ is a smooth oriented hypersurface in $M$ defined by $S=\{z \in M ; \operatorname{Re} h(z)=0\}$ where $h$ is holomorphic near $\bar{D}$. The operator $E: \mathscr{Q}_{S}^{*, q}\left(S-C_{S}\right) \rightarrow \mathcal{E}_{S}^{*, q-1}\left(S-C_{S}\right)$ also maps $\mathcal{L}_{p, \text { comp }}^{*}\left(S-C_{S}\right) \rightarrow \mathcal{L}_{p, \text { loc }}^{*}\left(S-C_{S}\right)$ continuously. If $f \in \mathscr{D}_{S}^{*, q}\left(S-C_{S}\right), 1 \leqslant q<n-3$, then

$$
f=\bar{\partial}_{s}\{E(f)\}+E\left(\bar{\partial}_{s} f\right) \text { on } S-C_{s} .
$$

Moreover, if $f \in \mathscr{Q}_{S}^{p, n-2}\left(S-C_{S}\right)$ satisfies condition $\mathcal{O}$ for $S$, then $f=\bar{\partial}_{S}\{E(f)\}$ on $S-C_{S}$.

So, for example, if $M$ is the unit sphere in $C^{n}$ and $S=\left\{z \in M ; \operatorname{Re} z_{1}=0\right\}$ is the equator, then we can solve the $\bar{\partial}_{S}$ equation on $S-\{( \pm i, 0, \ldots, 0)\}$.

5.4. Proofs of boundary value theorems. To prove Theorems 5.2 and 5.3, it will suffice to prove the following two theorems. Recall that $S=\{z \in M ; r(z)=0\}$ and $M^{+}=\{z \in M ; r(z)>0\} ; M^{-}=\{z \in M ; r(z)<0\}$. If $E$ is any one of our kernels, then we let

$$
E\left(\left[M^{ \pm}\right] \wedge f\right)(z)=\int_{\zeta \in M^{ \pm}} E(\zeta, z) \wedge f(\zeta)
$$

where it is understood that we only integrate the piece of $E(\zeta, z) \wedge f(\zeta)$ of type $(n, n-1)$ in $\zeta$.

5.12. TheOREM. Suppose $S$ is an arbitrary hypersurface in $M$ and $z_{0} \in S-C_{S}$. Let $\tilde{f} \in \mathcal{Q}_{M}^{p, q}(M)$ be a smooth form on $M$.

(a) If $\tilde{f}(\zeta)$ vanishes to order $j$ at $S$ near $z_{0}$, then $R\left(\left[M^{ \pm}\right] \wedge \tilde{f}\right) \in C^{j}\left(\overline{M_{z_{0}}^{\mp}}\right)$.

(b) The forms $L_{M}\left(\left[M^{ \pm}\right] \wedge \tilde{f}\right)$ and ${ }^{t} L_{M}\left(\left[M^{ \pm}\right] \wedge \tilde{f}\right)$ are in $C^{\infty}\left(\overline{M_{z_{0}}^{\mp}}\right)$.

5.13. ThEOREM. Suppose $S$ is a hypersurface in $M$ satisfying the hypothesis of Theorem 5.3, and let $z_{0} \in S-C_{S}$. Suppose $\tilde{f} \in \mathscr{Q}_{M}^{p, q}(M)$ and suppose $\tilde{f} \equiv 0$ near $C_{S}$.

(a) $E_{13}\left(\left[M^{ \pm}\right] \tilde{f}\right)$ and $E_{23}\left(\left[M^{ \pm}\right] \tilde{f}\right) \in C^{\infty}\left(\overline{M_{z_{0}}^{\bar{\tau}}}\right)$.

(b) If $f$ vanishes to order $j$ at $S$ near $z_{0}$, then $E_{123}\left(\left[M^{ \pm}\right] \tilde{f}\right) \in C^{j+1}\left(\overline{M_{z_{0}}^{\mp}}\right)$.

Proofs of Theorems 5.2 and 5.3 assuming Theorems 5.12 and 5.13. Suppose $f \in \mathscr{D}_{S}^{p, q}\left(S-C_{S}\right)$. Let $\tilde{f} \in \mathscr{D}_{M}^{p, q}(M)$ be any smooth extension of $f$ which vanishes near $C_{S}$. We note that $\bar{\partial}_{M}\left\{\left[M^{-}\right]\right\}=[S]^{0,1}$, and so

$$
\begin{aligned}
R\left([S]^{0,1} \wedge f\right) & =R\left(\bar{\partial}_{M}\left\{\left[M^{-}\right]\right\} \wedge \tilde{f}\right) \\
& =R\left(\bar{\partial}_{M}\left\{\left[M^{-}\right] \wedge \tilde{f}\right\}\right)-R\left(\left[M^{-}\right] \wedge \bar{\partial}_{M} \tilde{f}\right)
\end{aligned}
$$

which holds on $M^{+}$. Since $\bar{\partial}_{M} R=L_{M}-{ }^{t} L_{M}$, we have

$$
\begin{aligned}
R\left(\bar{\partial}_{M}\left\{\left[M^{-}\right] \wedge \tilde{f}\right\}\right) & =-\left(\bar{\partial}_{M} R\right)\left(\left[M^{-}\right] \wedge \tilde{f}\right)-\bar{\partial}_{M}\left\{R\left(\left[M^{-}\right] \wedge \tilde{f}\right)\right\} \\
& =\left({ }^{t} L_{M}-L_{M}\right)\left(\left[M^{-}\right] \tilde{f}\right)-\bar{\partial}_{M}\left\{R\left(\left[M^{-}\right] \tilde{f}\right)\right\}
\end{aligned}
$$


Therefore we have

$$
\begin{aligned}
R\left([S]^{0,1} \wedge f\right)= & \left({ }^{t} L_{M}-L_{M}\right)\left(\left[M^{-}\right] \tilde{f}\right)-\bar{\partial}_{M}\left\{R\left(\left[M^{-}\right] \tilde{f}\right)\right\} \\
& -R\left(\left[M^{-}\right] \bar{\partial}_{M} \tilde{f}\right) \text { on } M^{+} .
\end{aligned}
$$

Again, we let $S_{t}=\{z \in M ; r(z)=t\}$. We apply the tangential map $T_{S}$ to equation (5.14) and then restrict the equation to $S_{t}$ with $t>0$, we obtain

$$
\begin{aligned}
R\left([S]^{0,1} \wedge f\right)_{T_{s}}= & \left({ }^{t} L_{M}-L_{M}\right)\left(\left[M^{-}\right] \tilde{f}\right)_{T_{s}}-\bar{\partial}_{S_{t}}\left\{R\left(\left[M^{-}\right] \wedge f\right)_{T_{s}}\right\} \\
& -R\left(\left[M^{-}\right] \bar{\partial}_{M} \tilde{f}\right)_{T_{s}} \text { on } S_{t} .
\end{aligned}
$$

Since $\bar{\partial}_{S_{t}}$ only involves derivatives which are tangential to $S_{t}$, we can apply Theorem 5.12 to conclude that $R\left([S]^{0,1} \wedge f\right)_{T_{S}} \in C^{0}\left(\overline{M_{z_{0}}^{+}}\right)$, as desired.

(b) Suppose $f \in \mathscr{Q}_{S}^{p, 0}\left(S-C_{S}\right)$ with $\bar{\partial}_{S} f \equiv 0$ near $z_{0}$. Then we can choose the extension $\tilde{f}$ so that $\bar{\partial}_{M} \tilde{f}$ vanishes to infinite order at $S$ near $z_{0}$ (see [2]). From part (a) of Theorem 5.12, we see that $R\left(\left[M^{-}\right] \bar{\partial}_{M} \tilde{f}\right) \in C^{\infty}\left(\bar{M}_{z_{0}}^{+}\right)$. The second term on the right of (5.14) vanishes because of type considerations. $(R(\zeta, z)$ has degree less than $n-1$ in $d \bar{\zeta}$.) The first term lies in $C^{\infty}\left(\overline{M_{z_{0}}^{+}}\right)$by part (b) of Theorem 5.12. Therefore, $R\left([S]^{0,1} \wedge f\right) \in C^{\infty}\left(\overline{M_{z_{0}}^{+}}\right)$, as desired.

The above proof is the same if the + and - are reversed.

The proof of Theorem 5.3 is analogous to the proof of Theorem 5.2 and it will be left to the reader.

REMARKS. 1. Notice that $\bar{\partial}_{M}\left\{R\left(\left[M^{-}\right] \tilde{f}\right)\right\}$ involves derivatives which are normal to $S$. Thus, we do not know that this term is continuous up to $S$ from $M^{+}$. For this reason, we must apply the projection map $T_{S}$ to equation (5.14) before taking boundary values.

2. Proving Theorems 5.12 and 5.13 is easier than proving Theorems 5.2 and 5.3 directly. This is because the domain of integration is one dimension higher in Theorems 5.12 and 5.13 .

Proofs of TheOREMS 5.12 AND 5.13. Suppose $f \in \mathscr{D}_{M}^{n, n-1}(M)$ and vanishes near $C_{S}$. Suppose $h$ is a smooth function on $M \times M$ with $h(\zeta, z)=\theta\left(|\zeta-z|^{k}\right)$ where $k$ is a positive integer. If $f$ vanishes to order $j$ at $S$, then $f(\zeta)=\vartheta\left(r(\zeta)^{j}\right)$. By looking at the pieces of the kernels involved, we must examine

$$
(K f)(z)=\int_{\zeta \in M^{-}} \frac{f(\zeta) h(\zeta, z) r(\zeta)^{j}}{[u \cdot(\zeta-z)]^{p}\left[{ }^{t} u \cdot(\zeta-z)\right]^{q}[v \cdot(\zeta-z)]^{l}}
$$

where $p, q, l, j$ are nonnegative integers. It will be understood that if $l$ is positive, then $S$ must satisfy the hypothesis of Theorem 5.3. Otherwise, $S$ will be an arbitrary oriented hypersurface in $M$. With this understanding it is clear that $K f$ is smooth on $\mathrm{M}^{+}$. We must examine the behavior of $(K f)(z)$ as $z$ approaches $S$ from $M^{+}$. The case where + and - are reversed is similar. 
5.15. Lemma. Fix $z_{0} \in S-C_{s}$. $K(f)$ lies in the space $C^{s}\left(\overline{M_{z_{0}}^{+}}\right)$provided the following conditions are satisfied by $p, q, l, j, k, s$ and $n$. ( $n$ is the complex dimension of $\mathbf{C}^{n}$.)

(5.16) If $p$ and $q$ are positive, then $2(p+q)+l-k-j+s<2 n$.

(5.17) If $q=0$, then $p, l, s, j$ and $k$ can be arbitrary nonnegative integers. If $p=0$, then $q, l, s, j$ and $k$ can be arbitrary nonnegative integers.

To see that Lemma 5.15 implies Theorems 5.12 and 5.13, we check each kernel.

$R$ kernel. In this case, we have $p+q=n, l=0, k=1$. Condition (5.16) implies $s$ can be as large as $j$, but no larger.

$E_{123}$ kernel. In this case, we have $p+q=n-1, l=1$ and $k=1$. Condition (5.16) implies $s \leqslant j+1$.

Kernels $E_{13}, E_{23}, L$ or ${ }^{t} L$. It is easy to see that condition (5.17) applies to these kernels.

Proof of Lemma 5.15. It suffices to show that given $\zeta_{0}, z_{0} \in S-C_{S}$ with $\zeta_{0} \in B_{z_{0}}=\left\{\zeta \in M ; v\left(\zeta, z_{0}\right) \cdot\left(\zeta-z_{0}\right)=0\right\}$, there exists an open set $U_{1} \times U_{2} \subset$ $M \times M$ containing $\left(\zeta_{0}, z_{0}\right)$ such that if $f \in \mathscr{D}_{M}^{n, n-2}\left(U_{1}\right)$ then $K f$ has the required smoothness in $\overline{U_{2} \cap M^{+}}$. Then the lemma will follow from a partition of unity argument. When $l=0$, we only have to worry about the case when $\zeta_{0}=z_{0}$, because $u$ is a strong support function for $M$. So we must consider the following cases:

Case 1. $\zeta_{0}=z_{0}$ and $p, q \geqslant 1 ; 2(p+q)+l-k-j+s<2 n$.

Case 2. $\zeta_{0}=z_{0}$; either $p=0$ or $q=0$; if $q=0$ then $p, l, s, j$ and $k$ can be arbitrary nonnegative integers. If $p=0$, then $q, l, s, j$ and $k$ can be arbitrary nonnegative integers.

Case 3. $\zeta_{0} \neq z_{0} ; \zeta_{0} \in B_{z_{0}} ; l \geqslant 1$ and $p, q, l, j, s, k$ can be arbitrary nonnegative integers.

In all three cases, we must make a change of variables.

5.18. Lemma. For each $\left(\zeta_{0}, z_{0}\right) \in\left\{S-C_{S}\right\} \times\left\{S-C_{S}\right\}$ with $v\left(\zeta_{0}, z_{0}\right) \cdot\left(\zeta_{0}-z_{0}\right)$ $=0$ there exist $\eta>0$ and an open set $\tilde{U}_{1} \times \tilde{U}_{2} \subset \mathbf{C}^{n} \times \mathbf{C}^{n}$ containing $\left(\zeta_{0}, z_{0}\right)$ and $a$ map $\Psi: \tilde{U}_{1} \times \tilde{U}_{2} \rightarrow \mathbf{C}^{n}$ with the following properties.

(a) For each $z \in \tilde{U}_{2}$, the map $\Psi_{z}(\zeta)=\Psi(\zeta, z): \Psi_{z}^{-1}\{B(0, \eta)\} \rightarrow B(0, \eta)$ is a diffeomorphism, where $B(0, \eta)$ is the ball of radius $\eta$ about the origin. $\Psi_{z}^{-1}(w)$ is a smooth map in $z \in \tilde{U}_{2}$ and $w \in B(0, \eta)$.

(b) If we write $\Psi_{z}(\zeta)=\left(w_{1}(\zeta, z), \ldots, w_{n}(\zeta, z)\right)$ then $\operatorname{Re} w_{1}(\zeta, z)=\rho(\zeta)$, $\operatorname{Re} w_{2}(\zeta, z)=r(\zeta), \operatorname{Im} w_{2}(\zeta, z)=\operatorname{Im}\{v(\zeta, z) \cdot(\zeta-z)\}$.

(c) If in addition $\zeta_{0}=z_{0}$, we may choose $\Psi$ so that $\Psi_{z}(z)=0$ for $z \in \tilde{U}_{2} \cap S$, and $\operatorname{Im} w_{1}(\zeta, z)=\operatorname{Im}\{\hat{u} \cdot(\zeta-z)\}$.

Proof. The proof is similar to the proof of Lemma 4.22. In fact, since

$$
\operatorname{Re}\{v \cdot(\zeta-z)\}=r(\zeta)-r(z),
$$

we have $\Psi=\Psi_{1}-\left(0, r(z), 0^{\prime \prime}\right)$ where $\Psi_{1}$ is the map in Lemma 4.22. Q.E.D.

It is clear that we may require $\operatorname{Im} w_{1}=\operatorname{Im}\left\{{ }^{t} \hat{u} \cdot(\zeta-z)\right\}$ instead of $\operatorname{Im} w_{1}=$ $\operatorname{Im}\{u \cdot(\zeta-z)\}$ if we so desire. Such a diffeomorphism will be used if $p=0$. 
Cases 1 and 2. We assume $\zeta_{0}=z_{0} \in S-C_{S}$. In Cases 1 and 2 , we will be working near the diagonal, so we may replace $u$ by $\hat{u}$ in the definition of $K f$.

Throughout the rest of the proof of Lemma 5.15, we will use the following notation:

$$
\begin{aligned}
x_{j} & =\operatorname{Re} w_{j}, \quad 1 \leqslant j \leqslant n, \\
y_{j} & =\operatorname{Im} w_{j}, \quad 1 \leqslant j \leqslant n, \\
w^{\prime} & =\left(w_{2}, \ldots, w_{n}\right) \in \mathbf{C}^{n-1}, \\
w^{\prime \prime} & =\left(w_{3}, \ldots, w_{n}\right) \in \mathbf{C}^{n-2}, \\
w_{z} & =\left(y_{1}, w_{2}-r(z), w^{\prime \prime}\right) \in \mathbf{R}^{2 n-1} .
\end{aligned}
$$

$\mathbf{R}^{2 n-1}$ will have coordinates $\left(y_{1}, w^{\prime}\right)$ (i.e. $x_{1}=0$ ).

For each fixed $z \in M, \Psi_{z}\left(M^{-}\right)=\left\{w \in \mathbf{C}^{n} ; x_{1}=0\right.$ and $\left.x_{2}<0\right\}$. For shorthand, we write $\Psi_{z}\left(M^{-}\right)=\mathbf{R}_{-}^{2 n-1}$ which is independent of $z$.

Since $\Psi_{z}^{-1}\left(0, r(z), 0^{\prime \prime}\right)=z$ for $z \in M$, an easy Taylor series argument about the point $w_{0}=\left(0, r(z), 0^{\prime \prime}\right)$ shows that the estimate

$$
\left|\Psi_{z}^{-1}(w)-z\right| \approx\left|w_{z}\right|
$$

holds uniformly for $z \in M$ near $z_{0}$ and $w$ near 0 .

If $f(\zeta)$ has support near $z_{0}$, we may pull back the integral in $K f(z)$ via $\Psi_{z}^{-1}(\cdot)$ to obtain

$$
K f(z)=\int_{w \in \mathbf{R}_{-}^{2 n-1}} \frac{h_{1}(w, z) x_{2}^{j} f_{1}(w, z) d \lambda(w)}{u_{1}(w, z)^{p}{ }^{t} u_{1}(w, z)^{q}\left(w_{2}-r(z)\right)^{l}}
$$

where $d \lambda(w)$ is Lebesgue measure on $\mathbf{R}^{2 n-1}$ and where

$$
\begin{aligned}
h_{1}(w, z) & =h\left(\Psi_{z}^{-1}(w), z\right) \\
u_{1}(w, z) & =\hat{u}\left(\Psi_{z}^{-1}(w), z\right) \cdot\left(\Psi_{z}^{-1}(w)-z\right) \\
& =\operatorname{Re} u_{1}(w, z)+i y_{1} \\
{ }^{t} u_{1}(w, z) & ={ }^{t} \hat{u}\left(\Psi_{z}^{-1}(w), z\right) \cdot\left(\Psi_{z}^{1}(w)-z\right)
\end{aligned}
$$

and

$$
f_{1}(w, z) d \lambda=\Psi_{z}^{-1 *}\{f\}(w) .
$$

If $f$ has compact support near $z_{0}$, then $f_{1}(w, z)$ will have compact $w$-support near the origin.

Proof of CaSe 1. We will first assume $l \geqslant 1$. As we shall see, the proof of Case 1 when $l=0$ is similar and actually easier.

5.20. LeMMA. (a) $\left|w_{2}-r(z)\right| \approx\left|w_{2}\right|+r(z)$ for $z \in \overline{M^{+}}$and $x_{2}<0$.

(b) $\left|w_{z}\right|^{2} \approx|w|^{2}+r(z)^{2}$ for $z \in \overline{M^{+}}$and $x_{2} \leqslant 0$.

(c) $h_{1}(w, z)=\theta\left(\left|w_{z}\right|^{k}\right)$ for $z \in M$.

Given $z_{0} \in S$, there exist $\eta>0$ and an open set $U_{2}$ in $M$ containing $z_{0}$ such that the following estimates hold uniformly for $z \in U_{2}$ and $w \in \mathbf{R}_{-}^{2 n-1}$ with $|w| \leqslant \eta$.

(d) $u_{1}(w, z) \approx\left|w_{z}\right|^{2}+\left|y_{1}\right| ; \quad u_{1}(w, z) \approx\left|w_{z}\right|^{2}+\left|y_{1}\right|, \quad \operatorname{Re} u_{1}(w, z)=\theta\left(\left|w_{z}\right|^{2}\right) ;$ $\operatorname{Re}^{t} u_{1}(w, z)=\theta\left(\left|w_{z}\right|^{2}\right), u_{1}(w, z)-u_{1}(w, z)=\vartheta\left(\left|w_{z}\right|^{2}\right)$. 
(e) If $X_{z}$ is a vector field in $z$ which is tangential to $M$, then

$$
\begin{aligned}
& X_{z} u_{1}(w, z)=\mathcal{O}\left(\left|w_{z}\right|\right) ; \quad X_{z}{ }^{t} u_{1}(w, z)=\mathcal{O}\left(\left|w_{z}\right|\right), \\
& X_{z} h_{1}(w, z)= \begin{cases}\Theta\left(\left|w_{z}\right|^{k-1}\right) & \text { if } k \geqslant 1, \\
\Theta(1) & \text { if } k<1 .\end{cases}
\end{aligned}
$$

(f) If $X_{z}$ is a vector field in $z$ which is tangential to $S$, then

$$
\begin{gathered}
X_{z} u_{1}(w, z)= \\
X_{z}\left(\left|w_{z}\right|^{2}\right) ; \quad X_{z}{ }^{\prime} u_{1}(w, z)=\theta\left(\left|w_{z}\right|^{k}\right) .
\end{gathered}
$$

(g) For $2 \leqslant j \leqslant n$, we have

$$
\begin{aligned}
& \frac{\partial}{\partial y_{j}} u_{1}(w, z)=\vartheta\left(\left|w_{z}\right|\right) ; \frac{\partial}{\partial y_{j}}{ }^{t} u_{1}(w, z)=\vartheta\left(\left|w_{z}\right|\right), \\
& \frac{\partial}{\partial y_{j}} h_{1}(w, z)= \begin{cases}\Theta\left(\left|w_{z}\right|^{k-1}\right) & \text { if } k \geqslant 1, \\
\vartheta(1) & \text { if } k<1 .\end{cases}
\end{aligned}
$$

Proof. (a) We have $\left|w_{z}-r(z)\right| \approx\left|x_{2}-r(z)\right|+\left|y_{2}\right|$. If $x_{2} \leqslant 0$ and $r(z)>0$, then $\left|x_{2}-r(z)\right|=\left|x_{2}\right|+r(z)$. This establishes (a).

(b) We have $\left|w_{z}\right|^{2}=y_{1}^{2}+\left(x_{2}-r(z)\right)^{2}+y_{2}^{2}+\left|w^{\prime \prime}\right|^{2}$. Now $\left(x_{2}-r(z)\right)^{2}=x_{2}^{2}-$ $2 x_{2} r(z)+r(z)^{2}$. If $r(z) \geqslant 0$ and $x_{2} \leqslant 0$, then $\left(x_{2}-r(z)\right)^{2}>x_{2}^{2}+r(z)^{2}$. This establishes (b).

(c) Since $h(\zeta, z)=\theta\left(|\zeta-z|^{k}\right)$ by hypothesis, part (c) follows by letting $\zeta=$ $\Psi_{z}^{-1}(w)$ and using (5.19).

(d) Part (d) is proved exactly as part (b) in Lemma 4.19 is proved except that we use (5.19) instead of (4.18).

(e) and (f) We have $X_{z}\left\{u_{1}(w, z)\right\}=X_{z}\left\{\operatorname{Re} u_{1}(w, z)+i y_{1}\right\}=X_{z}\left\{\operatorname{Re} u_{1}(w, z)\right\}$. Since $\operatorname{Re} u_{1}(w, z)=\theta\left(\left|w_{z}\right|^{2}\right)$, we have that $\operatorname{Re} u_{1}(w, z)$ is a polynomial of degree two in the real components of $w_{z}=\left(y_{1}, w_{2}-r(z), w^{\prime \prime}\right)$ over the ring of smooth functions in $w$ and $z$. If $X_{z}$ is tangential to $S$, then $X_{z}\{r(z)\}=0$ and therefore $X_{z}\left\{\operatorname{Re} u_{1}(w, z)\right\}=\mathcal{O}\left(\left|w_{z}\right|^{2}\right)$. However, if $X_{z}$ is not tangential to $S$ then $X_{z}\{r(z)\} \neq$ 0 and so $X_{z}\left\{\operatorname{Re} u_{1}(w, z)\right\}=\theta\left(\left|w_{z}\right|\right)$.

Similarly, we have $X_{z} h_{1}(w, z)=\mathcal{O}\left(\left|w_{z}\right|^{k-1}\right)\left(\right.$ resp. $\left.\mathcal{O}\left(\left|w_{z}\right|^{k}\right)\right)$ if $X_{z}$ is not tangential to $S$ (resp. if $X_{z}$ is tangential to $S$ ). The estimate for $X_{z}\left\{{ }^{t} u_{1}(w, z)\right\}$ follows from the estimate on $X_{z}\left\{u_{1}(w, z)\right\}$ and because ${ }^{t} u_{1}(w, z)-u_{1}(w, z)=\theta\left(\left|w_{z}\right|^{2}\right)$.

(g) The estimates in (g) follow because $\partial / \partial y_{j}$ clearly lowers the degree of a polynomial in the components of $w_{z}$ by one. For example, if $j>2$ then

$$
\frac{\partial}{\partial y_{j}} u_{1}(w, z)=\frac{\partial}{\partial y_{j}}\left\{\operatorname{Re} u_{1}+i y_{1}\right\}=\frac{\partial}{\partial y_{j}} \vartheta\left(\left|w_{z}\right|^{2}\right)=\mathcal{\theta}\left(\left|w_{z}\right|\right) \text {. Q.E.D. }
$$

Remarks. 1. We emphasize that Lemma 5.20 only holds for $z \in M$ near $z_{0}$, because (5.19) holds only for $z \in M$ near $z_{0}$.

2. Parts (e) and (f) of Lemma 5.20 are the keys to understanding the difference between the boundary behavior of derivatives of $K f$ which are tangential to $S$ and derivatives of $K f$ which are not tangential to $S$. If $X_{z}$ is a vector field which is tangential to $S$, then, for example, the estimates on $\left|u_{1}\right|$ and $\left|X_{2} u_{1}\right|$ are the same, 
whereas if $X_{z}$ is not tangential to $S$, then the estimates on $\left|X_{z} u_{1}\right|$ are worse than the estimates on $\left|u_{1}\right|$ by a factor of $\Theta\left(\left|w_{z}\right|\right)$.

Now, let us return to $K f$. Choose $\eta>0$ according to Lemmas 5.20 and 5.18. Choose $U_{1} \times U_{2} \subset M \times M \cap\left\{\tilde{U}_{1} \times \tilde{U}_{2}\right\}$ containing $\left(\zeta_{0}, z_{0}\right)$, such that $U_{2}$ satisfies Lemma 5.20 and such that $\Psi\left\{\tilde{U}_{1} \times \tilde{U}_{2}\right\} \subset B(0, \eta)$. If $f \in \mathscr{D}_{M}^{n, n-1}\left(U_{1}\right)$ then $f_{1}(w, z)=f\left(\Psi_{z}^{-1}(w)\right)$ is smooth and for each $z \in U_{2}$, the $w$-support of $f_{1}(w, z)$ will be contained in $B(0, \eta)$.

We let $K_{1}(w, z)$ be the integrand occurring in $K f$, i.e.

$$
K_{1}(w, z)=\frac{h_{1}(w, z) x_{2}^{j} f_{1}(w, z)}{u_{1}^{p}{ }^{t} u_{1}^{q}\left(w_{2}-r(z)\right)^{l}} .
$$

We wish to get a single power of $w_{2}-r(z)$ in the denominator of $K_{1}(w, z)$. To do this, we write

$$
\left(w_{2}-r(z)\right)^{-l}=\frac{(i)^{l-1}}{(l-1) !}\left(\frac{\partial}{\partial y_{2}}\right)^{l-1}\left\{\left(w_{2}-r(z)\right)^{-1}\right\} .
$$

Since $f_{1}(w, z)$ has compact $w$-support, we may integrate by parts with $\partial / \partial y_{2}$ to obtain

$$
\begin{aligned}
(K f)(z) & =\int_{w \in \mathbf{R}_{-}^{2 n-1}} K_{1}(w, z) d \lambda(w) \\
& =\int_{w \in \mathbf{R}_{-}^{2 n-1}} \frac{(-i)^{l-1}}{(l-1) !}\left(\frac{\partial}{\partial y_{2}}\right)^{l-1}\left\{\frac{f_{1}(w, z) h_{1}(w, z)}{u_{1}^{p} u_{1}^{q}}\right\} \frac{x_{2}^{j} d \lambda(w)}{\left(w_{2}-r(z)\right)} \\
& =\int_{w \in \mathbf{R}_{-}^{2 n-1}} K_{2}(w, z) d \lambda(w)
\end{aligned}
$$

where $K_{2}$ is smooth with $w$-support contained in $B(0, \eta)$. Note, we could not have integrated by parts with $\partial / \partial x_{2}$ because we would have got boundary terms at $x_{2}=0$.

We need to estimate the integrand $K_{2}(w, z)$. From Lemma 5.20, we have

$$
\begin{aligned}
& \left|u_{1}(w, z)^{-p}\right| \lesssim\left(|w|^{2}+r(z)^{2}+\left|y_{1}\right|\right)^{-p} \\
& \left|u_{1}(w, z)^{-q}\right| \lesssim\left(|w|^{2}+r(z)^{2}+\left|y_{1}\right|\right)^{-q}
\end{aligned}
$$

We also have

$$
\begin{aligned}
\left|\frac{\partial}{\partial y_{2}}\left\{u_{1}(w, z)^{-p}\right\}\right| & =p\left|\left(\frac{\partial}{\partial y_{2}} u_{1}(w, z)\right)\left(u_{1}(w, z)\right)^{-(p+1)}\right| \\
& \lesssim \vartheta\left(\left|w_{z}\right|\right)\left(|w|^{2}+r(z)^{2}+\left|y_{1}\right|\right)^{-(p+1)} \\
& \lesssim\left(|w|^{2}+r(z)^{2}\right)^{1 / 2}\left(|w|^{2}+r(z)^{2}+\left|y_{1}\right|\right)^{-(p+1)} \\
& \lesssim\left(|w|^{2}+r(z)^{2}+\left|y_{1}\right|\right)^{-(p+1 / 2)} .
\end{aligned}
$$

Note that each time we differentiate $u_{1}^{-p}$ by $\partial / \partial y_{2}$, the estimate worsens by a factor of $\left(|w|^{2}+r(z)^{2}+\left|y_{1}\right|\right)^{-1 / 2}$. (Compare (5.22) with (5.21).) From these observations and from the rest of Lemma 5.20, we easily see that 


$$
\left|K_{2}(w, z)\right| \lesssim\left(|w|^{2}+r(z)^{2}+\left|y_{1}\right|\right)^{-\mu_{1}}\left(|w|^{2}+r(z)^{2}\right)^{\mu_{2}}\left(\left|w_{2}\right|+r(z)\right)^{-1}
$$

where $\mu_{1}=p+q+\frac{1}{2}(l-1), \mu_{2}=\frac{1}{2}(k+j)$. Therefore, we have

$$
\left|K_{2}(w, z)\right| \lesssim\left(|w|^{2}+r(z)^{2}+\left|y_{1}\right|\right)^{-v}\left(\left|w_{2}\right|+r(z)\right)^{-1}
$$

where $\nu=\mu_{1}-\mu_{2}=p+q+\frac{1}{2}(l-1-k-j)$. This estimate holds uniformly for $w \in \mathbf{R}_{-}^{2 n-1}$ with $|w| \leqslant \eta$ and $z \in U_{2} \cap \overline{M^{+}}$.

Now suppose $X_{z}$ is a vector field in $z$ on $M$. For $z \in M^{+} \cap U_{2}$, we have $X\{K f\}=\int_{w \in \mathbf{R}_{-}^{2 n-1}} X_{z} K_{2}(w, z) d \lambda(w)$. If $X_{z}$ is tangential to $S$ then $X(r) \equiv 0$ and so $X\left\{\left(w_{2}-r\right)^{-1}\right\}=0$. Therefore, $X_{z}$ must differentiate the rest of $K_{2}$ and Lemma 5.20 implies the estimates on $\left|X_{z}\left\{K_{2}(w, z)\right\}\right|$ and $\left|K_{2}(w, z)\right|$ are the same. For example,

$$
\begin{aligned}
\left|X\left\{u_{1}^{-p}\right\}\right| & =p\left|\left(X_{z} u_{1}(w, z)\right) u_{1}^{-(p+1)}\right| \\
& =\vartheta\left(\left|w_{z}\right|^{2}\right)\left(|w|^{2}+r(z)^{2}+\left|y_{1}\right|\right)^{-(p+1)} \\
& \lesssim\left(|w|^{2}+r(z)^{2}+\left|y_{1}\right|\right)^{-p}
\end{aligned}
$$

which is the same estimate satisfied by $\left|u_{1}^{-p}\right|$ (compare with (5.21)).

However, if $X_{z}$ is not tangential to $S$, then

$$
\begin{aligned}
\left|X\left\{u_{1}^{-p}\right\}\right| & =p\left|\left(X_{z} u_{1}(w, z)\right) u_{1}^{-(p+1)}\right| \\
& =\theta\left(\left|w_{z}\right|\right)\left(|w|^{2}+r(z)^{2}+\left|y_{1}\right|\right)^{-(p+1)} \\
& \lesssim\left(|w|^{2}+r(z)^{2}+\left|y_{1}\right|\right)^{-(p+1 / 2)} .
\end{aligned}
$$

So, we see that if $X$ is not tangential to $S$, then the estimate on $\left|X\left\{u_{1}^{-p}\right\}\right|$ is worse than the estimate on $\left|u_{1}^{-p}\right|$ by a factor of $\left(|w|^{2}+r(z)^{2}+\left|y_{1}\right|\right)^{-1 / 2}$.

Also, if $X_{z}$ is non-S-tangential, then $X\left\{\left(w_{2}-r\right)^{-1}\right\}=(X r)\left(w_{2}-r\right)^{-2}$, which is nonzero. So, we must integrate by parts as before with the vector field $\partial / \partial y_{2}$ to get a single power of $\left(w_{2}-r(z)\right)$ in the denominator. As we saw before, each time we integrate by parts with $\partial / \partial y_{2}$, the estimate on the integrand worsens by a factor of $\left(|w|^{2}+r(z)^{2}+\left|y_{1}\right|\right)^{-1 / 2}$.

In summary, suppose $X_{1}, \ldots, X_{N}$ are vector fields in $z$ on $M$ of which $s$ are not tangential to $S$. For $z \in M^{+} \cap U_{2}$, we have

$$
X_{1} \ldots X_{N}\{(K f)(z)\}=\int_{w \in \mathbf{R}_{-}^{2 n-1}} X_{1} \ldots X_{N}\left\{K_{2}(w, z)\right\} d \lambda(w) .
$$

After the appropriate integrations by parts, we have

$$
X_{1} \ldots X_{N}\{(K f)(z)\}=\int_{w \in \mathbf{R}_{-}^{2 n-1}} K_{3}(w, z) d \lambda(w)
$$

where $K_{3}$ is smooth and for each $z \in U_{2}$, the $w$-support of $K_{3}(w, z)$ is contained in $B(0, \eta)$. Moreover, we have

$$
\begin{aligned}
\left|K_{3}(w, z)\right| & \lesssim\left(|w|^{2}+r(z)^{2}+\left|y_{1}\right|\right)^{-\nu-s / 2}\left(\left|w_{2}\right|+r(z)\right)^{-1} \\
& \lesssim\left(\left|w^{\prime}\right|^{2}+\left|y_{1}\right|\right)^{-\nu-s / 2}\left|w_{2}\right|^{-1}
\end{aligned}
$$


which holds uniformly for $z \in \overline{M^{+}} \cap U_{2}$ and for $w \in \mathbf{R}_{-}^{2 n-1}$ with $|w| \leqslant \eta$. To prove that $X_{1} \ldots X_{N}\{K f\}$ has a continuous extension to $\overline{M^{+}} \cap U_{2}$, it suffices, by the dominated convergence theorem, to determine the values of $s$ which make the function $\left(\left|w^{\prime}\right|^{2}+\left|y_{1}\right|\right)^{-\nu-s / 2}\left|w_{2}\right|^{-1}$ locally integrable on $\mathbf{R}^{2 n-1}$. So suppose $\delta_{1}, \delta_{2}, \delta_{3}$ are fixed positive numbers. Consider

$$
I=\int_{\substack{w_{2} \in \mathbf{C} \\\left|w_{2}\right|<\delta_{1}}} \int_{\substack{w^{\prime \prime} \in \mathbf{R}^{2 n-4} \\\left|w^{\prime \prime}\right|<\delta_{2}}} \int_{0}^{\delta_{3}}\left(\left|w^{\prime \prime}\right|^{2}+\left|w_{2}\right|^{2}+\left|y_{1}\right|\right)^{-\nu-s / 2}\left|w_{2}\right|^{-1} d y_{1} d \lambda\left(w^{\prime \prime}\right) d \lambda\left(w_{2}\right)
$$

where $d \lambda\left(w^{\prime \prime}\right)$ and $d \lambda\left(w_{2}\right)$ are Lebesgue measures on $\mathbf{R}^{2 n-4}$ and $\mathbf{R}^{2}$, respectively. To determine $I$, we shall integrate $y_{1}$ first to obtain

$$
I=\theta(1)+\int_{\substack{w_{2} \in \mathbf{C} \\\left|w_{2}\right|<\delta_{1}}} \int_{\substack{w^{\prime \prime} \in \mathbf{R}^{2 n-4} \\\left|w^{\prime \prime}\right|<\delta_{2}}} \theta\left(\left|w^{\prime \prime}\right|^{2}+\left|w_{2}\right|^{2}\right)^{-\nu-s / 2+1}\left|w_{2}\right|^{-1} d \lambda\left(w^{\prime \prime}\right) d \lambda\left(w_{2}\right) .
$$

Next, we shall integrate $w^{\prime \prime} \in \mathbf{R}^{2 n-4}$. To do this, we use a lemma, which is proved using sophomore calculus.

5.24. Lemma. Suppose $\delta$ is a fixed positive real number and $a \in \mathbf{R}$. Let $m$ be an integer greater than one. Then

$$
\int_{\substack{|u|<\delta \\ u \in \mathbf{R}^{m}}}\left(|u|^{2}+a^{2}\right)^{-l} d \lambda(u)= \begin{cases}\vartheta(1)+\vartheta\left(a^{m-2 l}\right) & \text { if } l \neq m / 2, \\ \vartheta(\log a) & \text { if } l=m / 2 .\end{cases}
$$

We shall apply Lemma 5.24 with $a=\left|w_{2}\right|, l=\nu+s / 2-1$, and $m=2 n-4$, to obtain

$$
I=\theta(1)+\int_{\substack{w_{2} \in \mathrm{C} \\\left|w_{2}\right|<\delta_{1}}} \theta\left(\left|w_{2}\right|^{-(2 v-2 n+s+3)}\right) d \lambda\left(w_{2}\right) .
$$

Clearly, we see that $I<\infty$ if

$$
2 \nu-2 n+3+s<2 \text {. }
$$

Since $\nu=p+q+\frac{1}{2}(l-1-k-j),(5.25)$ will be satisfied if $2(p+q)+l-k-$ $j+s<2 n$, which is the condition assumed in Case 1 .

To summarize, if the condition in Case 1 on $p, q, l, j, k$ and $s$ is satisfied, then $K_{3}(z, w)$ is dominated uniformly for $z \in \overline{M^{+}} \cap U_{2}$ and $w \in \mathbf{R}_{-}^{2 n-1}$ with $|w|<\eta$ by a locally integrable function on $\mathbf{R}^{2 n-1}$. The dominated convergence theorem then implies that the function $X_{1} \ldots X_{N}\{K f\}(z)=\int_{w \in \mathbb{R}_{-}^{2 n-1}} K_{3}(w, z) d \lambda(w)$ has a continuous extension to $\overline{M^{+}} \cap U_{2}$, as desired. This proves Case 1 if $l>1$. If $l=0$, then the same proof applies except that we need not integrate by parts with $\partial / \partial y_{2}$. Note also that $S$ need not satisfy the hypothesis of Theorem 5.3.

Case 2. We will assume that $q=0$; the case when $p=0$ is similar. We will first assume $l \geqslant 1$. In this case, we have

$$
(K f)(z)=\int_{w \in \mathbf{R}_{-}^{2 n-1}} \frac{h_{1}(w, z) x_{2}^{j} f_{1}(w, z)}{u_{1}^{p}\left(w_{2}-r(z)\right)^{l}} d \lambda(w)
$$


where we have used the same notation as in Case 1. Now suppose $X_{1}, \ldots, X_{N}$ are vector fields in $z$ on $M$. For $z \in M^{+} \cap U_{2}$, we have

$$
X_{1} \ldots X_{N}\{K f(z)\}=\int_{w \in \mathbf{R}_{-}^{2 n-1}} X_{1} \ldots X_{N}\left\{\frac{h_{1}(w, z) x_{2}^{j} f_{1}(w, z)}{u_{1}(w, z)^{p}\left(w_{2}-r(z)\right)^{l}}\right\} d \lambda(w) .
$$

A typical term of $X_{1} \ldots X_{N}\{K f\}$ is

$$
K(z)=\int_{w \in \mathbf{R}_{-}^{2 n-1}} \frac{h_{2}(w, z) d \lambda(w)}{u_{1}(w, z)^{t_{1}}\left(w_{2}-r(z)\right)^{t_{2}}}
$$

where $h_{2}(w, z)$ is smooth with $w$-support contained in $B(0, \eta)$ and $t_{1}+t_{2}<p+l$ $+N$. Again, we wish to get a single power of $\left(w_{2}-r(z)\right)$ in the denominator. As in Case 1 , we integrate by parts with $\partial / \partial y_{2}$ to obtain

$$
K(z)=\frac{(-i)^{t_{2}-1}}{\left(t_{2}-1\right) !} \int_{w \in \mathbf{R}_{-}^{2 n-1}}\left(\frac{\partial}{\partial y_{2}}\right)^{t_{2}-1}\left\{\frac{h_{2}(w, z)}{u_{1}(w, z)^{t_{1}}}\right\}\left(w_{2}-r(z)\right)^{-1} d \lambda(w) .
$$

A typical term of $K(z)$ looks like

$$
K_{1}(z)=\int_{w \in \mathbf{R}_{-}^{2 n-1}} \frac{h_{3}(w, z)}{u_{1}(w, z)^{m}}\left(w_{2}-r(z)\right)^{-1} d \lambda(w)
$$

where $m$ is an integer with $m<t_{1}+t_{2}$ and $h_{3}(w, z)$ is smooth with $w$-support contained in $B(0, \eta)$. Now, we also want to get a single power of $u_{1}$ in the denominator. Actually, we will reduce the power one at a time by a technique used in the proof of Theorem 4.3. Let $g=(1 /(1-m))\left[\partial u_{1} / \partial y_{1}\right]^{-1}$, i.e.

$$
g(w, z)=\frac{1}{1-m}\left(\frac{\partial}{\partial y_{1}} \operatorname{Re} u_{1}(w, z)+i\right)^{-1} .
$$

Clearly, $g$ is smooth and $g \partial\left\{u_{1}^{1-m}\right\} / \partial y_{1}=u_{1}^{-m}$. This holds for $z \in M^{+} \cap U_{2}$ and $w \in \mathbf{R}_{-}^{2 n-1}$ with $|w|<\eta$. Integration by parts with $\partial / \partial y_{1}$ yields

$$
\begin{aligned}
K_{1}(z) & =\int_{w \in \mathbf{R}_{-}^{2 n-1}} u_{1}(w, z)^{1-m} \frac{\partial}{\partial y_{1}}\left\{h_{3} g\right\}\left(w_{2}-r(z)\right)^{-1} d \lambda(w) \\
& =\int_{w \in \mathbf{R}_{-}^{2 n-1}} u_{1}(w, z)^{1-m} h_{4}\left(w_{2}-r(z)\right)^{-1} d \lambda(w)
\end{aligned}
$$

where $h_{4}$ is smooth with $w$-support contained in $B(0, \eta)$. Iterating this procedure, we finally obtain for $z \in M^{+} \cap U_{2}$

$$
K_{1}(z)=\int_{w \in \mathbf{R}_{-}^{2 n-1}} u_{1}(w, z)^{-1} h_{5}(w, z)\left(w_{2}-r(z)\right)^{-1} d \lambda(w)
$$

where $h_{5}$ is smooth with $w$-support contained in $B(0, \eta)$. By Lemma 5.20 , the integrand is dominated by $\left(|w|^{2}+\left|y_{1}\right|\right)^{-1}\left|w_{2}\right|^{-1}$ uniformly for $z \in \overline{M^{+}} \cap U_{2}$ and $w \in \mathbf{R}_{-}^{2 n-1}$ with $|w|<\eta$. Since $\left(\left|w^{\prime}\right|+\left|y_{1}\right|\right)^{-1}\left|w_{2}\right|^{-1}$ is locally integrable on $\mathbf{R}^{2 n-1}$, the dominated convergence theorem implies that $K_{1}$ has a smooth extension to $\overline{M^{+}} \cap U_{2}$. Thus, $X_{1} \ldots X_{N}\{K f\}$ has a continuous extension to $\overline{M^{+}} \cap U_{2}$, as desired. 
As in Case 1, the proof of Case 2 when $l=0$ is easier. The above proof applies except the integration by parts with $\partial / \partial y_{2}$ is unnecessary. Again, if $l=0$, then we need not assume that $S$ satisfies the hypothesis of Theorem 5.3.

Case 3. Assume $\zeta_{0} \neq z_{0}$ and $v\left(\zeta_{0}, z_{0}\right) \cdot\left(\zeta_{0}-z_{0}\right)=0$ and $l \geqslant 1$. Since $\zeta_{0} \neq z_{0}$, we can require that $U_{1} \times U_{2} \cap \Delta=\varnothing$. Then, the function

$$
g(\zeta, z)=\frac{f(\zeta) h(\zeta, z) r(\zeta)^{j}}{u \cdot(\zeta-z)^{p}\left({ }^{t} u \cdot(\zeta-z)\right)^{q}}
$$

is smooth on $U_{1} \times U_{2}$. If $f \in \mathscr{D}_{M}^{n, n-1}\left(U_{1}\right)$ then the $\zeta$-support of $g(\zeta, z)$ is contained in $U_{1}$. Pulling the integral back via $\Psi_{z}^{-1}$, we obtain

$$
(K f)(z)=\int_{w \in \mathbf{R}_{-}^{2 n-1}} G(w, z)\left(w_{2}-r(z)\right)^{-l} d \lambda(w)
$$

where $G(w, z) d \lambda(w)=\Psi_{z}^{-1 *}\{g\}(w)$ is smooth and has compact $w$-support in $B(0, \eta)$. If $X_{1}, \ldots, X_{N}$ are vector fields in $z$ which are tangential to $M$ then

$$
X_{1} \ldots X_{N}\{K f\}(z)=\int_{w \in \mathbf{R}_{-}^{2 n-1}} X_{1} \ldots X_{N}\left\{G(w, z)\left(w_{2}-r(z)\right)^{-l}\right\} d \lambda(w)
$$

for $z \in M^{+} \cap U_{2}$. A typical term of $X_{1} \ldots X_{N}\{K f\}$ is

$$
K_{1}(z)=\int_{w \in \mathbf{R}_{-}^{2 n-1}} G_{1}(w, z)\left(w_{2}-r(z)\right)^{-t} d \lambda(w)
$$

where $G_{1}$ is smooth and has compact $w$-support in $B(0, \eta)$, and $t \leqslant l+N$. Using the vector field $\partial / \partial y_{2}$, we can integrate by parts $t-1$ times as in Cases 1 and 2, to obtain

$$
K_{1}(z)=\int_{w \in \mathbf{R}_{-}^{2 n-1}} G_{2}(w, z)\left(w_{2}-r(z)\right)^{-1} d \lambda(w)
$$

where $G_{2}$ is smooth, and for each $z \in U_{2} \cap M^{+}$, the w-support of $G_{2}$ is contained in $B(0, \eta)$.

For $w \in \mathbf{R}_{-}^{2 n-1}$ and $z \in \overline{M^{+}} \cap U_{2}$, the integrand in $K_{1}$ is dominated uniformly by $c\left|w_{2}\right|^{-1}$. Since $\left|w_{2}\right|^{-1}$ is locally integrable on $\mathbf{R}^{2 n-1}$, the dominated convergence theorem implies that $K_{1}$ has a continuous extension to $\overline{M^{+}} \cap U_{2}$, as desired. This proves Case 3, and concludes the proof of Lemma 5.15. Q.E.D.

Proof of Lemma 5.10. Before we prove Lemma 5.10, we need some preliminaries.

5.27. Lemma. Suppose $S$ is a hypersurface in $M$ satisfying the hypothesis of Theorem 5.3. Let $f \in \mathscr{Q}_{S}^{p, q}\left(S-C_{S}\right)$ and $g \in \mathscr{Q}_{S}^{n-p, n-q-1}\left(S-C_{S}\right)$. Then

$$
\left(E_{123}^{+}(f), g\right)_{S}=(-1)^{p+q+1}\left(f, E_{123}^{-}(g)\right)_{S} \text {. }
$$

Proof. Let $S_{\varepsilon}=\{z \in M ; r(z)=\varepsilon\}$. We have

$$
\begin{aligned}
\left(E_{123}^{+}(f), g\right)_{S} & =\lim _{\varepsilon \rightarrow 0^{-}}\left(E_{123}\left(\left[S_{\varepsilon}\right]^{0,1} \wedge f\right), g\right)_{S} \\
& =\lim _{\varepsilon \rightarrow 0^{-}}\left(E_{123}\left(\left[S_{\varepsilon}\right]^{0,2} \wedge f\right),[S]^{0,2} \wedge g\right)_{\mathbf{C}^{n}} \\
& =\lim _{\varepsilon \rightarrow 0^{-}}(-1)^{p+q+1}\left(\left[S_{\varepsilon}\right]^{0,2} \wedge f, E_{123}\left([S]^{0,2} \wedge g\right)\right)_{\mathbf{C}^{n}}
\end{aligned}
$$


where in the last equality, we have used that ${ }^{t} E_{123}=E_{123}$. Since $\varepsilon$ is approaching zero from negative values, we easily obtain $\left(E_{123}^{+}(f), g\right)_{S}=(-1)^{p+q+1}\left(f, E_{123}^{-}(g)\right)_{S}$ as desired. Q.E.D.

Lemma 5.27 implies that the operator $E=E_{123}^{+}-E_{123}^{-}$is selfadjoint, up to sign. Thus it will suffice to prove Lemma 5.10 for $p=\infty$. For then duality and Lemma 5.27 imply the result for $p=1$, i.e. we have

$$
\left|(E(f), g)_{S}\right|=\left|(f, E(g))_{S}\right| \leqslant C_{K}\|f\|_{\mathcal{E}^{\prime}(K)}\|g\|_{\mathfrak{E}^{\infty}(K)}
$$

for $f, g \in \mathscr{D}_{s}^{*}(K)$. Then, interpolation theory (see [18, Theorem 1.4.2]) implies Lemma 5.10 holds for all $1 \leqslant p \leqslant \infty$.

Thus, to prove Lemma 5.10, we must show that if $K_{1}, K_{2}$ are compact sets contained in $S-C_{S}$, then $\left|E_{123}^{+}(f)(z)-E_{123}^{-}(f)(z)\right|<C_{K_{1} K_{2}}\|f\|_{\mathfrak{L}^{\infty}\left(K_{1}\right)}$ holds for $z \in K_{2}$ and $f \in \mathscr{D}_{S}^{*}\left(K_{1}\right)$, where $C_{K_{1} K_{2}}$ is independent of $z \in K_{2}$, and $f$.

Let $Q(\zeta, z)=\left[\hat{u} \cdot(\zeta, z)^{p}\right]\left[{ }^{t} \hat{u} \cdot(\zeta, z)\right]^{q}$ where $p+q=n-1, p, q \geqslant 1$. A typical term of the kernel $E_{123}$ is $g(\zeta, z) /(Q(\zeta, z) v \cdot(\zeta-z))$ where $g(\zeta, z)=\theta(|\zeta-z|)$. By localizing the problem, we may assume that $g(\zeta, z)$ has $\zeta$-support contained near a fixed point $\zeta^{\prime} \in K_{1}$, and we will estimate $\left|\left(E_{123}^{+}-E_{123}^{-}\right)(f)(z)\right|$ for $z \in K_{2}$ near a fixed point $z^{\prime} \in K_{2}$.

5.28. Lemma. Given $z^{\prime} \in S-C_{S}$, there exists an open set $U$ in $S$ containing $z^{\prime}$ and there exists $a \delta>0$ and a smooth map $\phi: U \times[-\delta, \delta] \rightarrow M$ with $\phi(z, 0)=z$, $\operatorname{Im} \hat{u}(z, \phi(z, t)) \cdot(z-\phi(z, t))=0$, and $r(\phi(z, t))=t$, for $z \in U$ and $|t|<\delta$.

Proof. The desired map is $\phi(z, t)=\Psi_{z}^{-1}\left(0,0, t, 0,0^{\prime \prime}\right)$ where $\Psi_{z}(\cdot)$ is the diffeomorphism constructed in Lemma 5.18. Q.E.D.

For simplicity, we write $z_{t}=\phi(z, t)$. To prove Lemma 5.10, we must examine

$$
I^{r}=\int_{\zeta \in K}\left|\frac{g\left(\zeta, z_{r}\right)}{Q\left(\zeta, z_{r}\right) v \cdot\left(\zeta-z_{r}\right)}-\frac{g\left(\zeta, z_{-r}\right)}{Q\left(\zeta, z_{-r}\right) v \cdot\left(\zeta-z_{-r}\right)}\right| .
$$

The triangle inequality yields

$$
\begin{aligned}
I^{r} \leqslant & \int_{\zeta \in K}\left|\frac{g\left(\zeta, z_{r}\right)}{Q\left(\zeta, z_{r}\right)}\right|\left|\frac{1}{v \cdot\left(\zeta-z_{r}\right)}-\frac{1}{v \cdot\left(\zeta-z_{-r}\right)}\right| \\
& +\int_{\zeta \in K}\left|\frac{1}{v \cdot\left(\zeta-z_{-r}\right)}\right|\left|\frac{g\left(\zeta, z_{r}\right)}{Q\left(\zeta, z_{r}\right)}-\frac{g\left(\zeta, z_{-r}\right)}{Q\left(\zeta, z_{-r}\right)}\right| \\
= & I_{1}^{r}+I_{2}^{r} .
\end{aligned}
$$

We must show that given $\left(\zeta^{\prime}, z^{\prime}\right) \in K_{1} \times K_{2}$, then $\lim _{r \rightarrow 0^{+}}\left\{I_{1}^{r}+I_{2}^{r}\right\} \leqslant C_{K}$ holds uniformly for all $z \in K_{2}$ near $z^{\prime} \in K_{2}$ and where the $\zeta$-support of $g(\zeta, z)$ is contained near $\zeta^{\prime} \in K_{1}$.

Case 1. $\zeta^{\prime}=z^{\prime}$. We need to make a change of variables $w=\Psi_{z}(\zeta)$ described in Lemma 5.18. We can do this since $z^{\prime}$ is noncharacteristic. Note that $\Psi_{z}(S)=\mathbf{R}^{2 n-2}$ with coordinates $\left(y_{1}, y_{2}, w^{\prime \prime}\right)$, where $y_{j}=\operatorname{Im} w_{j}$ and $w^{\prime \prime}=\left(w_{3}, \ldots, w_{n}\right) \in \mathbf{C}^{n-2}$. Note also that on $\mathbf{R}^{2 n-2},\left|v \cdot\left(\Psi_{z}^{-1}(w)-z\right)\right| \gtrsim\left|y_{2}\right|+|r|$. So, if we pull back the 
integral in $I_{1}^{r}$, we obtain

$$
I_{1}^{r} \leqslant \int_{\substack{w \in \mathbf{R}^{2 n-2} \\|w|<\eta}}\left|\frac{g_{1}\left(w, z_{r}\right)}{Q_{1}\left(w, z_{r}\right)} \frac{2 r}{\left(y_{2}^{2}+r^{2}\right)}\right| d \lambda(w)
$$

where $d \lambda(w)$ is Lebesgue measure on $\mathbf{R}^{2 n-2}, g_{1}\left(w, z_{r}\right)=g\left(\Psi_{z}^{-1}(w), z_{r}\right), Q_{1}\left(w, z_{r}\right)=$ $Q\left(\Psi_{z}^{-1}(w), z_{r}\right)$, and $\eta$ is a fixed positive real number. Lemma 5.20 implies the following estimates hold uniformly for $z \in K_{2}$ near $z^{\prime}$ :

$$
\begin{aligned}
&\left|g_{1}\left(w, z_{r}\right)\right|=\theta(|w|+|r|), \\
&\left|\frac{1}{Q_{1}\left(w, z_{r}\right)}\right|<\frac{1}{\left(|w|^{2}+r^{2}+\left|y_{1}\right|\right)^{n-1}} .
\end{aligned}
$$

Since

$$
\int_{\left|y_{2}\right|<\eta} \frac{r}{y_{2}^{2}+r^{2}} d y_{2}=2 \tan ^{-1}\left(\frac{\eta}{r}\right)<\pi
$$

and since $\left(\left|w^{\prime \prime}\right|^{2}+\left|y_{1}\right|\right)^{3 / 2-n}$ is locally integrable on $\mathbf{R}^{2 n-3}$, we easily find that $I_{1}^{r} \leqslant C_{\eta}$ uniformly for $z \in K_{2}$ near $z^{\prime}$, and all $r$ with $|r| \leqslant \eta$.

We will show that $I_{2}^{r} \rightarrow 0$ as $|r| \rightarrow 0$. The triangle inequality yields

$$
\begin{aligned}
I_{2}^{r} \leqslant & \int_{\zeta \in K}\left|\frac{g\left(\zeta, z_{r}\right)-g\left(\zeta, z_{-r}\right)}{Q\left(\zeta, z_{r}\right)}\right|\left|\frac{1}{v \cdot\left(\zeta-z_{-r}\right)}\right| \\
& +\int_{\zeta \in K}\left|\frac{g\left(\zeta, z_{-r}\right)}{v \cdot\left(\zeta-z_{-r}\right)}\right|\left|\frac{1}{Q\left(\zeta, z_{r}\right)}-\frac{1}{Q\left(\zeta, z_{-r}\right)}\right| \\
& =' I_{2}^{r}+{ }^{\prime \prime} I_{2}^{r} .
\end{aligned}
$$

Pulling back the integral in ' $I_{2}^{r}$ via $\Psi_{z}^{-1}$, we have

$$
{ }^{\prime} I_{2}^{r} \leqslant \int_{\substack{w \in \mathbf{R}^{2 n-2} \\|w|<\eta}}\left|\frac{g_{1}\left(w, z_{r}\right)-g_{1}\left(w, z_{-r}\right)}{Q_{1}\left(w, z_{r}\right)}\right|\left|\frac{1}{\left|y_{2}\right|+|r|}\right| d \lambda(w) .
$$

Now $g_{1}\left(w, z_{r}\right)-g_{1}\left(w, z_{-r}\right)=\mathcal{O}\left(\left|z_{r}-z_{-r}\right|\right)=\mathcal{O}(|r|)$. Since

$$
\int_{\left|y_{2}\right|<\eta} \frac{|r| d y_{2}}{\left|y_{2}\right|+|r|}=\theta(|r| \log |r|)
$$

and since

$$
\int_{\substack{w \in \mathbf{R}^{2 n-3} \\|w|<\eta}}\left(\left|w^{\prime \prime}\right|^{2}+r^{2}+\left|y_{1}\right|\right)^{1-n} d \lambda(w)=\theta(\log |r|),
$$

we easily see that ' $I_{2}^{r} \leqslant \theta\left(|r|[\log |r|]^{2}\right)$. Thus ' $I_{2}^{r} \rightarrow 0$ as $|r| \rightarrow 0$.

Pulling back the integral in " $I_{2}^{r}$, we have

$$
" I_{2}^{r} \leqslant \int_{\substack{w \in \mathbf{R}^{2 n-2} \\|w|<\eta}}\left|\frac{g_{1}\left(w, z_{-r}\right)}{\left|y_{2}\right|+|r|}\right|\left|\frac{Q_{1}\left(w, z_{r}\right)-Q_{1}\left(w, z_{-r}\right)}{Q_{1}\left(w, z_{r}\right) Q_{1}\left(w, z_{-r}\right)}\right| d \lambda(w) .
$$


We claim that

$$
\begin{aligned}
\left|\frac{Q_{1}\left(w, z_{r}\right)-Q_{1}\left(w, z_{-r}\right)}{Q_{1}\left(w, z_{r}\right) Q_{1}\left(w, z_{-r}\right)}\right|= & \frac{\theta\left(\left|u_{1}\left(w, z_{r}\right)-u_{1}\left(w, z_{-r}\right)\right|\right)}{U^{n}} \\
& +\frac{\Theta\left(\left|u_{1}\left(w, z_{r}\right)-{ }^{t} u_{1}\left(w, z_{-r}\right)\right|\right)}{U^{n}}
\end{aligned}
$$

where $U^{n}$ is any expression of the form

$$
U^{n}(w, z)=\left[u_{1}\left(w, z_{r}\right)\right]^{j_{1}}\left[{ }^{t} u_{1}\left(w, z_{r}\right)\right]^{j_{2}}\left[u_{1}\left(w, z_{-r}\right)\right]^{j_{3}}\left[{ }^{t} u_{1}\left(w, z_{-r}\right)\right]^{j_{4}}
$$

with $j_{1}+j_{2}+j_{3}+j_{4}=n$. To prove (5.28), we induct on $\Rightarrow$ starting with $n=3$ $(\Rightarrow p=q=1)$. Let $u_{r}=u_{1}\left(w, z_{r}\right)$ and $Q_{r}=Q_{1}\left(w, z_{r}\right)$. Then, for $n=3$

$$
\begin{aligned}
\frac{Q_{r}-Q_{-r}}{Q_{r} Q_{-r}} & =\frac{\left(u_{r}-u_{-r}\right){ }^{t} u_{r}+u_{-r}\left({ }^{t} u_{r}-{ }^{t} u_{-r}\right)}{Q_{r} Q_{-r}} \\
& =\frac{\left(u_{r}-u_{-r}\right)}{u_{r} u_{-r}{ }^{t} u_{-r}}+\frac{\left({ }^{t} u_{r}-{ }^{t} u_{-r}\right)}{u_{r}{ }^{t} u_{r}{ }^{t} u_{-r}} .
\end{aligned}
$$

This proves the case when $n=3$.

To prove the induction step, we write

$$
\begin{aligned}
\frac{Q_{r}-Q_{-r}}{Q_{r} Q_{-r}} & =\frac{\left(u_{r}-u_{-r}\right) u_{r}^{p-1}{ }^{t} u_{r}^{q}+u_{-r}\left(u_{r}^{p-1}{ }^{t} u_{r}^{q}-u_{-r}^{p-1}{ }^{t} u_{-r}^{q}\right)}{Q_{r} Q_{-r}} \\
& =\frac{\left(u_{r}-u_{-r}\right)}{u_{r} u_{-r}^{p}{ }^{t} u_{-r}^{q}}+\frac{1}{u_{r}}\left[\frac{u_{r}^{p-1}{ }^{t} u_{r}^{q}-u_{-r}^{p-1}{ }^{t} u_{-r}^{q}}{u_{r}^{p-1}{ }^{t} u_{r}^{q} u_{-r}^{p-1}{ }^{t} u_{-r}^{q}}\right] .
\end{aligned}
$$

Since $p+q=n-1$, the first term is $\theta\left(u_{r}-u_{-r}\right) / U^{n}$. We may apply the induction hypothesis to the second term, and the claim follows.

5.31. LEMMA. The following estimates hold uniformly for $z \in K_{2}$ near $z^{\prime}$ and $w \in \mathbf{R}^{2 n-2}$ with $|w| \leqslant \eta$.

(a) $\left|g_{1}\left(w, z_{-r}\right)\right| \lesssim|w|+|r|$.

(b) $\left|U^{n}\left(w, z_{r}\right)\right| \gtrsim\left(|w|^{2}+r^{2}+\left|y_{1}\right|\right)^{-n}$.

(c)

$$
\begin{aligned}
& u_{1}\left(w, z_{r}\right)-u_{1}\left(w, z_{-r}\right)=\theta((|w|+|r|)|r|), \\
& { }^{t} u_{1}\left(w, z_{r}\right)-{ }^{t} u_{1}\left(w, z_{-r}\right)=\mathcal{O}((|w|+|r|)|r|) .
\end{aligned}
$$

Proof. The estimates in parts (a) and (b) follow from Lemma 5.20 and because $r\left(z_{r}\right)=r$. A Taylor series expansion of $\rho$ implies that $\operatorname{Re} \hat{u} \cdot(\zeta-z)=\theta\left(|\zeta-z|^{2}\right)$ and $\operatorname{Re}{ }^{t} \hat{u} \cdot(\zeta-z)=\theta\left(|\zeta-z|^{2}\right)$ for $(\zeta, z) \in M \times M$ near the diagonal. Therefore, by setting $\zeta=\Psi_{z}^{-1}(w)$, we easily obtain $\operatorname{Re} u_{1}\left(w, z_{r}\right)-\operatorname{Re} u_{1}\left(w, z_{-r}\right)=$ $\mathcal{\theta}((|w|+|r|)|r|)$ and $\operatorname{Re}{ }^{t} u_{1}\left(w, z_{r}\right)-\operatorname{Re}{ }^{t} u_{1}\left(w, z_{-r}\right)=\mathcal{\theta}((|w|+|r|)|r|)$.

Since $\operatorname{Im} \hat{u}\left(z, z_{r}\right) \cdot\left(z-z_{r}\right)=0$ (Lemma 5.28), we have $\operatorname{Im} u_{1}\left(w, z_{r}\right)$ $\operatorname{Im} u_{1}\left(w, z_{-r}\right)=\mathcal{O}((|w|+|r|)|r|)$. This in turn implies

$$
\begin{aligned}
\operatorname{Im}{ }^{t} u_{1}\left(w, z_{r}\right)-\operatorname{Im}{ }^{\prime} u_{1}\left(w, z_{-r}\right)= & {\left[\operatorname{Im}{ }^{t} u_{1}\left(w, z_{r}\right)-\operatorname{Im} u_{1}\left(w, z_{r}\right)\right] } \\
& -\left[\operatorname{Im}{ }^{\prime} u_{1}\left(w, z_{-r}\right)-\operatorname{Im} u_{1}\left(w, z_{-r}\right)\right] \\
& +\theta((|w|+|r|)|r|) .
\end{aligned}
$$


Now $u_{1}(w, z)-u_{1}(w, z)=\vartheta\left(\left|w_{z}\right|^{2}\right)$. Therefore, there exist smooth functions $a_{\alpha \beta}(w, z)$ such that

$$
\operatorname{Im} u_{1}\left(w, z_{r}\right)-\operatorname{Im} u_{1}\left(w, z_{r}\right)=\sum_{|\alpha|+|\beta|=2} a_{\alpha \beta}\left(w, z_{r}\right) w_{z_{r}}^{\alpha}\left(\bar{w}_{z_{r}}\right)^{\beta} .
$$

So from (5.32), we have

$$
\begin{aligned}
\operatorname{Im}{ }^{t} u_{1}\left(w, z_{r}\right)-\operatorname{Im}{ }^{t} u_{1}\left(w, z_{-r}\right)= & \sum_{|\alpha|+|\beta|=2} a_{\alpha \beta}\left(w, z_{r}\right) w_{z_{r}}^{\alpha}\left(\bar{w}_{z_{r}}\right)^{\beta} \\
& -a_{\alpha \beta}\left(w, z_{-r}\right) w_{z_{-r}}^{\alpha}\left(\bar{w}_{z_{-}}\right)^{\beta}+\theta((|w|+|r|)|r|) .
\end{aligned}
$$

Since $\left|w_{z_{r}}\right| \leqslant|w|+|r|$, an easy argument now shows that $\operatorname{Im}{ }^{t} u_{1}\left(w, z_{r}\right)-$ $\operatorname{Im}{ }^{t} u_{1}\left(w, z_{-r}\right)=\theta((|w|+r)|r|)$. This completes the proof of parts (b) and (c) of Lemma 5.30. Q.E.D.

In view of (5.29), (5.30) and the estimates in Lemma 5.31, we have

$$
\begin{aligned}
\left|g_{1}\left(w, z_{r}\right)\right| \frac{Q_{1}\left(w, z_{r}\right)-Q_{1}\left(w, z_{-r}\right)}{Q_{1}\left(w, z_{r}\right) Q_{1}\left(w, z_{-r}\right)} \mid & \lesssim \frac{\vartheta\left(|r|(|w|+|r|)^{2}\right)}{\left(|w|^{2}+r^{2}+\left|y_{1}\right|\right)^{n}} \\
& \lesssim \frac{\vartheta(|r|)}{\left(|w|^{2}+r^{2}+\left|y_{1}\right|\right)^{n-1}}
\end{aligned}
$$

Since $\int_{\left|y_{2}\right|<\eta} d y_{2} /\left|y_{2}\right|+|r|=\mathcal{O}(\log |r|)$ and since

$$
\int_{\substack{w \in \mathbf{R}^{2 n-3} \\|w|<\eta}}|r|\left(\left|w^{\prime \prime}\right|^{2}+r^{2}+\left|y_{1}\right|\right)^{1-n} d \lambda(w)=\vartheta(|r| \log |r|),
$$

we have " $I_{2}^{r}<C_{\eta}|r|[\log |r|]^{2}$. Thus, " $I_{2}^{r} \rightarrow 0$ as $|r| \rightarrow 0$, as desired.

Case 2. $\zeta^{\prime} \neq z^{\prime}$ and $v\left(\zeta^{\prime}, z^{\prime}\right) \cdot\left(\zeta^{\prime}-z^{\prime}\right)=0$. This case is easier. For $\zeta \neq z$, the function $g(\zeta, z) / Q(\zeta, z)$ is smooth. Therefore, if we pull back the integral in $I_{1}^{r}$ via $\Psi_{z}^{-1}$, then

$$
I_{1}^{r} \lesssim \int_{\left|y_{2}\right|<\eta} \frac{|r|}{y_{2}^{2}+r^{2}} d y_{2}<\pi
$$

which holds uniformly for $z \in K_{2}$ near $z^{\prime}$ and for $r$ with $|r| \leqslant \eta$. For $\zeta$ and $\zeta^{\prime}$ and $z$ near $z^{\prime}$, clearly, the following estimate holds:

$$
\left|\frac{g\left(\zeta, z_{r}\right)}{Q\left(\zeta, z_{r}\right)}-\frac{g\left(\zeta, z_{-r}\right)}{Q\left(\zeta-z_{-r}\right)}\right|=\vartheta(|r|)
$$

Therefore

$$
I_{2}^{r}<\int_{\left|y_{2}\right|<\eta} \frac{|r|}{\left|y_{2}\right|+|r|} d y_{2}=\theta(|r| \log |r|)
$$

Thus $I_{2}^{r} \rightarrow 0$ as $|r| \rightarrow 0$, as desired. This completes the proof of Lemma 5.10.

I would like to thank John Polking and Reese Harvey for their help during my graduate career at Rice University. 


\section{REFERENCES}

1. A. Andreotti, C. D. Hill, S. Kojasiewicz and B. MacKichan, Complexes of differential operators, Invent. Math. 35 (1976), 43-86.

2. A. Boggess, Plemelj jump formulas for the fundamental solution to $\bar{\partial}_{b}$ on the sphere, preprint.

3. J. Dadok and R. Harvey, The fundamental solution to $\square_{b}$ on the sphere in $\mathbf{C}^{n}$, Math. Ann. 244 (1979), 89-104.

4. G. B. Folland and J. J. Kohn, The Neumann problem for the Cauchy-Riemann complex, Ann. of Math. Studies, no. 75, Princeton Univ. Press, Princeton, N. J., 1972.

5. H. Grauert and I. Lieb, Das ramirezsche integral and die lösung der gleichung $\partial \vec{f}=\alpha$ im bereich der beschränktenformen, Complex Analysis (Proc. Conf. Rice University, Houston, Texas, 1969), Rice Univ. Studies 56, \#2, Rice University, Houston, 1970, pp. 29-50.

6. R. Harvey and J. Polking, Fundamental solutions in complex analysis. I, II, Duke Math. J. 46 (1979), 253-300; 301-340.

7. G. M. Henkin, Integral representations of functions holomorphic in strictly pseudo-convex domains and some applications, Mat. Sb. 78 (120) (1969), 611-632; English transl. in Math. USSR-Sb. 7 (1969), $597-616$.

8. Integral representations of functions holomorphic in strictly pseudo-convex domains and applications to the $\bar{\partial}$ problem, Mat. Sb. 82 (124) (1970), 300-308; English transl. in Math. USSR-Sb. 11 (1970), 273-281.

9. Solutions with estimates of the H. Lewy and Poincare-Lelong equations. The construction of functions of a Nevanlinna class with given zeros in a strictly pseudo-convex domain, Dokl. Akad. Nauk SSSR 224 (1975), 771-774; English transl. in Soviet Math. Dokl. 16 (1975), 1310-1314.

10. The H. Lewy equation and analysis of pseudo-convex manifolds, Uspehi Mat. Nauk 32 (1977), no. 3; English transl. in Russian Math. Surveys 32 (1977).

11. , H. Lewy's equation and analysis on a pseudo-convex manifolds. II, Mat. Sb. 102 (144) (1977), 71-108; English transl. in Math. USSR-Sb. 31 (1977), 63-94.

12. L. Hörmander, An introduction to complex analysis in several variables, Van Nostrand, Princeton, N. J., 1965.

13. L. R. Hunt, J. C. Polking and M. M. Strauss, Unique continuation for solutions to the induced Cauchy-Riemann equations, J. Differential Equations 23 (1977).

14. N. Øverlid, Integral representation formulas and $L^{p}$ estimates for the $\bar{\partial}$ equation, Math. Scand. 29 (1971), 137-160.

15. E. Ramirez de Arellano, En divisionsproblem und randintegraldarstellungen in der komplexen analysis, Math. Ann. 184 (1970), 172-187.

16. A. V. Romanov, A formula and estimates for the solutions of the tangential Cauchy-Riemann equations, Dokl. Akad. Nauk SSSR 220 (1975), 532-535; English transl. in Soviet Math. Dokl. 16 (1974), 124-128.

17. _ A formula and estimates for the solutions of the tangential Cauchy-Riemann equations, Mat. Sb. 99 (141) (1976), 58-83; English transl. in Math. USSR-Sb. 28 (1976).

18. E. M. Stein, Singular integrals and differentiability properties of functions, Princeton Univ. Press, Princeton, N. J., 1970.

19. R. O. Wells, Jr., Compact real submanifolds of a complex manifold with nondegenerate holomorphic tangent bundles, Math. Ann. 179 (1969), 123-129.

Department of Mathematics, University of Michigan, Ann Arbor, Michigan 48109 